\title{
Estimates on fractional higher derivatives of weak solutions for the Navier-Stokes equations
}

\author{
Kyudong Choi ${ }^{\mathrm{a}, *}$, Alexis F. Vasseur ${ }^{\mathrm{b}}$ \\ ${ }^{a}$ Department of Mathematics, University of Wisconsin-Madison, 480 Lincoln Dr., Madison, WI 53706, USA \\ ${ }^{\mathrm{b}}$ Department of Mathematics, University of Texas at Austin, 1 University Station C1200, Austin, TX 78712, USA \\ Received 21 November 2012; received in revised form 25 July 2013; accepted 6 August 2013
}

Available online 30 August 2013

\begin{abstract}
We study weak solutions of the 3D Navier-Stokes equations with $L^{2}$ initial data. We prove that $\nabla^{\alpha} u$ is locally integrable in space-time for any real $\alpha$ such that $1<\alpha<3$. Up to now, only the second derivative $\nabla^{2} u$ was known to be locally integrable by standard parabolic regularization. We also present sharp estimates of those quantities in weak- $L_{l o c}^{4 /(\alpha+1)}$. These estimates depend only on the $L^{2}$-norm of the initial data and on the domain of integration. Moreover, they are valid even for $\alpha \geqslant 3$ as long as $u$ is smooth. The proof uses a standard approximation of Navier-Stokes from Leray and blow-up techniques. The local study is based on De Giorgi techniques with a new pressure decomposition. To handle the non-locality of fractional Laplacians, Hardy space and Maximal functions are introduced.
\end{abstract}

๑) 2013 Elsevier Masson SAS. All rights reserved.

MSC: 76D05; 35Q30

Keywords: Navier-Stokes equations; Fluid mechanics; Blow-up techniques; Weak solutions; Higher derivatives; Fractional derivatives

\section{Introduction and the main result}

In this paper, any derivative signs $\left(\nabla, \Delta,(-\Delta)^{\alpha / 2}, D, \partial\right.$ and etc.) denote derivatives in the only space variable $x \in \mathbb{R}^{3}$ unless the time variable $t \in \mathbb{R}$ is clearly specified. We study the 3D Navier-Stokes equations

$$
\begin{aligned}
& \partial_{t} u+(u \cdot \nabla) u+\nabla P-\Delta u=0 \quad \text { and } \\
& \operatorname{div} u=0, \quad t \in(0, \infty), x \in \mathbb{R}^{3},
\end{aligned}
$$

with $L^{2}$ initial data

$$
u_{0} \in L^{2}\left(\mathbb{R}^{3}\right), \quad \operatorname{div} u_{0}=0 .
$$

The problem of global regularity of weak solutions for the 3D Navier-Stokes equations has a long history. Leray [27] 1930s and Hopf [22] 1950s proved the existence of a global-time weak solution for any given $L^{2}$ initial

\footnotetext{
* Corresponding author.

E-mail addresses: kchoi@math.wisc.edu (K. Choi), vasseur@math.utexas.edu (A.F. Vasseur).
} 
data. Such Leray-Hopf weak solutions are weak solutions $u$ of (1) lying in the functional class $L^{\infty}\left(0, \infty ; L^{2}\left(\mathbb{R}^{3}\right)\right) \cap$ $L^{2}\left(0, \infty ; H^{1}\left(\mathbb{R}^{3}\right)\right)$ and satisfying the following global energy inequality:

$$
\|u(t)\|_{L^{2}\left(\mathbb{R}^{3}\right)}^{2}+2\|\nabla u\|_{L^{2}\left(0, t ; L^{2}\left(\mathbb{R}^{3}\right)\right)}^{2} \leqslant\left\|u_{0}\right\|_{L^{2}\left(\mathbb{R}^{3}\right)}^{2} \quad \text { for a.e. } 0<t<\infty .
$$

Until now, regularity and uniqueness of such weak solutions are generally open.

Instead, many criteria which ensure regularity of weak solutions have been developed. Among them, the most famous one is Ladyženskaja-Prodi-Serrin Criteria [24,30,36], which says: if $u \in L^{p}\left((0, T) ; L^{q}\left(\mathbb{R}^{3}\right)\right)$ for some $p$ and $q$ satisfying $\frac{2}{p}+\frac{3}{q}=1$ and $p<\infty$, then it is regular. Recently, the limiting case $p=\infty$ was established in the paper of Escauriaza, Seregin and Šverák [16]. Similar criteria exist with various conditions on derivatives of velocity, vorticity, or pressure (see Beale, Kato and Majda [1], Beirão da Veiga [2] and Berselli and Galdi [4]). Also, many other conditions exist (e.g. see Cheskidov and Shvydkoy [10], Chan [9] and [5]).

On the other hand, many efforts have been devoted to the estimation of the size of the possible singular set where singularities may occur. This approach has been initiated by Scheffer [33]. Then, Caffarelli, Kohn and Nirenberg [6] improved the result and showed that possible singular sets have zero Hausdorff measure of one dimension for certain class of weak solutions (suitable weak solutions) satisfying the following additional inequality

$$
\partial_{t} \frac{|u|^{2}}{2}+\operatorname{div}\left(u \frac{|u|^{2}}{2}\right)+\operatorname{div}(u P)+|\nabla u|^{2}-\Delta \frac{|u|^{2}}{2} \leqslant 0
$$

in the sense of distribution. There are many other proofs of this fact (e.g. see Lin [28], [42] and Wolf [43]). Similar criteria for interior points with other quantities can be found in many places (e.g. see Struwe [40], Gustafson, Kang and Tsai [21], Seregin [35] and Chae, Kang and Lee [8]). Also, Robinson and Sadowski [31] and Kukavica [23] studied box-counting dimensions of singular sets.

In this paper, we consider space-time $L_{(t, x)}^{p}=L_{t}^{p} L_{x}^{p}$-estimates of higher derivatives for weak solutions assuming only $L^{2}$ initial data. The estimate $\nabla u \in L^{2}\left((0, \infty) \times \mathbb{R}^{3}\right)$ is obvious thanks to the energy inequality. A simple interpolation gives $u \in L^{10 / 3}$. For the second derivatives of weak solutions, a rough estimate $\nabla^{2} u \in L^{5 / 4}$ can be obtained by considering $(u \cdot \nabla) u$ as a source term from the standard parabolic regularization theory (see Ladyženskaja, Solonnikov and Ural'ceva [25]). With different ideas, Constantin [12] showed $\nabla^{2} u \in L^{\frac{4}{3}-\epsilon}$ for any small $\epsilon>0$ in periodic setting, and later Lions [29] improved it up to $\nabla^{2} u \in$ weak- $L^{\frac{4}{3}}$ (or $L^{\frac{4}{3}, \infty}$ ) by assuming that $\nabla u_{0}$ is lying in the space of all bounded measures in $\mathbb{R}^{3}$. They used natural structure of the equation with some interpolation technique. On the other hand, Foiaş, Guillopé and Temam [18] and Duff [15] obtained other kinds of estimates for higher derivatives of weak solutions while Giga and Sawada [19] and Dong and Du [14] covered mild solutions. For asymptotic behavior, we refer to Schonbek and Wiegner [34].

Recently in [41], it has been shown that, for any small $\epsilon>0$, any integer $d \geqslant 1$ and any smooth solution $u$ on $(0, T)$, there exist uniform bounds on $\nabla^{d} u$ in $L_{l o c}^{\frac{4}{d+1}-\epsilon}$, which depend only on the $L^{2}$-norm of the initial data once $\epsilon$, $d$ and the domain of integration are fixed. It can be considered as a natural extension of the result of Constantin [12] for higher derivatives. However, the method is very different. In [41], the proof uses the Galilean invariance of the equation and some regularity criterion of [42], which reproves the famous result of [6] by using a parabolic version of the De Giorgi method [13]. Note that this method gives full regularity to the critical Surface Quasi-Geostrophic equation in [7]. The exponent $p=\frac{4}{d+1}$ appears in a non-linear way from the following invariance of the Navier-Stokes scaling $u_{\lambda}(t, x)=\lambda u\left(\lambda^{2} t, \lambda x\right)$ :

$$
\left\|\nabla^{d} u_{\lambda}\right\|_{L^{p}}^{p}=\lambda^{-1}\left\|\nabla^{d} u\right\|_{L^{p}}^{p}
$$

In this paper, our main result improves the above result of [41] in the sense of the following three directions. First, we achieve the limiting case weak- $L^{\frac{4}{d+1}}$ (or $L^{\frac{4}{d+1}}, \infty$ ) as Lions [29] did for second derivatives. Second, we make similar bounds for fractional derivatives as well as classical derivatives. Last, we consider not only smooth solutions but also global-time weak solutions. These three improvements will give us that $\nabla^{3-\epsilon} u$, which is almost third derivatives of weak solutions, is locally integrable on $(0, \infty) \times \mathbb{R}^{3}$.

Our precise result is the following:

Theorem 1.1. There exist universal constants $C_{d, \alpha}$ which depend only on integer $d \geqslant 1$ and real $\alpha \in[0,2)$ with the following two properties (I) and (II): 
(I) Suppose that we have a smooth solution u of (1) on $(0, T) \times \mathbb{R}^{3}$ for some $0<T \leqslant \infty$ with some initial data (2). Then it satisfies

$$
\left\|(-\Delta)^{\frac{\alpha}{2}} \nabla^{d} u\right\|_{L^{p, \infty}\left(t_{0}, T ; L^{p, \infty}(K)\right)} \leqslant C_{d, \alpha}\left(\left\|u_{0}\right\|_{L^{2}\left(\mathbb{R}^{3}\right)}^{2}+\frac{|K|}{t_{0}}\right)^{\frac{1}{p}}
$$

for any $t_{0} \in(0, T)$, any integer $d \geqslant 1$, any $\alpha \in[0,2)$ and any bounded open subset $K$ of $\mathbb{R}^{3}$, where $p=\frac{4}{d+\alpha+1}$ and $|\cdot|=$ the Lebesgue measure in $\mathbb{R}^{3}$.

(II) For any initial data (2), we can construct a suitable weak solution $u$ of $(1)$ on $(0, \infty) \times \mathbb{R}^{3}$ such that $(-\Delta)^{\frac{\alpha}{2}} \nabla^{d} u$ is locally integrable in $(0, \infty) \times \mathbb{R}^{3}$ for $d=1,2$ and for $\alpha \in[0,2)$ with $(d+\alpha)<3$. Moreover, the estimate (5) holds with $T=\infty$ under the same setting of the above part (I) as long as $(d+\alpha)<3$.

Let us begin with some simple remarks.

Remark 1.1. For any suitable weak solution $u$, we can define $(-\Delta)^{\alpha / 2} \nabla^{d} u$ in the sense of distributions $\mathcal{D}^{\prime}$ for any integer $d \geqslant 0$ and for any real $\alpha \in[0,2)$ :

$$
\left\langle(-\Delta)^{\alpha / 2} \nabla^{d} u ; \psi\right\rangle>\mathcal{D}^{\prime}, \mathcal{D}=(-1)^{d} \int_{(0, \infty) \times \mathbb{R}^{3}} u \cdot(-\Delta)^{\alpha / 2} \nabla^{d} \psi d x d t
$$

for any test function $\psi \in \mathcal{D}=C_{c}^{\infty}\left((0, \infty) \times \mathbb{R}^{3}\right)$ where $(-\Delta)^{\alpha / 2}$ in the right-hand side is the traditional fractional Laplacian in $\mathbb{R}^{3}$ defined by the Fourier transform. Note that $(-\Delta)^{\alpha / 2} \nabla^{d} \psi$ lies in $L_{t}^{\infty} L_{x}^{2}$. Thus, this definition from (6) makes sense due to $u \in L_{t}^{\infty} L_{x}^{2}$. For the case $\alpha=0$, we define $(-\Delta)^{0}$ as the identity map. For more general extensions of this fractional Laplacian operator, we recommend Silvestre [37].

Remark 1.2. Since we impose only (2) to $u_{0}$, the estimate (5) is a (quantitative) regularization result to higher derivatives. Also, in the proof, we will see that $\left\|u_{0}\right\|_{L^{2}\left(\mathbb{R}^{3}\right)}^{2}$ in (5) can be relaxed to $\|\nabla u\|_{L^{2}\left((0, T) \times \mathbb{R}^{3}\right)}^{2}$. Thus it says that any (higher) derivatives can be controlled by having only $L^{2}$-estimate on the dissipation of energy.

Remark 1.3. The result of the part (I) for $\alpha=0$ extends the result of the previous paper [41] since for any $0<q<$ $p<\infty$ and any bounded subset $\Omega \subset \mathbb{R}^{n}$, we have

$$
\|f\|_{L^{q}(\Omega)} \leqslant C \cdot\|f\|_{L^{p, \infty}(\Omega)}
$$

where $C$ depends only on $p, q$, dimension $n$, and Lebesgue measure of $\Omega$ (e.g. see Grafakos [20]).

Remark 1.4. The "smoothness" assumption in the part (I) is about differentiability. For example, the result of the part (I) for $d \geqslant 1$ and $\alpha=0$ holds as long as $u$ is $d$-times differentiable. In addition, constants in (5) are independent of any possible blow-time $T$.

Remark 1.5. $p=4 /(d+\alpha+1)$ is a very interesting relation as mentioned before. Due to this $p$, the estimate (5) is a non-linear estimate while many other a priori estimates are linear. Estimates for $(d+\alpha)$ very close to 3, show that almost third derivatives of weak solutions are locally integrable. It would be very interesting to extend those results to values of $d<1$. The case $d=\alpha=0$, for instance, would imply that this weak solution $u$ lies in $L^{4, \infty}$ which is beyond the best known estimate $u \in L^{10 / 3}$ from $L^{2}$ initial data. This kind of extension, however, seems out of reach as now.

Remark 1.6. The exponent $p$ of the main estimate (5) can be obtained by dimensional analysis using the scaling invariance of the equation. As in [6], the scaling invariance is fundamental to our study. It allows us to guess the dependence of $p$ in terms of $(d+\alpha)$. Indeed, let $L_{x}$ be a typical length scale in space. Then the scaling $u_{\lambda}(t, x)=$ $\lambda u\left(\lambda^{2} t, \lambda x\right)$ implies that the typical time scale is $L_{t}=L_{x}^{2}$, any space derivative symbol $\nabla_{x}^{\alpha}$ has dimension $L_{x}^{-\alpha}$, and a solution $u$ has dimension $L_{x}^{-1}$. Then, taking $p$ th power on both sides of (5), the term $\left\|(-\Delta)^{\frac{\alpha}{2}} \nabla^{d} u\right\|_{L^{p, \infty}}^{p}$ on the left-hand side has dimension $L_{x}^{3+2-p(d+\alpha+1)}$ while both terms $\left\|u_{0}\right\|^{2}$ and $\frac{|K|}{t_{0}}$ on the right-hand side have dimension $L_{x}^{3-2}$. Thus $p$ should be $4 /(d+\alpha+1)$. 
Prior to presenting the main idea, we want to mention that [6] contains two different kinds of local regularity criteria. The first one is quantitative, and it says that if $\|u\|_{L^{3}(Q(1))}$ and $\|P\|_{L^{3 / 2}(Q(1))}$ are sufficiently small where $Q(r)$ is the parabolic cylinder $\left(-r^{2}, 0\right] \times B(r) \subset \mathbb{R} \times \mathbb{R}^{3}$, then $u$ is bounded by some universal constant in $Q(1 / 2)$. The second one says that $u$ is locally bounded near the origin if $\lim _{\sup _{r \rightarrow 0}} r^{-1}\|\nabla u\|_{L^{2}(Q(r))}^{2}$ is sufficiently small. So it is qualitative in the sense that the conclusion does not say that $u$ is bounded by a universal constant, but that sup $|u|$ is not infinite on some local neighbourhood. It also requires local estimates on an infinite number of zooms (via the limsup).

To explain the main idea and the scaling of the result, suppose that one could prove the following quantitative statement which requires only the smallness of $\|\nabla u\|_{L^{2}}$ :

If $\|\nabla u\|_{L^{2}(Q(1))} \leqslant \epsilon_{0}$ holds, for some absolute constant $\epsilon_{0}>0$, then $\left|\nabla^{d} u\right| \leqslant C_{d}$ in $Q(1 / 2)$ for $d \geqslant 1$ where $C_{d}$ depends only on $d$.

Then we could easily prove $\nabla^{d} u \in$ weak- $L_{l o c}^{4 /(d+1)}$ by using the contrapositive statement of the above one and the standard scaling together with Chebyshev's inequality. Indeed, applying the contrapositive statement on $u_{\lambda}(s, y)=$ $\lambda u\left(t+\lambda^{2} s, x+\lambda y\right)$ (and scaling back the result on $u$ ), we would get that for all $(t, x)$ such that $\left|\nabla^{d} u(t, x)\right| \geqslant \frac{C_{d}}{\lambda^{d+1}}$ with $t \geqslant \lambda^{2}>0$, we get $\frac{1}{\lambda^{5}} \int_{Q_{(t, x)}(\lambda)}|\nabla u(s, y)|^{2} d s d y \geqslant \epsilon_{0}^{2} \lambda^{-4}$ where $Q_{(t, x)}(r)=\left(t-r^{2}, t\right] \times B_{x}(r)$. Let $\lambda>0$. For $t \geqslant \lambda^{2}$ and $x \in \mathbb{R}^{3}$, denoting $F_{\lambda}(t, x)=\frac{1}{\lambda^{5}} \int_{Q_{(t, x)}(\lambda)}|\nabla u|^{2} d s d y$, by Chebyshev and Fubini, we get

$$
\begin{aligned}
& \mathcal{L}\left\{(t, x) \in\left(\lambda^{2}, \infty\right) \times \mathbb{R}^{3}:\left|\nabla^{d} u(t, x)\right| \geqslant \frac{C_{d}}{\lambda^{d+1}}\right\} \\
& \leqslant C \lambda^{4} \int_{\left(\lambda^{2}, \infty\right) \times \mathbb{R}^{3}} F_{\lambda} d t d x=C \lambda^{4} \int_{\left(\lambda^{2}, \infty\right) \times \mathbb{R}^{3}} \frac{1}{\lambda^{5}} \int_{Q(\lambda)}|\nabla u|^{2}(t+s, x+y) d s d y d t d x \\
& \leqslant C \lambda^{4}\left[\frac{1}{\lambda^{5}} \int_{Q(\lambda)} 1 d s d y\right] \int_{(0, \infty) \times \mathbb{R}^{3}}|\nabla u|^{2}(t, x) d t d x \leqslant C \lambda^{4}\left\|u_{0}\right\|_{L^{2}\left(\mathbb{R}^{3}\right)}^{2} .
\end{aligned}
$$

This would give the result. Note that the dependence of $p\left(=\frac{4}{d+1}\right)$ with respect to $d$ is unusual due to the non-linear estimate obtained through Chebyshev's inequality.

Unfortunately, the quantitative statement from above cannot be proven. This is due, in particular, to the long range effect of the pressure. Energy outside of the fixed region $Q(1)$ can have an effect (via the pressure) on the higher derivatives of $u$ in $Q(1 / 2)$. A different quantitative local regularity criterion has been proposed in [42], which showed that for any $p>1$, there exists $\epsilon_{p}>0$ such that

$$
\text { if }\|u\|_{L_{t}^{\infty} L_{x}^{2}(Q(1))}+\|\nabla u\|_{L_{t, x}^{2}(Q(1))}+\|P\|_{L_{t}^{p} L_{x}^{1}(Q(1))} \leqslant \epsilon_{p}, \quad \text { then }|u| \leqslant 1 \text { in } Q\left(\frac{1}{2}\right) .
$$

Recently, this criterion was used in [41] in order to obtain higher derivative estimates. The main proposition in [41] says that if both $\left\||\nabla u|^{2}+\left|\nabla^{2} P\right|\right\|_{L^{p}(Q(1))}$ and some other quantity about pressure (Maximal function of $\nabla^{2-\delta} P$ ) are small, then $u$ is bounded by 1 at the origin once $u$ has a mean zero property in space. We can observe that $\|\nabla u\|_{L^{2}(Q(1))}^{2}$ and $\left\|\nabla^{2} P\right\|_{L^{1}(Q(1))}$ have the same best scaling factor $\frac{1}{\lambda}$ (see (4)) among all well-known quantities which we can obtain from $L^{2}$ initial data. However, the other quantity about pressure has a slightly worse scaling factor than that of $\|\nabla u\|_{L^{2}}^{2}$. This is why the the limiting case $L^{\frac{4}{d+1}}, \infty$ could not be proved in [41].

To cover the limiting case $L^{\frac{4}{d+1}}, \infty$, we prove an equivalent estimate of (7) for $p=1$. This is achieved by introducing a new pressure decomposition (see Lemma 3.3). In fact, it can also be applied to solutions of some approximation scheme of the Navier-Stokes by adding one more assumption about the smallness of $\|\mathcal{M}(|\nabla u|)\|_{L^{2}}$ (see Proposition 2.1). We use the Leray regularization of the Navier-Stokes equation. This makes the drift velocity depend on the velocity via a convolution. It is then, not anymore local. We can still control it locally, via any zoom, thanks to the Maximal function. Then, the following will be shown in Section 5 by using the Galilean invariance and a blow-up technique with the standard scaling: 
Let $(u, P)$ be a smooth solution of the Navier-Stokes equations. We define $F:=\left(|\nabla u|^{2}+\left|\nabla^{2} P\right|\right)$ whose $L_{t, x}^{1}$-norm can be controlled by the $L^{2}$-norm of the initial data $u_{0}$ (see Lemma 5.1). Then for each point $(t, x)$ and for any $\epsilon$ such that $0<\epsilon^{2} \leqslant t$, there exist an incompressible flow $X(\cdot)$ with the following property:

If $\frac{1}{\epsilon} \iint_{Q_{(t, x)}(\epsilon)} F(s, y+X(s)) d y d s \leqslant \delta$, then $\left|\nabla^{d} u(t, x)\right| \leqslant C_{d} / \epsilon^{(d+1)}$ for $d \geqslant 1$.

The purpose of introducing the flow $X(\cdot)$ is to get a mean zero velocity. It enables us to avoid using any estimate of the velocity $u$ itself whose scaling is weaker than that of its derivative $\nabla u$. This kind of universal property comes from the local parabolic regularization effect of the viscosity term. However, if we do not control the main drift in a fixed region $Q(1)$, it could impair this process. The flow can be very fast in a fixed region $Q(1)$ so the fluid may pass through the region before having a chance to be locally regularized via the viscosity. Also, note that $F$ has the right scaling factor. As a consequence, thanks to the incompressibility of the flow $X(\cdot)$, we can prove $\nabla^{d} u \in L_{l o c}^{\frac{4}{d+1}, \infty}$ for classical derivatives ( $\alpha=0$ case) of smooth solutions of the Navier-Stokes equations.

The result for fractional derivatives $(0<\alpha<2$ case) is not obvious at all because there is no proper interpolation theorem for $L_{l o c}^{p, \infty}$ spaces. For example, due to the non-locality of the fractional Laplacian operator, the fact $\nabla^{2} u \in L_{l o c}^{\frac{4}{3}, \infty}$ with $\nabla^{3} u \in L_{l o c}^{1, \infty}$ does not imply the case of fractional derivatives even if we assume that $u$ is smooth. Moreover, even though we assume that $\nabla^{2} u \in L^{\frac{4}{3}}\left(\mathbb{R}^{3}\right)$ and $\nabla^{3} u \in L^{1}\left(\mathbb{R}^{3}\right)$ which we can NOT prove here, the standard interpolation theorem still requires $\nabla^{3} u \in L^{q}\left(\mathbb{R}^{3}\right.$ ) for some $q>1$ (we refer to Bergh and Löfström [3]).

To overcome the difficulty, we will use the Maximal function of $\nabla u$ which captures some behavior of $u$ in longrange distance. We will add some quantities depending on the Maximal functions of $\nabla u$ to $F$ (e.g. see the assumption of Proposition 2.2). This process should be done carefully because we want to add only functions whose scalings are correct. Unfortunately, the second derivatives of the pressure, which lie in the Hardy space $\mathcal{H} \subset L^{1}\left(\mathbb{R}^{3}\right)$ from Coifman, Lions, Meyer and Semmes [11], do not have an integrable Maximal function since the Maximal operator is not bounded on $L^{1}$. In order to handle non-local effects of the pressure, we will use some property of Hardy space, which says that some integrable functions play a similar role of the Maximal function (see (10)). This is the origin of the last term inside of the integral in (23) in Proposition 2.2.

Finally, the result $(I I)$ for weak solutions comes from a specific approximation of Navier-Stokes equations that Leray [27] used in order to construct a global-time weak solution: $\partial_{t} u_{n}+\left(\left(u_{n} * \phi_{(1 / n)}\right) \cdot \nabla\right) u_{n}+\nabla P_{n}-\Delta u_{n}=0$ and $\operatorname{div} u_{n}=0$ where $\phi$ is a fixed mollifier in $\mathbb{R}^{3}$, and $\phi_{(1 / n)}$ is defined by $\phi_{(1 / n)}(\cdot)=n^{3} \phi(n \cdot)$. The main advantage of adopting this approximation is that it has strong existence theory of global-time smooth solutions $u_{n}$ for each $n$, and it is well-known that there exists a suitable weak solution $u$ as a weak limit. In fact, for any integer $d \geqslant 1$ and for any $\alpha \in[0,2)$, we will obtain bounds for $u_{n}$ in the form of (5) with $T=\infty$, which is uniform in $n$. For the case $(d+\alpha)<3$, thanks to $p=4 /(d+\alpha+1)>1$, we can know that $(-\Delta)^{\frac{\alpha}{2}} \nabla^{d} u$ exists as a locally integrable function from weak-compactness of $L^{p}$ for $p>1$.

However, to prove (5) uniformly for the approximation is non-trivial because our proof is based on local study of De Giorgi-type argument while the approximation is not scaling-invariant with the standard Navier-Stokes scaling in the sense that $u * \phi_{(1 / n)}$ becomes $v * \phi_{1 /(n \epsilon)}$ (or see Remark 2.6). In other words, after the scaling, the convective velocity of the approximation scheme depends on the original velocity more non-locally than before. For example, once we fix $n$ and let $\epsilon$ go to zero, then we need information of the velocity $v$ in almost whole space to control the convective velocity $v * \phi_{1 /(n \epsilon)}$ in $Q(1)$.

It will be solved by separating its proof into two Lemmas 3.4 and 3.5. In the first lemma, the convective velocity is controlled by the Maximal function of $|\nabla u|$, which is not strong enough if the parameter $r:=\frac{1}{n \epsilon}$ is small. In the second lemma with small $r$, we use the fact that the convective velocity is not too different from the velocity itself for the first few steps. Then we can combine those two lemmas to get a uniform De Giorgi-type estimate $U_{k} \leqslant C^{k} U_{k-1}^{\beta}$ for some $\beta>1$ (see Section 3.4). The free parameter $r \in[0, \infty)$ has to be handled carefully also in the final bootstrapping arguments to get locally the control of higher derivatives (see Section 4.3).

The paper is organized as follows. In the next section, preliminaries with the main Propositions 2.1 and 2.2 will be introduced. Then we prove those Propositions 2.1 and 2.2 in Sections 3 and 4, respectively. Finally we will explain how Proposition 2.2 implies the part (II) of Theorem 1.1 for $\alpha=0$ and for $0<\alpha<2$ in Sections 5.2 and 5.3 respectively while the part (I) will be covered in Section 5.4. After that, Appendix A contains some missing proofs of technical lemmas. 


\section{Preliminaries, definitions and the main propositions}

We begin this section by fixing some notations and reminding some well-known results on analysis. After that, we will present definitions of two approximations and two main propositions. In this paper, any derivatives, convolutions and Maximal functions are with respect to the space variable $x \in \mathbb{R}^{3}$ unless the time variable is specified.

\subsection{Notations for general purpose}

We define $B(r)$, the ball in $\mathbb{R}^{3}$ centered at the origin with radius $r, Q(r)=\left(-r^{2}, 0\right] \times B(r)$, the parabolic cylinder in $\mathbb{R} \times \mathbb{R}^{3}$ and $B(x ; r)=B_{x}(r)$, the ball in $\mathbb{R}^{3}$ centered at $x$ with radius $r$.

Remark 2.1. In Section 3, we will introduce more notations for balls $B_{k}$ and cylinders $Q_{k}$ (see (24)), which decrease as $k$ increases. When using a De Giorgi-type argument, these notations are natural, and they will be used only in Sections 3.1, 3.2 and 3.3.

We fix $\phi \in C^{\infty}\left(\mathbb{R}^{3}\right)$ satisfying:

$$
\begin{aligned}
& \int_{\mathbb{R}^{3}} \phi(x) d x=1, \quad \operatorname{supp}(\phi) \subset B(1), \quad 0 \leqslant \phi \leqslant 1, \\
& \phi(x)=1 \quad \text { for }|x| \leqslant \frac{1}{2} \quad \text { and } \quad \phi \text { is radial. }
\end{aligned}
$$

For any real number $r>0$, we define the functions $\phi_{r} \in C^{\infty}\left(\mathbb{R}^{3}\right)$ by $\phi_{r}(x)=\frac{1}{r^{3}} \phi\left(\frac{x}{r}\right)$. When $r=0$, we define $\phi_{r}=$ $\phi_{0}=\delta_{0}$ as the Dirac-delta function. From Young's inequality for convolutions, we can observe

$$
\left\|f * \phi_{r}\right\|_{L^{p}(B(a))} \leqslant\|f\|_{L^{p}(B(a+r))}
$$

due to $\operatorname{supp}\left(\phi_{r}\right) \subset B(r)$ for any $p \in[1, \infty]$, for any $f \in L_{l o c}^{p}$ and for any $a, r>0$.

\section{2. $L^{p}$, weak- $L^{p}$ and Sobolev spaces $W^{n, p}$}

Let $K$ be an open subset $K$ of $\mathbb{R}^{n}$. For $0<p<\infty$, we denote $L^{p}(K)$ the usual space with (quasi) norm $\|f\|_{L^{p}(K)}=$ $\left(\int_{K}|f|^{p} d x\right)^{(1 / p)}$.

Also, for $0<p<\infty$, the weak- $L^{p}(K)$ space (or $L^{p, \infty}(K)$ ) is defined by

$$
L^{p, \infty}(K)=\left\{f \text { measurable in } K \subset \mathbb{R}^{d}: \sup _{\alpha>0}\left(\alpha^{p} \cdot|\{|f|>\alpha\} \cap K|\right)<\infty\right\}
$$

with (quasi) norm $\|f\|_{L^{p, \infty}(K)}=\sup _{\alpha>0}\left(\alpha \cdot|\{|f|>\alpha\} \cap K|^{1 / p}\right)$. From Chebyshev's inequality, we have $\|f\|_{L^{p, \infty}(K)} \leqslant$ $\|f\|_{L^{p}(K)}$ for any $0<p<\infty$. Also, for $0<q<p<\infty, L^{p, \infty}(K) \subset L^{q}(K)$ once $K$ is bounded (refer to Remark 1.3 in the beginning).

For any integer $n \geqslant 0$ and for any $p \in[1, \infty]$, we denote $W^{n, p}\left(\mathbb{R}^{3}\right)$ and $W^{n, p}(B(r))$ as the standard Sobolev spaces for the whole space $\mathbb{R}^{3}$ and for a ball $B(r)$ in $\mathbb{R}^{3}$, respectively.

\subsection{The Maximal function $\mathcal{M}$ and the Riesz transform $\mathcal{R}_{j}$}

The Maximal function $\mathcal{M}$ in $\mathbb{R}^{d}$ is defined by the following standard way:

$$
\mathcal{M}(f)(x)=\sup _{r>0} \frac{1}{|B(r)|} \int_{B(r)}|f(x+y)| d y .
$$

Also, we can express this Maximal operator as a supremum of convolutions: $\mathcal{M}(f)=C \sup _{\delta>0}\left(\chi_{\delta} *|f|\right)$ where $\chi=\mathbf{1}_{\{|x|<1\}}$ is the characteristic function of the unit ball, and $\chi_{\delta}(\cdot)=\left(1 / \delta^{3}\right) \chi(\cdot / \delta)$. Note that $\mathcal{M}$ is bounded from 
$L^{p}\left(\mathbb{R}^{d}\right)$ to $L^{p}\left(\mathbb{R}^{d}\right)$ for $p \in(1, \infty]$ and from $L^{1}\left(\mathbb{R}^{d}\right)$ to $L^{1, \infty}\left(\mathbb{R}^{d}\right)$. In this paper, we denote $\mathcal{M}$ and $\mathcal{M}^{(t)}$ as the Maximal functions in $\mathbb{R}^{3}$ and in $\mathbb{R}^{1}$, respectively.

For $1 \leqslant j \leqslant 3$, the Riesz Transform $\mathcal{R}_{j}$ in $\mathbb{R}^{3}$ is defined by:

$$
\widehat{\mathcal{R}_{j}(f)}(x)=i \frac{x_{j}}{|x|} \hat{f}(x) .
$$

The operator $\mathcal{R}_{j}$ is bounded in $L^{p}$ for $1<p<\infty$.

\subsection{The Hardy space $\mathcal{H}$}

The Hardy space $\mathcal{H}$ in $\mathbb{R}^{3}$ is defined by

$$
\mathcal{H}\left(\mathbb{R}^{3}\right)=\left\{f \in L^{1}\left(\mathbb{R}^{3}\right): \sup _{\delta>0}\left|\mathcal{P}_{\delta} * f\right| \in L^{1}\left(\mathbb{R}^{3}\right)\right\}
$$

where $\mathcal{P}=C\left(1+|x|^{2}\right)^{-2}$ is the Poisson kernel and $\mathcal{P}_{\delta}$ is defined by $\mathcal{P}_{\delta}(\cdot)=\delta^{-3} \mathcal{P}(\cdot / \delta)$. A norm of $\mathcal{H}$ is defined by $L^{1}$-norm of $\sup _{\delta>0}\left|\mathcal{P}_{\delta} * f\right|$. Thus $\mathcal{H}$ is a subspace of $L^{1}\left(\mathbb{R}^{3}\right)$ and $\|f\|_{L^{1}\left(\mathbb{R}^{3}\right)} \leqslant\|f\|_{\mathcal{H}\left(\mathbb{R}^{3}\right)}$ for any $f \in \mathcal{H}$. Moreover, the Riesz Transform is bounded from $\mathcal{H}$ to $\mathcal{H}$.

One of important applications of the Hardy space is the compensated compactness (see Coifman, Lions, Meyer and Semmes [11]). Especially, it says that if $E, B \in L^{2}\left(\mathbb{R}^{3}\right)$ and $\operatorname{curl} E=\operatorname{div} B=0$ in distribution, then $E \cdot B \in \mathcal{H}\left(\mathbb{R}^{3}\right)$ and we have

$$
\|E \cdot B\|_{\mathcal{H}\left(\mathbb{R}^{3}\right)} \leqslant C \cdot\|E\|_{L^{2}\left(\mathbb{R}^{3}\right)} \cdot\|B\|_{L^{2}\left(\mathbb{R}^{3}\right)}
$$

for some universal constant $C$. In order to obtain some regularity of second derivative of pressure, we can combine compensated compactness with boundedness of the Riesz transform in $\mathcal{H}\left(\mathbb{R}^{3}\right)$. For example, if $u$ is a weak solution of the Navier-Stokes (1), then a corresponding pressure $P$ satisfies

$$
\left\|\nabla^{2} P\right\|_{L^{1}\left(0, \infty ; \mathcal{H}\left(\mathbb{R}^{3}\right)\right)} \leqslant C \cdot\|\nabla u\|_{L^{2}\left(0, \infty ; L^{2}\left(\mathbb{R}^{3}\right)\right)}^{2}
$$

(see Lions [29] or Lemma 7 in [41]).

Note that if we replace the Poisson kernel $\mathcal{P}$ with any function $\mathcal{G} \in C^{\infty}\left(\mathbb{R}^{3}\right)$ with compact support, then we have a constant $C$ depending only on $\mathcal{G}$ such that

$$
\left\|\sup _{\delta>0}\left|\mathcal{G}_{\delta} * f\right|\right\|_{L^{1}\left(\mathbb{R}^{3}\right)} \leqslant C\left\|\sup _{\delta>0}\left|\mathcal{P}_{\delta} * f\right|\right\|_{L^{1}\left(\mathbb{R}^{3}\right)}=C\|f\|_{\mathcal{H}\left(\mathbb{R}^{3}\right)}
$$

where $\mathcal{G}_{\delta}(\cdot)=\mathcal{G}(\cdot / \delta) / \delta^{3}$ (see Fefferman and Stein [17] or see Stein [39], Grafakos [20] for modern texts). Even though the Maximal function $\sup _{\delta>0}\left(\chi_{\delta} *|f|\right)$ of any non-trivial Hardy space function $f$ is not integrable, there exist integrable functions $\left(\sup _{\delta>0}\left|\mathcal{G}_{\delta} * f\right|\right)$, which can capture locally some non-local feature of the function $f$ in a similar way Maximal functions do. However, (10) is slightly weaker than the Maximal function, since it controls only mean values of non-local quantities (not the absolute value). This weakness is the reason that we introduce certain definitions of $\zeta$ and $h^{\alpha}$ in the following.

\subsection{Notations associated to the fractional derivatives $(-\Delta)^{\alpha / 2}$}

The following two definitions of $\zeta$ and $h^{\alpha}$ will be used only in the proof concerning fractional derivatives. We define $\zeta$ by $\zeta(x)=\phi\left(\frac{x}{2}\right)-\phi(x)$. Then we have

$$
\begin{aligned}
& \zeta \in C^{\infty}\left(\mathbb{R}^{3}\right), \quad \operatorname{supp}(\zeta) \subset B(2), \quad \zeta(x)=0 \quad \text { for }|x| \leqslant \frac{1}{2} \quad \text { and } \\
& \sum_{j=k}^{\infty} \zeta\left(\frac{x}{2^{j}}\right)=1 \quad \text { for }|x| \geqslant 2^{k} \text { for any integer } k .
\end{aligned}
$$

In addition, we define the function $h^{\alpha}$ for $\alpha>0$ by $h^{\alpha}(x)=\zeta(x) /|x|^{3+\alpha}$. Also we define $\left(h^{\alpha}\right)_{\delta}$ and $\left(\nabla^{d} h^{\alpha}\right)_{\delta}$ by $\left(h^{\alpha}\right)_{\delta}(x)=\delta^{-3} h^{\alpha}(x / \delta)$ and $\left(\nabla^{d} h^{\alpha}\right)_{\delta}(x)=\delta^{-3}\left(\nabla^{d} h^{\alpha}\right)(x / \delta)$ for $\delta>0$ and for positive integer $d$, respectively. Then they satisfy 


$$
\begin{aligned}
& \left(h^{\alpha}\right)_{\delta} \in C^{\infty}\left(\mathbb{R}^{3}\right), \quad \operatorname{supp}\left(\left(h^{\alpha}\right)_{\delta}\right) \subset B(2 \delta)-B(\delta / 2) \quad \text { and } \\
& \frac{1}{|x|^{3+\alpha}} \cdot \zeta\left(\frac{x}{2^{j}}\right)=\frac{1}{\left(2^{j}\right)^{\alpha}} \cdot\left(h^{\alpha}\right)_{2^{j}}(x) \quad \text { for any integer } j .
\end{aligned}
$$

\subsection{The definition of the fractional Laplacian $(-\Delta)^{\alpha / 2}$}

For $-3<\alpha \leqslant 2$ and for $f \in \mathcal{S}\left(\mathbb{R}^{3}\right)$ (the Schwartz space), $(-\Delta)^{\frac{\alpha}{2}} f$ is defined by the Fourier transform:

$$
\widehat{(-\Delta)^{\frac{\alpha}{2}}} f(\xi)=|\xi|^{\alpha} \hat{f}(\xi) \text {. }
$$

Note that $(-\Delta)^{0}=I d$. Especially, for $\alpha \in(0,2)$, the fractional Laplacian can also be defined by the singular integral for any $f \in \mathcal{S}$ :

$$
(-\Delta)^{\frac{\alpha}{2}} f(x)=C_{\alpha} \cdot P . V . \int_{\mathbb{R}^{3}} \frac{f(x)-f(y)}{|x-y|^{3+\alpha}} d y .
$$

We introduce two approximations to the Navier-Stokes. The first one (Problem I- $n$ ) is the approximation, which Leray [27] used, while the second one (Problem II- $r$ ) will be used in our local study after we apply some certain scaling to (Problem I- $n$ ).

\subsection{Definition of (Problem I-n): the first approximation to Navier-Stokes}

Definition 2.1. Let $n \geqslant 1$ be either an integer or the infinity $\infty$, and let $0<T \leqslant \infty$. Suppose that $u_{0}$ satisfy (2). We say that $(u, P) \in\left[C^{\infty}\left((0, T) \times \mathbb{R}^{3}\right)\right]^{2}$ is a solution of (Problem I- $\left.n\right)$ on $(0, T)$ for the data $u_{0}$ if it satisfies

$$
\begin{aligned}
& \partial_{t} u+\left(\left(u * \phi_{\frac{1}{n}}\right) \cdot \nabla\right) u+\nabla P-\Delta u=0, \\
& \operatorname{div} u=0, \quad t \in(0, T), \quad x \in \mathbb{R}^{3},
\end{aligned}
$$

and

$$
u(t) \rightarrow u_{0} * \phi_{\frac{1}{n}} \quad \text { in } L^{2} \text {-sense as } t \rightarrow 0
$$

Remark 2.2. When $n=\infty$, (15) is the Navier-Stokes on $(0, T) \times \mathbb{R}^{3}$ with the initial value $u_{0}$.

Remark 2.3. If $n$ is not the infinity but a positive integer, then for any given $u_{0}$ of (2), we have existence and uniqueness theory of (Problem I- $n$ ) on $(0, \infty)$ with the energy equality

$$
\|u(t)\|_{L^{2}\left(\mathbb{R}^{3}\right)}^{2}+2\|\nabla u\|_{L^{2}\left(0, t ; L^{2}\left(\mathbb{R}^{3}\right)\right)}^{2}=\left\|u_{0} * \phi_{\frac{1}{n}}\right\|_{L^{2}\left(\mathbb{R}^{3}\right)}^{2},
$$

for any $t<\infty$. It is well-known that we can extract a sub-sequence which converges to a suitable weak solution $u$ of (1) and (3) with the initial data $u_{0}$ of (2) (see Leray [27], or see Lions [29], Lemarié-Rieusset [26] for modern texts).

Remark 2.4. As mentioned in the introduction section, we can observe that, for $n<\infty$, this notion (Problem I- $n$ ) is not invariant under the standard Navier-Stokes scaling $u(t, x) \rightarrow \epsilon u\left(\epsilon^{2} t, \epsilon x\right)$ due to the convective velocity $\left(u * \phi_{1 / n}\right)$.

\subsection{Definition of (Problem II-r): the second approximation to Navier-Stokes}

Definition 2.2. Let $0 \leqslant r<\infty$ be real. We say that $(u, P) \in\left[C^{\infty}\left((-4,0) \times \mathbb{R}^{3}\right)\right]^{2}$ is a solution of (Problem II- $\left.r\right)$ if it satisfies

$$
\begin{aligned}
& \partial_{t} u+(w \cdot \nabla) u+\nabla P-\Delta u=0, \\
& \operatorname{div} u=0, \quad t \in(-4,0), \quad x \in \mathbb{R}^{3},
\end{aligned}
$$

where $w$ is the difference of two functions: 


$$
w(t, x)=w^{\prime}(t, x)-w^{\prime \prime}(t), \quad t \in(-4,0), x \in \mathbb{R}^{3},
$$

which are defined by $u$ in the following way:

$$
w^{\prime}(t, x)=\left(u * \phi_{r}\right)(t, x) \quad \text { and } \quad w^{\prime \prime}(t)=\int_{\mathbb{R}^{3}} \phi(y)\left(u * \phi_{r}\right)(t, y) d y .
$$

Remark 2.5. This notion of (Problem II- $r$ ) gives us the mean zero property for the convective velocity $w$ : $\int_{\mathbb{R}^{3}} \phi(x) w(t, x) d x=0$ on $(-4,0)$. Also this $w$ is divergent free from the definition. Moreover, by multiplying $u$ to (18), we have

$$
\partial_{t} \frac{|u|^{2}}{2}+\operatorname{div}\left(w \frac{|u|^{2}}{2}\right)+\operatorname{div}(u P)+|\nabla u|^{2}-\Delta \frac{|u|^{2}}{2}=0
$$

in classical sense because our definition assumes $(u, P) \in C^{\infty}$.

Remark 2.6. We will introduce some specially designed $\epsilon$-scaling which will be a bridge between (Problem I- $n$ ) and (Problem II- $r$ ) (it can be found in (96) in Section 5). This scaling is based on the Galilean invariance in order to obtain the mean zero property for the velocity $u: \int_{\mathbb{R}^{3}} \phi(x) u(t, x) d x=0$ on $(-4,0)$. Moreover, this $\epsilon$-scaling applied to solutions of (Problem I- $n$ ), provides a solution to (Problem II- $\frac{1}{n \epsilon}$ ) (it can be found in (97)). We need a local result which is independent of both $\epsilon$ and $n$. In other words, we have to consider the free parameter $r:=\frac{1}{n \epsilon} \in[0, \infty)$.

Remark 2.7. When $r=0$, Eq. (18) is the Navier-Stokes on $(-4,0) \times \mathbb{R}^{3}$ once we assume the mean zero property for $u$.

Now we present two main local-study propositions which require the notion of (Problem II- $r$ ). These are kinds of partial regularity theorems for solutions of (Problem II- $r$ ). The main difficulty to prove them is that both $\bar{\eta}>0$ and $\bar{\delta}>0$ should be independent of any $r$ in $[0, \infty)$. We will prove this independence very carefully, which is the heart of Sections 3 and 4 .

\subsection{The first local study proposition for (Problem II-r)}

The following result is a quantitative version of partial regularity theorems which extends that of [42] up to $p=1$. The proof will be based on the De Giorgi iteration with a new pressure decomposition (see Lemma 3.3).

Proposition 2.1. There exists $\bar{\delta}>0$ with the following property:

If $u$ is a solution of (Problem II-r) for some $0 \leqslant r<\infty$ verifying both

$$
\begin{aligned}
& \|u\|_{L^{\infty}\left(-2,0 ; L^{2}\left(B\left(\frac{5}{4}\right)\right)\right)}+\|P\|_{L^{1}\left(-2,0 ; L^{1}(B(1))\right)}+\|\nabla u\|_{L^{2}\left(-2,0 ; L^{2}\left(B\left(\frac{5}{4}\right)\right)\right)} \leqslant \bar{\delta} \text { and } \\
& \|\mathcal{M}(|\nabla u|)\|_{L^{2}\left(-2,0 ; L^{2}(B(2))\right)} \leqslant \bar{\delta},
\end{aligned}
$$

then we have

$$
|u(t, x)| \leqslant 1 \quad \text { on }\left[-\frac{3}{2}, 0\right] \times B\left(\frac{1}{2}\right)
$$

Remark 2.8. For the case $r=0$, we do not need the smallness condition on $\|\mathcal{M}(|\nabla u|)\|_{L^{2}}$. Indeed, if $r=0$, then, in Lemma 3.5, we have $k_{r}=k_{0}=\infty$ without using the smallness of $\|\mathcal{M}(|\nabla u|)\|_{L^{2}}$. Then we can use Lemma 3.6 directly in order to get the above conclusion.

Remark 2.9. For the case $r>0$, the smallness condition on $\|\nabla u\|_{L^{2}}$ is not necessary because we have $|\nabla u(x)| \leqslant$ $M(|\nabla u|)(x)$ for a.e. $x$. 
The above proposition will be proved in Section 3. The two terms $\|u\|_{L_{t}^{\infty} L_{x}^{2}}$ and $\|P\|_{L_{t}^{1} L_{x}^{1}}$ do not have the correct scaling through the $\varepsilon$-zoom. The next Proposition 2.2 deals only quantities which have the correct scaling. However, since we cannot control the mean value of $u$ with such quantities, it will assume the mean zero property on $u$. As mentioned in the introduction, we cannot expect a local parabolic regularization effect if the drift is too big.

\subsection{The second local study proposition for (Problem II-r)}

Proposition 2.2. There exists $\bar{\eta}>0$, and there exists a family of constants $C_{d, \alpha}$ with the following property: If $u$ is a solution of (Problem II-r) for some $0 \leqslant r<\infty$ verifying both

$$
\begin{aligned}
& \int_{\mathbb{R}^{3}} \phi(x) u(t, x) d x=0 \quad \text { for } t \in(-4,0) \quad \text { and } \\
& \int_{-4}^{0} \int_{B(2)}\left(|\nabla u|^{2}(t, x)+\left|\nabla^{2} P\right|(t, x)+|\mathcal{M}(|\nabla u|)|^{2}(t, x)\right) d x d t \leqslant \bar{\eta},
\end{aligned}
$$

then $\left|\nabla^{d} u\right| \leqslant C_{d, 0}$ on $Q\left(\frac{1}{3}\right)=\left(-\left(\frac{1}{3}\right)^{2}, 0\right) \times B\left(\frac{1}{3}\right)$ for every integer $d \geqslant 0$.

Moreover if we assume further

$$
\begin{aligned}
& \int_{-4}^{0} \int_{B(2)}\left(|\mathcal{M}(\mathcal{M}(|\nabla u|))|^{2}+\left|\mathcal{M}\left(|\mathcal{M}(|\nabla u|)|^{q}\right)\right|^{2 / q}\right. \\
& \left.\quad+\left|\mathcal{M}\left(|\nabla u|^{q}\right)\right|^{2 / q}+\sum_{m=d}^{d+4} \sup _{\delta>0}\left(\left|\left(\nabla^{m-1} h^{\alpha}\right)_{\delta} * \nabla^{2} P\right|\right)\right) d x d t \leqslant \bar{\eta}
\end{aligned}
$$

for some integer $d \geqslant 1$ and for some real $\alpha \in(0,2)$ where $q=12 /(\alpha+6)$, then $\left|(-\Delta)^{\frac{\alpha}{2}} \nabla^{d} u\right| \leqslant C_{d, \alpha}$ on $Q\left(\frac{1}{6}\right)$ for $\operatorname{such}(d, \alpha)$.

Remark 2.10. The functions $h^{\alpha}$ and $\left(\nabla^{m-1} h^{\alpha}\right)_{\delta}$ are defined in (12).

Remark 2.11. For the case $r=0$, the smallness condition on $\|\mathcal{M}(|\nabla u|)\|_{L^{2}}$ in (22) is not necessary, while, for the case $r>0$, we do not need the smallness condition on $\|\nabla u\|_{L^{2}}$ (refer to Remarks 2.8, 2.9).

The proof will be given in Section 4 which will use the conclusion of the previous Proposition 2.1. Moreover we will use an induction argument together with the integral representation of the fractional Laplacian in order to get estimates for the fractional case. The Maximal function term of (22) is introduced to estimate non-local part of the velocity $u$ while the Maximal of Maximal function terms of (23) are to estimate non-local part of the drift velocity $w$, which depends on $u$ non-locally. On the other hand, because $\nabla^{2} P$ has only $L^{1}$ integrability, we cannot have $L^{1}$ Maximal function of $\nabla^{2} P$. Instead, we use some integrable function, which is the last term of (23). This term plays the role which captures non-local information of pressure (see (10)). These will be stated clearly in Sections 4 and 5 .

\section{Proof of the first local study Proposition 2.1}

This section is devoted to the proof of Proposition 2.1 which is a partial regularity theorem for (Problem II- $r$ ). Remember that we are looking for $\bar{\delta}$ which should be independent of $r \in[0, \infty)$.

In the first Section 3.1, we present some lemmas related to the convective velocity $w$ and a new pressure decomposition. Then, we prove two Lemmas 3.4 and 3.5 in Sections 3.2 and 3.3, which give us controls for large $r$ and small $r$, respectively. Finally, the proof of Proposition 2.1 is given in the last Section 3.4 where we combine those two lemmas. 


\subsection{A control on the convective velocity $w$ and a new pressure decomposition}

The following lemma says that the maximum of a convolution of any functions with $\phi_{r}$ can be controlled by just one point value of the Maximal function with some factor of $\frac{1}{r}$.

Lemma 3.1. Let $f$ be an integrable function in $\mathbb{R}^{3}$. Then for any integer $d \geqslant 0$, there exists $C=C(d)$ such that

$$
\left\|\nabla^{d}\left(f * \phi_{r}\right)\right\|_{L^{\infty}(B(2))} \leqslant \frac{C}{r^{d}} \cdot\left(1+\frac{4}{r}\right)^{3} \cdot \inf _{x \in B(2)} \mathcal{M} f(x)
$$

for any $0<r<\infty$.

Proof. Let $z, x \in B(2)$. Then, we compute

$$
\begin{aligned}
\left|\nabla^{d}\left(f * \phi_{r}\right)(z)\right| & =\left|\left(f * \nabla^{d} \phi_{r}\right)(z)\right|=\left|\int_{B(z, r)} f(y) \nabla^{d} \phi_{r}(z-y) d y\right| \\
& \leqslant\left\|\nabla^{d} \phi_{r}\right\|_{L^{\infty}} \int_{B(z, r)}|f(y)| d y=\frac{\left\|\nabla^{d} \phi\right\|_{L^{\infty}}}{r^{d+3}} \int_{B(z, r)}|f(y)| d y \\
& \leqslant \frac{\left\|\nabla^{d} \phi\right\|_{L^{\infty}}}{r^{d+3}} \frac{(r+4)^{3}}{(r+4)^{3}} \int_{B(x, r+4)}|f(y)| d y \leqslant \frac{C}{r^{d}} \cdot\left(1+\frac{4}{r}\right)^{3} \cdot \mathcal{M} f(x) .
\end{aligned}
$$

We used $B(z, r) \subset B(x, r+4)$. Then we take sup in $z$ and inf in $x$. Recall that $\phi(\cdot)$ is the fixed function in this paper.

The following corollary is just an application of the previous lemma to solutions of (Problem II- $r$ ).

Corollary 3.2. Let $u$ be a solution of (Problem II-r) for $0<r<\infty$. Then for any integer $d \geqslant 0$, there exists $C=C(d)$ such that

$$
\|w\|_{L^{2}\left(-4,0 ; L^{\infty}(B(2))\right)} \leqslant C \cdot\left(1+\frac{4}{r}\right)^{3} \cdot\|\mathcal{M}(|\nabla u|)\|_{L^{2}(Q(2))}
$$

and, for $d \geqslant 1$,

$$
\left\|\nabla^{d} w\right\|_{L^{2}\left(-4,0 ; L^{\infty}(B(2))\right)} \leqslant \frac{C}{r^{d-1}} \cdot\left(1+\frac{4}{r}\right)^{3} \cdot\|\mathcal{M}(|\nabla u|)\|_{L^{2}(Q(2))} .
$$

Proof. Recall $\int_{\mathbb{R}^{3}} w(t, y) \phi(y) d y=0$ and $\operatorname{supp}(\phi) \subset B(1)$. Thus, for $z \in B(2)$, we compute

$$
\begin{aligned}
|w(t, z)| & =\left|\int_{\mathbb{R}^{3}} w(t, z) \phi(y) d y-\int_{\mathbb{R}^{3}} w(t, y) \phi(y) d y\right| \\
& \leqslant\|\nabla w(t, \cdot)\|_{L^{\infty}(B(2))} \int_{\mathbb{R}^{3}}|z-y| \phi(y) d y \\
& \leqslant C\left\|(\nabla u) * \phi_{r}(t, \cdot)\right\|_{L^{\infty}(B(2))} \cdot \int_{\mathbb{R}^{3}} \phi(y) d y \\
& \leqslant C \cdot\left(1+\frac{4}{r}\right)^{3} \cdot \inf _{x \in B(2)} \mathcal{M}(|\nabla u|)(t, x) .
\end{aligned}
$$

For the last inequality, we used Lemma 3.1 to $\nabla u$. For $d \geqslant 1$, use $\nabla^{d} w=\nabla^{d-1}\left[(\nabla u) * \phi_{r}\right]$. 
To use a De Giorgi-type argument, we introduce the following notations, which will be used only in this section.

For real $k \geqslant 0$, we define

$$
\begin{aligned}
& B_{k}=\text { the ball in } \mathbb{R}^{3} \text { centered at the origin with radius } \frac{1}{2}\left(1+\frac{1}{2^{3 k}}\right), \\
& T_{k}=-\frac{1}{2}\left(3+\frac{1}{2^{k}}\right) \text {, } \\
& Q_{k}=\left[T_{k}, 0\right] \times B_{k} \text { and } \\
& s_{k}=\text { the distance between } B_{k-1}^{C} \text { and } B_{k-\frac{5}{6}} \\
& =2^{-3 k}((\sqrt{2}-1) 2 \sqrt{2}) \text {. }
\end{aligned}
$$

Also we define $s_{\infty}=0$. Note that $0<s_{1}<\frac{1}{4}$, and the sequence $\left\{s_{k}\right\}_{k=1}^{\infty}$ is strictly decreasing to zero as $k$ goes to $\infty$.

For each integer $k \geqslant 0$, we define $\psi_{k} \in C^{\infty}\left(\mathbb{R}^{3}\right)$ satisfying:

$$
\begin{aligned}
& \psi_{k}=1 \quad \text { in } B_{k-\frac{2}{3}}, \quad \psi_{k}=0 \quad \text { in } B_{k-\frac{5}{6}}^{C}, \\
& 0 \leqslant \psi_{k}(x) \leqslant 1, \quad\left|\nabla \psi_{k}(x)\right| \leqslant C 2^{3 k} \text { and }\left|\nabla^{2} \psi_{k}(x)\right| \leqslant C 2^{6 k} \text { for } x \in \mathbb{R}^{3} .
\end{aligned}
$$

This $\psi_{k}$ plays a role of a cut-off function for $B_{k}$.

To prove Proposition 2.1, we need the following important lemma related to a new pressure decomposition. Here we decompose our pressure term into three parts: a non-local part depending on $k$, a local part depending on $k$, and a non-local part, which does not depend on $k$. The last part will be absorbed into the velocity component later.

Lemma 3.3. There exists a constant $\Lambda_{1}>0$ with the following property:

Suppose $A_{i j} \in L^{1}\left(B_{0}\right), 1 \leqslant i, j \leqslant 3$ and $P \in L^{1}\left(B_{0}\right)$ with $-\Delta P=\sum_{i j} \partial_{i} \partial_{j} A_{i j}$ in $B_{0}$. Then, there exist a function $P_{3}$ with $\left.P_{3}\right|_{B_{\frac{2}{3}}} \in L^{\infty}$ such that, for any $k \geqslant 1$, we can decompose $P$ by

$$
P=P_{1, k}+P_{2, k}+P_{3} \quad \text { in } B_{\frac{1}{3}},
$$

and they satisfy

$$
\begin{aligned}
& \left\|\nabla P_{1, k}\right\|_{L^{\infty}\left(B_{k-\frac{1}{3}}\right)}+\left\|P_{1, k}\right\|_{L^{\infty}\left(B_{k-\frac{1}{3}}\right)} \leqslant \Lambda_{1} 2^{12 k} \sum_{i j}\left\|A_{i j}\right\|_{L^{1}\left(B_{\frac{1}{6}}\right)}, \\
& -\Delta P_{2, k}=\sum_{i j} \partial_{i} \partial_{j}\left(\psi_{k} A_{i j}\right) \quad \text { in } \mathbb{R}^{3} \text { and } \\
& \left\|\nabla P_{3}\right\|_{L^{\infty}\left(B_{\frac{2}{3}}\right)} \leqslant \Lambda_{1}\left(\|P\|_{L^{1}\left(B_{\frac{1}{6}}\right)}+\sum_{i j}\left\|A_{i j}\right\|_{L^{1}\left(B_{\frac{1}{6}}\right)}\right) .
\end{aligned}
$$

Note that $\Lambda_{1}$ is an independent constant.

Proof. The product rule and the hypothesis imply

$$
\begin{aligned}
-\Delta\left(\psi_{1} P\right) & =-\psi_{1} \Delta P-2 \operatorname{div}\left(\left(\nabla \psi_{1}\right) P\right)+P \Delta \psi_{1} \\
& =\psi_{1} \sum_{i j} \partial_{i} \partial_{j} A_{i j}-2 \operatorname{div}\left(\left(\nabla \psi_{1}\right) P\right)+P \Delta \psi_{1} \\
& =-\Delta P_{1, k}-\Delta P_{2, k}-\Delta P_{3}
\end{aligned}
$$

where $P_{1, k}, P_{2, k}$ and $P_{3}$ are defined by

$$
\begin{aligned}
& -\Delta P_{1, k}=\sum_{i j} \partial_{i} \partial_{j}\left(\left(\psi_{1}-\psi_{k}\right) A_{i j}\right), \\
& -\Delta P_{2, k}=\sum_{i j} \partial_{i} \partial_{j}\left(\psi_{k} A_{i j}\right),
\end{aligned}
$$




$$
-\Delta P_{3}=-\sum_{i j} \partial_{j}\left[\left(\partial_{i} \psi_{1}\right)\left(A_{i j}\right)\right]-\sum_{i j} \partial_{i}\left[\left(\partial_{j} \psi_{1}\right)\left(A_{i j}\right)\right]+\sum_{i j}\left(\partial_{i} \partial_{j} \psi_{1}\right)\left(A_{i j}\right)-2 \operatorname{div}\left(\left(\nabla \psi_{1}\right) P\right)+P \Delta \psi_{1}
$$

Here, $P_{1, k}$ and $P_{3}$ are defined by the representation formula $(-\Delta)^{-1}(f)=\frac{1}{4 \pi}\left(\frac{1}{|x|} * f\right)$ while $P_{2, k}$ by the Riesz transforms.

Since $\psi_{1}=1$ on $B_{\frac{1}{3}}$, we have $\Delta P=\Delta\left(\psi_{1} P\right)$ on $B_{\frac{1}{3}}$. Thus (26) holds.

By definition of $P_{2, k}$, (28) holds.

For (27) and (29), it follows from the proof of Lemma 3 of [42] directly. For completeness, we present the proof. Note that $\left(\psi_{1}-\psi_{k}\right)$ is supported in $\left(B_{\frac{1}{6}}-B_{k-\frac{2}{3}}\right)$ and $\nabla \psi_{1}$ is supported in $\left(B_{\frac{1}{6}}-B_{\frac{1}{3}}\right)$. Thus for $x \in B_{k-\frac{1}{3}}$, we get

$$
\begin{aligned}
\left|P_{1, k}(x)\right| & =\left|\frac{1}{4 \pi} \int_{\left(B_{\frac{1}{6}}-B_{\left.k-\frac{2}{3}\right)}\right.} \frac{1}{|x-y|} \sum_{i j}\left(\partial_{i} \partial_{j}\left(\left(\psi_{1}-\psi_{k}\right) A_{i j}\right)\right)(y) d y\right| \\
& \leqslant \sup _{y \in B_{k-\frac{2}{3}}^{C}}\left(\left|\nabla_{y}^{2} \frac{1}{|x-y|}\right|\right) \cdot \sum_{i j} \int_{B_{\frac{1}{6}}}\left|A_{i j}(x)\right| d y \\
& \leqslant C \cdot \sup _{y \in B_{k-\frac{2}{3}}^{C}}\left(\frac{1}{|x-y|^{3}}\right) \cdot \sum_{i j}\left\|A_{i j}\right\|_{L^{1}\left(B_{\frac{1}{6}}\right)} \leqslant C_{1} \cdot 2^{9 k} \cdot \sum_{i j}\left\|A_{i j}\right\|_{L^{1}\left(B_{\frac{1}{6}}\right)} .
\end{aligned}
$$

We used integration by parts with the facts $|x-y| \geqslant 2^{-3 k}$ and $\left|\left(\psi_{1}-\psi_{k}\right)\right| \leqslant 1$.

In the same way, for $x \in B_{k-\frac{1}{3}}$, we compute

$$
\left|\nabla P_{1, k}(x)\right| \leqslant C_{2} \cdot 2^{12 k} \cdot \sum_{i j}\left\|A_{i j}\right\|_{L^{1}\left(B_{\frac{1}{6}}\right)} .
$$

For $x \in B_{\frac{2}{3}}$, we get

$$
\begin{aligned}
\left|\nabla P_{3}(x)\right|= & \mid \frac{1}{4 \pi} \int_{\left(B_{\frac{1}{6}}-B_{\frac{1}{3}}\right)}\left(\nabla_{y} \frac{1}{|x-y|}\right)\left[-\sum_{i j} \partial_{j}\left[\left(\partial_{i} \psi_{1}\right)\left(A_{i j}\right)\right]-\sum_{i j} \partial_{i}\left[\left(\partial_{j} \psi_{1}\right)\left(A_{i j}\right)\right]\right. \\
& \left.+\sum_{i j}\left(\partial_{i} \partial_{j} \psi_{1}\right)\left(A_{i j}\right)-2 \operatorname{div}\left(\left(\nabla \psi_{1}\right) P\right)+P \Delta \psi_{1}\right] d y \mid \\
\leqslant & C_{3}\left(\sum_{i j}\left\|A_{i j}\right\|_{L^{1}\left(B_{\frac{1}{6}}\right)}+\|P\|_{L^{1}\left(B_{\frac{1}{6}}\right)}\right) .
\end{aligned}
$$

These prove (27) and (29), and we keep the constant $\Lambda_{1}=\max \left(C_{1}, C_{2}, C_{3}\right)$ for the future use.

Before presenting the De Giorgi arguments for large $r$ and small $r$, we need more notations. In the following two main Lemmas 3.4 and 3.5, $P_{3}$ will be constructed from solutions $(u, P$ ) for (Problem II- $r$ ) by using the previous Lemma 3.3 and it will be clearly shown that $\nabla P_{3}$ has the $L_{t}^{1} L_{x}^{\infty}$ bound. Thus we can define, for $t \in[-2,0]$ and for $k \geqslant 0$,

$$
E_{k}(t)=\frac{1}{2}\left(1-2^{-k}\right)+\int_{-1}^{t}\left\|\nabla P_{3}(s, \cdot)\right\|_{L^{\infty}\left(B_{\frac{2}{3}}\right)} d s .
$$

Note that $E_{k}$ depends on $t$. We also define

$$
\begin{aligned}
v_{k} & =\left(|u|-E_{k}\right)_{+}, \\
d_{k} & =\sqrt{\frac{E_{k} \mathbf{1}_{\left\{|u| \geqslant E_{k}\right\}}|\nabla| u||^{2}+\frac{v_{k}}{|u|}|\nabla u|^{2}}{|u|}} \text { and }
\end{aligned}
$$




$$
\begin{aligned}
U_{k} & =\sup _{t \in\left[T_{k}, 0\right]}\left(\int_{B_{k}}\left|v_{k}\right|^{2} d x\right)+\iint_{Q_{k}}\left|d_{k}\right|^{2} d x d t \\
& =\left\|v_{k}\right\|_{L^{\infty}\left(T_{k}, 0 ; L^{2}\left(B_{k}\right)\right)}^{2}+\left\|d_{k}\right\|_{L^{2}\left(Q_{k}\right)}^{2} .
\end{aligned}
$$

It will be shown that $P_{3}$ can be absorbed into $v_{k}$, which is the key idea of the proof of Proposition 2.1.

\subsection{De Giorgi argument to get a control for large $r$}

The following lemma says that we can obtain a certain uniform non-linear estimate in the form of $W_{k} \leqslant C^{k} \cdot W_{k-1}^{\beta}$ when $r$ is large. Then an elementary lemma can give us the conclusion (we will see Lemma 3.6 later). However, for small $r$, the factor $\left(1 / r^{3}\right)$ blows up as $r$ goes to zero. The case of small $r$ will be treated in Lemma 3.5.

Lemma 3.4. There exist constants $\delta_{1}>0$ and $\bar{C}_{1}>1$ such that if $u$ is a solution of (Problem II-r) for some $0<r<\infty$ verifying

$$
\|u\|_{L^{\infty}\left(-2,0 ; L^{2}\left(B\left(\frac{5}{4}\right)\right)\right)}+\|P\|_{L^{1}\left(-2,0 ; L^{1}(B(1))\right)}+\|\mathcal{M}(|\nabla u|)\|_{L^{2}\left(-2,0 ; L^{2}(B(2))\right)} \leqslant \delta_{1},
$$

then we have

$$
U_{k} \leqslant \begin{cases}\left(\bar{C}_{1}\right)^{k} U_{k-1}^{\frac{7}{6}}, & \text { for any } k \geqslant 1 \text { if } r \geqslant s_{1}, \\ \frac{1}{r^{3}} \cdot\left(\bar{C}_{1}\right)^{k} U_{k-1}^{\frac{7}{6}}, & \text { for any } k \geqslant 1 \text { if } r<s_{1} .\end{cases}
$$

Remark 3.1. Since $|f(x)| \leqslant M(f)(x)$ almost everywhere, the above assumption implies $\|\nabla u\|_{L^{2}\left(-2,0 ; L^{2}(B(2))\right)} \leqslant \delta_{1}$.

Remark 3.2. The parameter $s_{1}$ is the fixed constant defined in (24) such that $0<s_{1}<1 / 4$, and $\left(\delta_{1}, \bar{C}_{1}\right)$ is independent of any $0<r<\infty$. It will be clear that the exponent $7 / 6$ is not optimal and we can make it close to (4/3) arbitrarily. However, any exponent bigger than 1 is enough for our study.

Proof of Lemma 3.4. We assume $\delta_{1}<1$. First we claim that there exists a constant $\Lambda_{2} \geqslant 1$ such that

$$
\||w| \cdot|u|\|_{L^{2}\left(-2,0 ; L^{3 / 2}\left(B_{\left.\frac{1}{6}\right)}\right)\right.} \leqslant \Lambda_{2} \cdot \delta_{1} \quad \text { for any } 0<r<\infty .
$$

In order to prove the above claim (31), we separate it into (I)-large $r$ case $\left(r \geqslant s_{1}\right)$ and (II)-small $r$ case $\left(r<s_{1}\right)$ :

(I)-large $r$ case. From Corollary 3.2 if $r \geqslant s_{1}$, then we get

$$
\begin{aligned}
\|w\|_{L^{2}\left(-4,0 ; L^{\infty}(B(2))\right)} & \leqslant C \cdot\left(1+\frac{4}{s_{1}}\right)^{3} \cdot\|\mathcal{M}(|\nabla u|)\|_{L^{2}(Q(2))} \\
& \leqslant C\|\mathcal{M}(|\nabla u|)\|_{L^{2}(Q(2))} \leqslant C \delta_{1} .
\end{aligned}
$$

Likewise, we obtain

$$
\|\nabla w\|_{L^{2}\left(-4,0 ; L^{\infty}(B(2))\right)} \leqslant C \delta_{1} .
$$

With Hölder's inequality and $B_{\frac{1}{6}} \subset B_{0}=B(1) \subset B\left(\frac{5}{4}\right) \subset B(2)$, we get

$$
\begin{aligned}
\||w| \cdot|u|\|_{L^{2}\left(-2,0 ; L^{3 / 2}\left(B_{\frac{1}{6}}\right)\right)} & \leqslant C\|u\|_{L^{\infty}\left(-2,0 ; L^{2}\left(B\left(\frac{5}{4}\right)\right)\right)} \cdot\|w\|_{L^{2}\left(-4,0 ; L^{\infty}(B(2))\right)} \\
& \leqslant C \cdot \delta_{1}{ }^{2} \leqslant C_{1} \cdot \delta_{1},
\end{aligned}
$$

so we obtained (31) for $r \geqslant s_{1}$.

(II)-small $r$ case. On the other hand, if $r<s_{1}$, then we get

$$
\begin{aligned}
\|w\|_{L^{2}\left(-4,0 ; L^{\infty}(B(2))\right)} & \leqslant C \cdot\left(1+\frac{4}{r}\right)^{3} \cdot\|\mathcal{M}(|\nabla u|)\|_{L^{2}(Q(2))} \\
& \leqslant C \frac{1}{r^{3}}\|\mathcal{M}(|\nabla u|)\|_{L^{2}(Q(2))} \leqslant C \frac{1}{r^{3}} \delta_{1}
\end{aligned}
$$


and

$$
\|\nabla w\|_{L^{2}\left(-4,0 ; L^{\infty}(B(2))\right)} \leqslant C \frac{1}{r^{3}} \delta_{1} .
$$

However, it is not enough to prove (31) because the factor $\frac{1}{r^{3}}$ blows up as $r$ goes to zero. Instead, we use the fact that $w$ and $u$ are similar for small $r$ in the following sense:

$$
\|u\|_{L^{4}\left(-2,0 ; L^{3}\left(B_{0}\right)\right)} \leqslant C\left(\|u\|_{L^{\infty}\left(-2,0 ; L^{2}\left(B_{0}\right)\right)}+\|\nabla u\|_{L^{2}\left(-2,0 ; L^{2}\left(B_{0}\right)\right)}\right) \leqslant C \delta_{1}
$$

and

$$
\left\|w^{\prime}\right\|_{L^{4}\left(-2,0 ; L^{3}\left(B_{\frac{1}{6}}\right)\right)}=\left\|u * \phi_{r}\right\|_{L^{4}\left(-2,0 ; L^{3}\left(B_{\frac{1}{6}}\right)\right)} \leqslant\|u\|_{L^{4}\left(-2,0 ; L^{3}\left(B_{0}\right)\right)} \leqslant C \delta_{1}
$$

because $u * \phi_{r}$ in $B_{\frac{1}{6}}$ depends only on $u$ in $B_{0}$ (recall that $r \leqslant s_{1}$ and $s_{1}$ is the distance between $B_{0}^{C}$ and $B_{\frac{1}{6}}$ and refer to (8)). For $w^{\prime \prime}$, we compute

$$
\begin{aligned}
\left\|w^{\prime \prime}\right\|_{L^{\infty}\left(-2,0 ; L^{\infty}(B(2))\right)} & =\left\|w^{\prime \prime}\right\|_{L_{t}^{\infty}((-2,0))} \\
& =\left\|\int_{\mathbb{R}^{3}} \phi(y)\left(u * \phi_{r}\right)(y) d y\right\|_{L_{t}^{\infty}((-2,0))} \\
& \leqslant C\|\| u * \phi_{r}\left\|_{L_{x}^{1}(B(1))}\right\|_{L_{t}^{\infty}((-2,0))} \\
& \leqslant C\|\| u\left\|_{L_{x}^{1}\left(B\left(\frac{5}{4}\right)\right)}\right\|_{L_{t}^{\infty}((-2,0))} \\
& \leqslant C\|u\|_{L^{\infty}\left(-2,0 ; L^{2}\left(B\left(\frac{5}{4}\right)\right)\right)} \\
& \leqslant C \delta_{1}
\end{aligned}
$$

because $w^{\prime \prime}$ is a constant in $x$, and $u * \phi_{r}$ in $B(1)$ depends only on $u$ in $B\left(1+s_{1}\right)$ which is a subset of $B\left(\frac{5}{4}\right)$. As a result, we have

$$
\begin{aligned}
\||w| \cdot|u|\|_{L^{2}\left(-2,0 ; L^{3 / 2}\left(B_{\left.\left.\frac{1}{6}\right)\right)}\right.\right.} & \leqslant C\|u\|_{L^{4}\left(-2,0 ; L^{3}(B(1))\right)} \cdot\|w\|_{L^{4}\left(-2,0 ; L^{3}\left(B\left(\frac{1}{6}\right)\right)\right)} \\
& \leqslant C \delta_{1} \cdot\left\|\left|w^{\prime}\right|+\left|w^{\prime \prime}\right|\right\|_{L^{4}\left(-2,0 ; L^{3}\left(B\left(\frac{1}{6}\right)\right)\right)} \\
& \leqslant C \cdot \delta_{1}{ }^{2} \leqslant C_{2} \cdot \delta_{1}
\end{aligned}
$$

so that we obtained (31) for $r \leqslant s_{1}$.

Hence, by taking

$$
\Lambda_{2}=\max \left(C_{1}, C_{2}, 1\right)
$$

we have (31), and $\Lambda_{2}$ is independent of $0<r<\infty$ as long as $\delta_{1}<1$. From now on, we assume $\delta_{1}<1$ sufficiently small to satisfy $10 \cdot \Lambda_{1} \cdot \Lambda_{2} \cdot \delta_{1} \leqslant 1 / 2$ (recall that $\Lambda_{1}$ comes from Lemma 3.3).

Thanks to Lemma 3.3 and (31), by putting $A_{i j}=w_{i} u_{j}$, we can decompose $P$ by

$$
P=P_{1, k}+P_{2, k}+P_{3} \quad \text { in }[-2,0] \times B_{\frac{1}{3}}
$$

for each $k \geqslant 1$ with the following properties: for any $k \geqslant 1$,

$$
\begin{gathered}
\left\|\left|\nabla P_{1, k}\right|+\left|P_{1, k}\right|\right\|_{L^{2}\left(-2,0 ; L^{\infty}\left(B_{k-\frac{1}{3}}\right)\right)} \leqslant \Lambda_{1} 2^{12 k} \sum_{i j}\left\|w_{i} u_{j}\right\|_{L^{2}\left(-2,0 ; L^{1}\left(B_{\frac{1}{6}}\right)\right)} \\
\leqslant 9 \cdot \Lambda_{1} \cdot \Lambda_{2} \cdot \delta_{1} \cdot 2^{12 k} \leqslant 2^{12 k}, \\
-\Delta P_{2, k}=\sum_{i j} \partial_{i} \partial_{j}\left(\psi_{k} w_{i} u_{j}\right) \quad \text { in }[-2,0] \times \mathbb{R}^{3} \text { and } \\
\left\|\nabla P_{3}\right\|_{L^{1}\left(-2,0 ; L^{\infty}\left(B_{\left.\frac{2}{3}\right)}\right.\right.} \leqslant \Lambda_{1}\left(\|P\|_{L^{1}\left(-2,0 ; L^{1}(B(1))\right)}+\sum_{i j}\left\|w_{i} u_{j}\right\|_{\left.L^{2}\left(-2,0 ; L^{1}(B(1))\right)\right)}\right) \\
\leqslant \Lambda_{1}\left(\delta_{1}+9 \cdot \Lambda_{2} \cdot \delta_{1}\right) \leqslant 10 \cdot \Lambda_{1} \cdot \Lambda_{2} \cdot \delta_{1} \leqslant \frac{1}{2} .
\end{gathered}
$$

Note that (41) enables $E_{k}$ to be well-defined and it satisfies $0 \leqslant E_{k} \leqslant 1$ (see the definition of $E_{k}$ in (30)). 
In the following Remarks 3.3-3.5, we gather some easy results, which were obtained in [42], without a proof. They can be found in Lemmas 4, 6 and the remark of Lemma 4 of [42]. Note that any constants $C$ in the following remarks do not depend on $k$.

Remark 3.3. For any $k \geqslant 0$, the function $u$ can be decomposed by $u=u \frac{v_{k}}{|u|}+u\left(1-\frac{v_{k}}{|u|}\right)$. Also we have

$$
\begin{aligned}
& \left|u\left(1-\frac{v_{k}}{|u|}\right)\right| \leqslant 1, \quad \frac{v_{k}}{|u|}|\nabla u| \leqslant d_{k}, \quad \mathbf{1}_{|u| \geqslant E_{k}}|\nabla| u|| \leqslant d_{k}, \\
& \left|\nabla v_{k}\right| \leqslant d_{k} \quad \text { and } \quad\left|\nabla \frac{u v_{k}}{|u|}\right| \leqslant 3 d_{k} .
\end{aligned}
$$

Remark 3.4. For any $k \geqslant 1$ and for any $q \geqslant 1$, we have

$$
\left\|\mathbf{1}_{v_{k}>0}\right\|_{L^{q}\left(Q_{k-1}\right)} \leqslant C 2^{\frac{10 k}{3 q}} U_{k-1}^{\frac{5}{3 q}} \quad \text { and } \quad\left\|\mathbf{1}_{v_{k}>0}\right\|_{L^{\infty}\left(T_{k-1}, 0 ; L^{q}\left(Q_{k-1}\right)\right)} \leqslant C 2^{\frac{2 k}{q}} U_{k-1}^{\frac{1}{q}} .
$$

Remark 3.5. For any $k \geqslant 1$, we have $\left\|v_{k-1}\right\|_{L^{\frac{10}{3}}\left(Q_{k-1}\right)} \leqslant C U_{k-1}^{\frac{1}{2}}$.

By using the above Remarks 3.3-3.5, we have, for any $1 \leqslant p \leqslant \frac{10}{3}$,

$$
\begin{aligned}
\left\|v_{k}\right\|_{L^{p}\left(Q_{k-1}\right)} & =\left\|v_{k} \mathbf{1}_{v_{k}>0}\right\|_{L^{p}\left(Q_{k-1}\right)} \\
& \leqslant\left\|v_{k}\right\|_{L^{\frac{10}{3}}\left(Q_{k-1}\right)} \cdot\left\|\mathbf{1}_{v_{k}>0}\right\|_{L^{1 /\left(\frac{1}{p}-\frac{3}{10}\right)}\left(Q_{k-1}\right)} \\
& \leqslant\left\|v_{k-1}\right\|_{L^{\frac{10}{3}}\left(Q_{k-1}\right)} \cdot C 2^{\frac{10 k}{3} \cdot\left(\frac{1}{p}-\frac{3}{10}\right)} U_{k-1}^{\frac{5}{3} \cdot\left(\frac{1}{p}-\frac{3}{10}\right)} \\
& \leqslant C 2^{\frac{7 k}{3}} U_{k-1}^{\frac{5}{3 p}} .
\end{aligned}
$$

Likewise, we get, for any $1 \leqslant p \leqslant 2$,

$$
\left\|v_{k}\right\|_{L^{\infty}\left(T_{k-1}, 0 ; L^{p}\left(B_{k-1}\right)\right)} \leqslant C 2^{k} U_{k-1}^{\frac{1}{p}}
$$

and

$$
\left\|d_{k}\right\|_{L^{p}\left(Q_{k-1}\right)} \leqslant C 2^{\frac{5 k}{3}} U_{k-1}^{\frac{5}{3 p}-\frac{1}{3}}
$$

Second, we claim that for every $k \geqslant 1$, the function $v_{k}$ verifies

$$
\partial_{t} \frac{v_{k}^{2}}{2}+\operatorname{div}\left(w \frac{v_{k}^{2}}{2}\right)+d_{k}^{2}-\Delta \frac{v_{k}^{2}}{2}+\operatorname{div}\left(u\left(P_{1, k}+P_{2, k}\right)\right)+\left(\frac{v_{k}}{|u|}-1\right) u \cdot \nabla\left(P_{1, k}+P_{2, k}\right) \leqslant 0
$$

in $(-2,0) \times B_{\frac{2}{3}}$.

Remark 3.6. The above inequality (46) does not contain $P_{3}$. We will see that this fact comes from the definition of $E_{k}(t)$ in $(30)$.

Indeed, observe that $\frac{v_{k}^{2}}{2}=\frac{|u|^{2}}{2}+\frac{v_{k}^{2}-|u|^{2}}{2}$ and note that $E_{k}$ does not depend on the space variable but on the time variable. Thus we compute, for time derivatives,

$$
\begin{aligned}
\partial_{t}\left(\frac{v_{k}^{2}-|u|^{2}}{2}\right) & =v_{k} \partial_{t} v_{k}-u \partial_{t} u=v_{k} \partial_{t}|u|-v_{k} \partial_{t} E_{k}-u \partial_{t} u \\
& =u\left(\frac{v_{k}}{|u|}-1\right) \partial_{t} u-v_{k} \partial_{t} E_{k}=u\left(\frac{v_{k}}{|u|}-1\right) \partial_{t} u-v_{k}\left\|\nabla P_{3}(t, \cdot)\right\|_{L^{\infty}\left(B_{\frac{2}{3}}\right)}
\end{aligned}
$$

while, for any space derivatives $\partial_{\alpha}$, we get 


$$
\partial_{\alpha}\left(\frac{v_{k}^{2}-|u|^{2}}{2}\right)=u\left(\frac{v_{k}}{|u|}-1\right) \partial_{\alpha} u .
$$

Then we follow the same way as Lemma 5 of [42] did: First, we multiply (18) by $u\left(\frac{v_{k}}{|u|}-1\right)$, and then we sum the result and (20). We omit the details. As a result, we have

$$
\begin{aligned}
0 \geqslant & \partial_{t} \frac{v_{k}^{2}}{2}+\operatorname{div}\left(w \frac{v_{k}^{2}}{2}\right)+d_{k}^{2}-\Delta \frac{v_{k}^{2}}{2}+v_{k}\left\|\nabla P_{3}(t, \cdot)\right\|_{L^{\infty}\left(B_{\frac{2}{3}}\right)}+\operatorname{div}(u P)+\left(\frac{v_{k}}{|u|}-1\right) u \cdot \nabla P \\
= & \partial_{t} \frac{v_{k}^{2}}{2}+\operatorname{div}\left(w \frac{v_{k}^{2}}{2}\right)+d_{k}^{2}-\Delta \frac{v_{k}^{2}}{2}+\left(v_{k}\left\|\nabla P_{3}(t, \cdot)\right\|_{L^{\infty}\left(B_{\frac{2}{3}}\right)}+\frac{v_{k}}{|u|} u \cdot \nabla P_{3}\right) \\
& +\operatorname{div}\left(u\left(P_{1, k}+P_{2, k}\right)\right)+\left(\frac{v_{k}}{|u|}-1\right) u \cdot \nabla\left(P_{1, k}+P_{2, k}\right) .
\end{aligned}
$$

For the last equality, we used the fact $P=P_{1, k}+P_{2, k}+P_{3}$ in $B_{\frac{1}{3}}$ and

$$
\operatorname{div}\left(u P_{3}\right)+\left(\frac{v_{k}}{|u|}-1\right) u \cdot \nabla P_{3}=\frac{v_{k}}{|u|} u \cdot \nabla P_{3} .
$$

Thus we proved the claim (46) due to

$$
v_{k}\left\|\nabla P_{3}(t, \cdot)\right\|_{L^{\infty}\left(B_{\frac{2}{3}}\right)}+\frac{v_{k}}{|u|} u \cdot \nabla P_{3} \geqslant 0 \quad \text { on }(-2,0) \times B_{\frac{2}{3}} .
$$

For any integer $k$, we introduce a cut-off function $\eta_{k}(x) \in C^{\infty}\left(\mathbb{R}^{3}\right)$ satisfying

$$
\begin{aligned}
& \eta_{k}=1 \quad \text { in } B_{k}, \quad \eta_{k}=0 \quad \text { in } B_{k-\frac{1}{3}}^{C}, 0 \leqslant \eta_{k} \leqslant 1, \\
& \left|\nabla \eta_{k}\right| \leqslant C 2^{3 k} \text { and }\left|\nabla^{2} \eta_{k}\right| \leqslant C 2^{6 k}, \text { for any } x \in \mathbb{R}^{3} .
\end{aligned}
$$

We multiply (46) by $\eta_{k}$ and integrate on $[\sigma, t] \times \mathbb{R}^{3}$ for $T_{k-1} \leqslant \sigma \leqslant T_{k} \leqslant t \leqslant 0$ to get:

$$
\begin{aligned}
& \int_{\mathbb{R}^{3}} \eta_{k}(x) \frac{\left|v_{k}(t, x)\right|^{2}}{2} d x+\int_{\sigma} \int_{\mathbb{R}^{3}} \eta_{k}(x) d_{k}^{2}(s, x) d x d s \\
& \leqslant \int_{\mathbb{R}^{3}} \eta_{k}(x) \frac{\left|v_{k}(\sigma, x)\right|^{2}}{2} d x+\int_{\sigma}^{t} \int_{\mathbb{R}^{3}}\left(\nabla \eta_{k}\right)(x) w(s, x) \frac{\left|v_{k}(s, x)\right|^{2}}{2} d x d s+\int_{\sigma}^{t} \int_{\mathbb{R}^{3}}\left(\Delta \eta_{k}\right)(x) \frac{\left|v_{k}(s, x)\right|^{2}}{2} d x d s \\
& \quad-\int_{\sigma}^{t} \int_{\mathbb{R}^{3}} \eta_{k}(x)\left(\operatorname{div}\left(u\left(P_{1, k}+P_{2, k}\right)\right)+\left(\frac{v_{k}}{|u|}-1\right) u \cdot \nabla\left(P_{1, k}+P_{2, k}\right)\right)(s, x) d x d s .
\end{aligned}
$$

We integrate on $\sigma \in\left[T_{k-1}, T_{k}\right]$ and divide by $-\left(T_{k-1}-T_{k}\right)=2^{-(k+1)}$ to get:

$$
\begin{aligned}
& \sup _{t \in\left[T_{k}, 1\right]}\left(\int_{\mathbb{R}^{3}} \eta_{k}(x) \frac{\left|v_{k}(t, x)\right|^{2}}{2} d x+\int_{T_{k}}^{t} \int_{\mathbb{R}^{3}} \eta_{k}(x) d_{k}^{2}(s, x) d x d s\right) \\
& \leqslant 2^{k+1} \cdot \int_{T_{k-1}}^{T_{k}} \int_{\mathbb{R}^{3}} \eta_{k}(x) \frac{\left|v_{k}(\sigma, x)\right|^{2}}{2} d x \\
& \quad+\int_{T_{k-1}}^{0}\left|\int_{\mathbb{R}^{3}} \nabla \eta_{k}(x) w(s, x) \frac{\left|v_{k}(s, x)\right|^{2}}{2} d x\right| d s+\int_{T_{k-1}}^{0}\left|\int_{\mathbb{R}^{3}} \Delta \eta_{k}(x) \frac{\left|v_{k}(s, x)\right|^{2}}{2} d x\right| d s
\end{aligned}
$$




$$
+\int_{T_{k-1}}^{0}\left|\int_{\mathbb{R}^{3}} \eta_{k}(x)\left(\operatorname{div}\left(u\left(P_{1, k}+P_{2, k}\right)\right)+\left(\frac{v_{k}}{|u|}-1\right) u \cdot \nabla\left(P_{1, k}+P_{2, k}\right)\right)(s, x) d x\right| d s .
$$

From $\eta_{k}=1$ on $B_{k}$, we obtain

$$
\begin{aligned}
U_{k} & \leqslant \sup _{t \in\left[T_{k}, 1\right]}\left(\int_{\mathbb{R}^{3}} \eta_{k}(x) \frac{\left|v_{k}(t, x)\right|^{2}}{2} d x\right)+\int_{T_{k}}^{0} \int_{\mathbb{R}^{3}} \eta_{k}(x) d_{k}^{2}(s, x) d x d s \\
& \leqslant 2 \cdot \sup _{t \in\left[T_{k}, 1\right]}\left(\int_{\mathbb{R}^{3}} \eta_{k}(x) \frac{\left|v_{k}(t, x)\right|^{2}}{2} d x+\int_{T_{k}} \int_{\mathbb{R}^{3}} \eta_{k}(x) d_{k}^{2}(s, x) d x d s\right) .
\end{aligned}
$$

Thus we have

$$
U_{k} \leqslant(I)+(I I)+(I I I)+(I V)
$$

where

$$
\begin{aligned}
& (I)=C 2^{6 k} \int_{Q_{k-1}}\left|v_{k}(s, x)\right|^{2} d x d s, \\
& (I I)=\int_{Q_{k-1}}\left|\nabla \eta_{k}(x)\right| \cdot|w(s, x)| \cdot\left|v_{k}(s, x)\right|^{2} d x d s, \\
& (I I I)=2 \int_{T_{k-1}}^{0}\left|\int_{\mathbb{R}^{3}} \eta_{k}(x)\left(\operatorname{div}\left(u P_{1, k}\right)+\left(\frac{v_{k}}{|u|}-1\right) u \cdot \nabla P_{1, k}\right)(s, x) d x\right| d s \quad \text { and } \\
& (I V)=2 \int_{T_{k-1}}^{0}\left|\int_{\mathbb{R}^{3}} \eta_{k}(x)\left(\operatorname{div}\left(u P_{2, k}\right)+\left(\frac{v_{k}}{|u|}-1\right) u \cdot \nabla P_{2, k}\right)(s, x) d x\right| d s .
\end{aligned}
$$

For (I), by using (43), we get, for any $0<r<\infty$,

$$
(I)=C 2^{6 k}\left\|v_{k}\right\|_{L^{2}\left(Q_{k-1}\right)}^{2} \leqslant C 2^{10 k} U_{k-1}^{\frac{5}{3}} .
$$

For (II) with $r \geqslant s_{1}$, by using (32) and (44), we compute

$$
\begin{aligned}
(I I) & \leqslant C 2^{3 k}\|w\|_{L^{2}\left(-4,0 ; L^{\infty}(B(2))\right)} \cdot\left\|\left|v_{k}\right|^{2}\right\|_{L^{2}\left(T_{k-1}, 0 ; L^{1}\left(B_{k-1}\right)\right)} \\
& \leqslant C 2^{3 k} \delta_{1}\left\|v_{k}\right\|_{L^{\infty}\left(T_{k-1}, 0 ; L^{\frac{6}{5}}\left(B_{k-1}\right)\right)} \cdot\left\|v_{k}\right\|_{L^{2}\left(T_{k-1}, 0 ; L^{6}\left(B_{k-1}\right)\right)} \\
& \leqslant C 2^{4 k} \delta_{1} U_{k-1}^{\frac{5}{6}} \cdot\left(\left\|v_{k-1}\right\|_{L^{\infty}\left(T_{k-1}, 0 ; L^{2}\left(B_{k-1}\right)\right)}+\left\|\nabla v_{k-1}\right\|_{L^{2}\left(T_{k-1}, 0 ; L^{2}\left(B_{k-1}\right)\right)}\right) \\
& \leqslant C 2^{4 k} \cdot \delta_{1} \cdot U_{k-1}^{\frac{5}{6}} \cdot U_{k-1}^{\frac{1}{2}} \leqslant C 2^{4 k} \cdot \delta_{1} \cdot U_{k-1}^{\frac{4}{3}} \leqslant C 2^{4 k} \cdot U_{k-1}^{\frac{4}{3}} \cdot
\end{aligned}
$$

For $r<s_{1}$, we follow the above steps using (34) instead of (32), then we get

$$
(I I) \leqslant C \frac{1}{r^{3}} 2^{4 k} \cdot U_{k-1}^{\frac{4}{3}} .
$$

For (III) (non-local pressure term), thanks to the smoothness of all functions, we observe that

$$
\operatorname{div}\left(u P_{1, k}\right)+\left(\frac{v_{k}}{|u|}-1\right) u \cdot \nabla P_{1, k}=\frac{v_{k}}{|u|} u \cdot \nabla P_{1, k} .
$$


Thus, by using (39) and (43), we compute, for any $0<r<\infty$,

$$
\begin{aligned}
(I I I) & \leqslant C \cdot\left\|\frac{v_{k}}{|u|} u \cdot \nabla P_{1, k}\right\|_{L^{1}\left(Q_{k-1}\right)} \leqslant C\left\|\left|v_{k}\right| \cdot\left|\nabla P_{1, k}\right|\right\|_{L^{1}\left(Q_{k-1}\right)} \\
& \leqslant\left\|v_{k}\right\|_{L^{2}\left(T_{k-1}, 0 ; L^{1}\left(B_{k-1}\right)\right)} \cdot\left\|\nabla P_{1, k}\right\|_{L^{2}\left(T_{k-1}, 0 ; L^{\infty}\left(B_{k-1}\right)\right)} \\
& \leqslant\left\|\mathbf{1}_{v_{k}>0}\right\|_{L^{2}\left(T_{k-1}, 0 ; L^{2}\left(B_{k-1}\right)\right)}\left\|v_{k}\right\|_{L^{\infty}\left(T_{k-1}, 0 ; L^{2}\left(B_{k-1}\right)\right)} \cdot 2^{12 k} \\
& \leqslant C 2^{\frac{43 k}{3}} U_{k-1}^{\frac{5}{6}} U_{k-1}^{\frac{1}{2}} \leqslant C 2^{\frac{43 k}{3}} U_{k-1}^{\frac{4}{3}} .
\end{aligned}
$$

For (IV) (local pressure term), as we did for (III), observe

$$
\operatorname{div}\left(u P_{2, k}\right)+\left(\frac{v_{k}}{|u|}-1\right) u \cdot \nabla P_{2, k}=\frac{v_{k}}{|u|} u \cdot \nabla P_{2, k} .
$$

By the definition of $P_{2, k}$, we have

$$
\begin{aligned}
-\Delta P_{2, k} & =\sum_{i j} \partial_{i} \partial_{j}\left(\psi_{k} w_{i} u_{j}\right)=\sum_{i j} \partial_{i}\left(\left(\partial_{j} \psi_{k}\right) w_{i} u_{j}+\psi_{k}\left(\partial_{j} w_{i}\right) u_{j}\right) \\
& =\sum_{i j} \partial_{i}\left(\left(\partial_{j} \psi_{k}\right) w_{i} u_{j}\left(1-\frac{v_{k}}{|u|}\right)+\left(\partial_{j} \psi_{k}\right) w_{i} u_{j} \frac{v_{k}}{|u|}+\psi_{k}\left(\partial_{j} w_{i}\right) u_{j}\left(1-\frac{v_{k}}{|u|}\right)+\psi_{k}\left(\partial_{j} w_{i}\right) u_{j} \frac{v_{k}}{|u|}\right)
\end{aligned}
$$

and

$$
\begin{aligned}
-\Delta\left(\nabla P_{2, k}\right)= & \sum_{i j} \partial_{i} \nabla\left(\left(\partial_{j} \psi_{k}\right) w_{i} u_{j}\left(1-\frac{v_{k}}{|u|}\right)+\left(\partial_{j} \psi_{k}\right) w_{i} u_{j} \frac{v_{k}}{|u|}\right. \\
& \left.+\psi_{k}\left(\partial_{j} w_{i}\right) u_{j}\left(1-\frac{v_{k}}{|u|}\right)+\psi_{k}\left(\partial_{j} w_{i}\right) u_{j} \frac{v_{k}}{|u|}\right) .
\end{aligned}
$$

Thus we can decompose $\nabla P_{2, k}$ by the Riesz transform into

$$
\nabla P_{2, k}=G_{1, k}+G_{2, k}+G_{3, k}+G_{4, k}
$$

where

$$
\begin{aligned}
G_{1, k} & =\sum_{i j}\left(\partial_{i} \nabla\right)(-\Delta)^{-1}\left(\left(\partial_{j} \psi_{k}\right) w_{i} u_{j}\left(1-\frac{v_{k}}{|u|}\right)\right), \\
G_{2, k} & =\sum_{i j}\left(\partial_{i} \nabla\right)(-\Delta)^{-1}\left(\left(\partial_{j} \psi_{k}\right) w_{i} u_{j} \frac{v_{k}}{|u|}\right) \\
G_{3, k} & =\sum_{i j}\left(\partial_{i} \nabla\right)(-\Delta)^{-1}\left(\psi_{k}\left(\partial_{j} w_{i}\right) u_{j}\left(1-\frac{v_{k}}{|u|}\right)\right) \quad \text { and } \\
G_{4, k} & =\sum_{i j}\left(\partial_{i} \nabla\right)(-\Delta)^{-1}\left(\psi_{k}\left(\partial_{j} w_{i}\right) u_{j} \frac{v_{k}}{|u|}\right) .
\end{aligned}
$$

From $L^{p}$-boundedness of the Riesz transform with the fact $\operatorname{supp}\left(\psi_{k}\right) \subset B_{k-(5 / 6)} \subset B_{k-1}$, we have

$$
\begin{aligned}
& \left\|G_{2, k}\right\|_{L^{2}\left(T_{k-1}, 0 ; L^{2}\left(\mathbb{R}^{3}\right)\right)} \leqslant C 2^{3 k}\|w\|_{L^{2}\left(T_{k-1}, 0 ; L^{\infty}\left(B_{k-1}\right)\right)} \cdot\left\|v_{k}\right\|_{L^{\infty}\left(T_{k-1}, 0 ; L^{2}\left(B_{k-1}\right)\right)}, \\
& \left\|G_{4, k}\right\|_{L^{2}\left(T_{k-1}, 0 ; L^{2}\left(\mathbb{R}^{3}\right)\right)} \leqslant C \cdot\|\nabla w\|_{L^{2}\left(T_{k-1}, 0 ; L^{\infty}\left(B_{k-1}\right)\right)} \cdot\left\|v_{k}\right\|_{L^{\infty}\left(T_{k-1}, 0 ; L^{2}\left(B_{k-1}\right)\right)} .
\end{aligned}
$$

Similarly, we have, for any $1<p<\infty$,

$$
\begin{aligned}
& \left\|G_{1, k}\right\|_{L^{2}\left(T_{k-1}, 0 ; L^{p}\left(\mathbb{R}^{3}\right)\right)} \leqslant C_{p} \cdot 2^{3 k}\|w\|_{L^{2}\left(T_{k-1}, 0 ; L^{\infty}\left(B_{k-1}\right)\right)} \quad \text { and } \\
& \left\|G_{3, k}\right\|_{L^{2}\left(T_{k-1}, 0 ; L^{p}\left(\mathbb{R}^{3}\right)\right)} \leqslant C_{p} \cdot\|\nabla w\|_{L^{2}\left(T_{k-1}, 0 ; L^{\infty}\left(B_{k-1}\right)\right)} .
\end{aligned}
$$

Therefore, by using (33) and (35), we get 


$$
\left\|\left|G_{2, k}\right|+\left|G_{4, k}\right|\right\|_{L^{2}\left(T_{k-1}, 0 ; L^{2}\left(\mathbb{R}^{3}\right)\right)} \leqslant \begin{cases}C \cdot 2^{3 k} \cdot U_{k-1}^{\frac{1}{2}}, & \text { if } r \geqslant s_{1}, \\ C \cdot 2^{3 k} \cdot \frac{1}{r^{3}} \cdot U_{k-1}^{\frac{1}{2}}, & \text { if } r<s_{1},\end{cases}
$$

and, for any $1<p<\infty$,

$$
\left\|\left|G_{1, k}\right|+\left|G_{3, k}\right|\right\|_{L^{2}\left(T_{k-1}, 0 ; L^{p}\left(\mathbb{R}^{3}\right)\right)} \leqslant \begin{cases}C_{p} \cdot 2^{3 k}, & \text { if } r \geqslant s_{1}, \\ C_{p} \cdot 2^{3 k} \cdot \frac{1}{r^{3}}, & \text { if } r<s_{1} .\end{cases}
$$

Thus, by using the above estimates and (43), for $r \geqslant s_{1}$ and $p>5$, we compute

$$
\begin{aligned}
(I V) \leqslant & C \cdot\left\|\frac{v_{k}}{|u|} u \cdot \nabla P_{2, k}\right\|_{L^{1}\left(Q_{k-1}\right)} \leqslant C\left\|\left|v_{k}\right| \cdot\left|\nabla P_{2, k}\right|\right\|_{L^{1}\left(Q_{k-1}\right)} \\
\leqslant & C\left\|\left|v_{k}\right| \cdot\left(\left|G_{1, k}\right|+\left|G_{3, k}\right|\right)\right\|_{L^{1}\left(Q_{k-1}\right)}+C\left\|\left|v_{k}\right| \cdot\left(\left|G_{2, k}\right|+\left|G_{4, k}\right|\right)\right\|_{L^{1}\left(Q_{k-1}\right)} \\
\leqslant & \left\|v_{k}\right\|_{L^{2}\left(T_{k-1}, 0 ; L^{\frac{p}{p-1}}\left(B_{k-1}\right)\right)} \cdot\left\|\left|G_{1, k}\right|+\left|G_{3, k}\right|\right\|_{L^{2}\left(T_{k-1}, 0 ; L^{p}\left(B_{k-1}\right)\right)} \\
& +\left\|v_{k}\right\|_{L^{2}\left(T_{k-1}, 0 ; L^{2}\left(B_{k-1}\right)\right)} \cdot\left\|\left|G_{2, k}\right|+\left|G_{4, k}\right|\right\|_{L^{2}\left(T_{k-1}, 0 ; L^{2}\left(B_{k-1}\right)\right)} \\
\leqslant & C \cdot C_{p} \cdot 2^{\frac{16 k}{3}} U_{k-1}^{\frac{4 p-5}{3 p}} .
\end{aligned}
$$

By the same way, for $r<s_{1}$ and $p>5$, we obtain

$$
(I V) \leqslant C \cdot C_{p} \cdot \frac{1}{r^{3}} 2^{\frac{16 k}{3}} U_{k-1}^{\frac{4 p-5}{3 p}} .
$$

Thus, by taking $p=10$, we obtain

$$
(I V) \leqslant \begin{cases}C \cdot 2^{\frac{16 k}{3}} U_{k-1}^{\frac{7}{6}}, & \text { if } r \geqslant s_{1}, \\ C \cdot \frac{1}{r^{3}} 2^{\frac{16 k}{3}} U_{k-1}^{\frac{7}{6}}, & \text { if } r<s_{1} .\end{cases}
$$

Finally, combining (50), (51), (52), (53) and (54) gives us

$$
(I)+(I I)+(I I I)+(I V) \leqslant \begin{cases}C^{k} \cdot U_{k-1}^{\frac{7}{6}}, & \text { if } r \geqslant s_{1}, \\ \frac{1}{r^{3}} \cdot C^{k} \cdot U_{k-1}^{\frac{7}{6}}, & \text { if } r<s_{1} .\end{cases}
$$

\subsection{De Giorgi argument to get a control for small $r$}

The next result handles the case of small $r$ including the case $r=0$.

Recall the definition of $s_{k}$ in (24) first. It is the distance between $B_{k-1}^{C}$ and $B_{k-\frac{5}{6}}$, and $s_{k}$ is strictly decreasing to zero as $k \rightarrow \infty$. For any $0<r<s_{1}$ we define $k_{r}$ as the integer such that $s_{k_{r}+1}<r \leqslant s_{k_{r}}$. Note that $k_{r} \geqslant 1$, and it is increasing to $\infty$ as $r$ goes to zero. For the case $r=0$, we simply define $k_{r}=k_{0}=\infty$.

Lemma 3.5. There exist constants $\delta_{2}>0$ and $\bar{C}_{2}>1$ such that if $u$ is a solution of (Problem II- $r$ ) for some $0 \leqslant r<s_{1}$ verifying

$$
\|u\|_{L^{\infty}\left(-2,0 ; L^{2}\left(B\left(\frac{5}{4}\right)\right)\right)}+\|P\|_{L^{1}\left(-2,0 ; L^{1}(B(1))\right)}+\|\nabla u\|_{L^{2}\left(-2,0 ; L^{2}\left(B\left(\frac{5}{4}\right)\right)\right)} \leqslant \delta_{2}
$$

then we have

$$
U_{k} \leqslant\left(\bar{C}_{2}\right)^{k} U_{k-1}^{\frac{7}{6}} \quad \text { for any integer } k \text { such that } 1 \leqslant k \leqslant k_{r} .
$$

Remark 3.7. Note that $\delta_{2}$ and $\bar{C}_{2}$ are independent of any $r \in\left[0, s_{1}\right)$, and the exponent $7 / 6$ is not optimal. 
Remark 3.8. This lemma says that even though $r$ is very small, we can prove the above uniform estimate for the first few steps $k \leqslant k_{r}$. Moreover, the number $k_{r}$ of these steps is increasing to the infinity with a certain rate as $r$ goes to zero. In Section 3.4, we will see that this rate is enough to obtain a uniform estimate for any small $r$ once we combine the two Lemmas 3.4 and 3.5.

Proof of Lemma 3.5. In this proof, we can borrow any estimates in the proof of the previous Lemma 3.4 except those which depend on $r$ and blow up as $r$ goes to zero (recall that every estimate depending on $r$ in the previous Lemma 3.4 was obtained when and only when we used the smallness condition of $L^{2}$-norm of $\mathcal{M}(|\nabla u|)$, which we do not assume in Lemma 3.5).

Let $0 \leqslant r<s_{1}$. We take any integer $k$ such that $1 \leqslant k \leqslant k_{r}$. As we chose $\delta_{1}$ in the previous Lemma 3.4, we assume $\delta_{2}>0$ first so small that

$$
\delta_{2}<1, \quad 10 \Lambda_{1} \Lambda_{2} \delta_{2} \leqslant \frac{1}{2} .
$$

We begin this proof by decomposing $w^{\prime}$ by

$$
w^{\prime}=u * \phi_{r}=\left(u\left(1-\frac{v_{k}}{|u|}\right)\right) * \phi_{r}+\left(u \frac{v_{k}}{|u|}\right) * \phi_{r}=w^{\prime, 1}+w^{\prime, 2} .
$$

Thus the convective velocity $w$ has a new decomposition: $w=w^{\prime}-w^{\prime \prime}=\left(w^{\prime, 1}+w^{\prime, 2}\right)-w^{\prime \prime}=\left(w^{\prime, 1}-w^{\prime \prime}\right)+w^{\prime, 2}$. We will verify that $\left(w^{\prime, 1}-w^{\prime \prime}\right)$ is bounded, and $w^{\prime, 2}$ can be controlled locally. First, for $w^{\prime, 1}$,

$$
\left|w^{\prime, 1}(t, x)\right|=\left|\left(\left(u\left(1-\frac{v_{k}}{|u|}\right)\right) * \phi_{r}\right)(t, x)\right| \leqslant\left\|u\left(1-\frac{v_{k}}{|u|}\right)(t, \cdot)\right\|_{L^{\infty}\left(\mathbb{R}^{3}\right)} \leqslant 1
$$

for any $-4 \leqslant t$ and any $x \in \mathbb{R}^{3}$. From (36), we still have

$$
\left\|w^{\prime \prime}\right\|_{L^{\infty}\left(-2,0 ; L^{\infty}(B(2))\right)} \leqslant C \bar{\delta} \leqslant C .
$$

Combining above two results,

$$
\left\|\left|w^{\prime, 1}\right|+\left|w^{\prime \prime}\right|\right\|_{L^{\infty}\left(-2,0 ; L^{\infty}(B(2))\right)} \leqslant C .
$$

For $w^{\prime, 2}$, we observe that any $L^{p}$-norm of $w^{\prime, 2}=\left(u \frac{v_{k}}{|u|}\right) * \phi_{r}$ in $B_{k-\frac{5}{6}}$ is less than or equal to that of $v_{k}$ in $B_{k-1}$ because $r \leqslant s_{k_{r}} \leqslant s_{k}$ and $s_{k}$ is the distance between $B_{k-1}^{C}$ and $B_{k-\frac{5}{6}}$ (see (8)). Thus we have, for any $1 \leqslant p \leqslant \infty$,

$$
\begin{aligned}
\left\|w^{\prime, 2}\right\|_{L^{p}\left(T_{k-1}, 0 ; L^{p}\left(B_{k-\frac{5}{6}}\right)\right)} & =\left\|\left(u \frac{v_{k}}{|u|}\right) * \phi_{r}\right\|_{L^{p}\left(T_{k-1}, 0 ; L^{p}\left(B_{k-\frac{5}{6}}\right)\right)} \\
& =\left\|\left|v_{k}\right| * \phi_{r}\right\|_{L^{p}\left(T_{k-1}, 0 ; L^{p}\left(B_{\left.k-\frac{5}{6}\right)}\right)\right.} \leqslant\left\|v_{k}\right\|_{L^{p}\left(Q_{k-1}\right)} .
\end{aligned}
$$

So, by using (43), we have

$$
\left\|w^{\prime, 2}\right\|_{L^{p}\left(T_{k-1}, 0 ; L^{p}\left(B_{k-\frac{5}{6}}\right)\right)} \leqslant C 2^{\frac{7 k}{3}} U_{k-1}^{\frac{5}{3 p}}, \quad \text { for any } 1 \leqslant p \leqslant \frac{10}{3} .
$$

Remark 3.9. The above computations show that, for $0 \leqslant r<s_{1}$ and for $1 \leqslant k \leqslant k_{r}$, the convective velocity $w$ can be decomposed into one bounded part $\left(w^{\prime, 1}-w^{\prime \prime}\right)$ and the other part $w^{\prime, 2}$, which has a certain contribution to the power of $U_{k-1}$.

Recall that the transport term estimate (31) is valid for $0<r \leqslant s_{1}$ without having the smallness condition of $\|\mathcal{M}(|\nabla u|)\|_{L^{2}}$ (see (37)). Moreover, the argument in (37) says that (31) holds even for the case $r=0$. As a result, for any $r \in\left[0, s_{1}\right.$ ), we have the same pressure estimates (39), (40) and (41). Thus we can follow the proof of the previous Lemma 3.4 up to (48) without any single modification. It remains to control $(I)-(I V)$.

For $(I)$, the estimate (50) holds because (50) is independent of $r$. 
For (II), by using (57) and (59) with the fact $\operatorname{supp}\left(\eta_{k}\right) \subset B_{k-\frac{1}{3}} \subset B_{k-\frac{5}{6}}$, we have

$$
\begin{aligned}
(I I) & =\left\|\left|\nabla \eta_{k}\right| \cdot|w| \cdot\left|v_{k}\right|^{2}\right\|_{L^{1}\left(Q_{k-1}\right)} \\
& \leqslant C 2^{3 k}\left(\left\|\left(\left|w^{\prime, 1}\right|+\left|w^{\prime \prime}\right|\right) \cdot\left|v_{k}\right|^{2}\right\|_{L^{1}\left(Q_{k-1}\right)}+\left\|\left|w^{\prime, 2}\right| \cdot\left|v_{k}\right|^{2}\right\|_{L^{1}\left(T_{k-1}, 0 ; L^{1}\left(B_{k-\frac{5}{6}}\right)\right)}\right) \\
& \leqslant C 2^{3 k}\left\|v_{k}\right\|_{L^{2}\left(Q_{k-1}\right)}^{2}+C 2^{3 k}\left\|w^{\prime, 2}\right\|_{L^{\frac{10}{3}}\left(T_{k-1}, 0 ; L^{\frac{10}{3}}\left(B_{k-\frac{5}{6}}\right)\right)} \cdot\left\|\left|v_{k}\right|^{2}\right\|_{L^{\frac{10}{7}}\left(Q_{k-\frac{5}{6}}\right)} \\
& \leqslant C 2^{\frac{23 k}{3}} U_{k-1}^{\frac{5}{3}}+C 2^{10 k} U_{k-1}^{\frac{5}{3}} \leqslant C 2^{10 k} U_{k-1}^{\frac{5}{3}} .
\end{aligned}
$$

For (III) (non-local pressure term), we have (53) since (53) is independent of $r$.

For (IV) (local pressure term), by definition of $P_{2, k}$ and decomposition of $w$,

$$
\begin{aligned}
-\Delta P_{2, k}= & \sum_{i j} \partial_{i} \partial_{j}\left(\psi_{k} w_{i} u_{j}\left(1-\frac{v_{k}}{|u|}\right)+\psi_{k} w_{i} u_{j} \frac{v_{k}}{|u|}\right) \\
= & \sum_{i j} \partial_{i} \partial_{j}\left(\psi_{k}\left(w_{i}^{\prime, 1}-w_{i}^{\prime \prime}\right) u_{j}\left(1-\frac{v_{k}}{|u|}\right)+\psi_{k} w_{i}^{\prime, 2} u_{j}\left(1-\frac{v_{k}}{|u|}\right)\right. \\
& \left.+\psi_{k}\left(w_{i}^{\prime, 1}-w_{i}^{\prime \prime}\right) u_{j} \frac{v_{k}}{|u|}+\psi_{k} w_{i}^{\prime 2} u_{j} \frac{v_{k}}{|u|}\right) .
\end{aligned}
$$

Thus we can decompose $P_{2, k}$ by

$$
P_{2, k}=P_{2, k, 1}+P_{2, k, 2}+P_{2, k, 3}+P_{2, k, 4}
$$

where

$$
\begin{aligned}
& P_{2, k, 1}=\sum_{i j}\left(\partial_{i} \partial_{j}\right)(-\Delta)^{-1}\left(\psi_{k}\left(w_{i}^{\prime, 1}-w_{i}^{\prime \prime}\right) u_{j}\left(1-\frac{v_{k}}{|u|}\right)\right), \\
& P_{2, k, 2}=\sum_{i j}\left(\partial_{i} \partial_{j}\right)(-\Delta)^{-1}\left(\psi_{k} w_{i}^{\prime, 2} u_{j}\left(1-\frac{v_{k}}{|u|}\right)\right), \\
& P_{2, k, 3}=\sum_{i j}\left(\partial_{i} \partial_{j}\right)(-\Delta)^{-1}\left(\psi_{k}\left(w_{i}^{\prime, 1}-w_{i}^{\prime \prime}\right) u_{j} \frac{v_{k}}{|u|}\right) \quad \text { and } \\
& P_{2, k, 4}=\sum_{i j}\left(\partial_{i} \partial_{j}\right)(-\Delta)^{-1}\left(\psi_{k} w_{i}^{\prime, 2} u_{j} \frac{v_{k}}{|u|}\right) .
\end{aligned}
$$

By using $\left|u\left(1-\frac{v_{k}}{|u|}\right)\right| \leqslant 1$ and the fact that $\psi_{k}$ is supported in $B_{k-\frac{5}{6}}$, we have

$$
\begin{aligned}
\left\|P_{2, k, 1}\right\|_{L^{p}\left(T_{k-1}, 0 ; L^{p}\left(\mathbb{R}^{3}\right)\right)} & \leqslant C_{p} \quad \text { for } 1<p<\infty, \quad \text { and } \\
\left\|P_{2, k, 2}\right\|_{L^{p}\left(T_{k-1}, 0 ; L^{p}\left(\mathbb{R}^{3}\right)\right)} & \leqslant C_{p}\left\|\left|\psi_{k}\right| \cdot\left|w^{\prime, 2}\right|\right\|_{L^{p}\left(T_{k-1}, 0 ; L^{p}\left(\mathbb{R}^{3}\right)\right)} \\
& \leqslant C C_{p} 2^{\frac{7 k}{3}} U_{k-1}^{\frac{5}{3 p}} \quad \text { for } 1 \leqslant p \leqslant \frac{10}{3},
\end{aligned}
$$

thanks to (57) and (59). Observe that for $i=1,2$,

$$
\operatorname{div}\left(u G_{i}\right)+\left(\frac{v_{k}}{|u|}-1\right) u \cdot \nabla G_{i}=\operatorname{div}\left(v_{k} \frac{u}{|u|} G_{i}\right)-G_{i} \operatorname{div}\left(\frac{u v_{k}}{|u|}\right) .
$$

For $P_{2, k, 1}$, by using (42), (43), (45), (63) and (61) with $p=10$, we have 


$$
\begin{aligned}
& \int_{T_{k-1}}^{0}\left|\int_{\mathbb{R}^{3}} \eta_{k}(x)\left(\operatorname{div}\left(u P_{2, k, 1}\right)+\left(\frac{v_{k}}{|u|}-1\right) u \cdot \nabla P_{2, k, 1}\right)(s, x) d x\right| d s \\
& \quad \leqslant C^{3 k}\left\|v_{k} \cdot\left|P_{2, k, 1}\right|\right\|_{L^{1}\left(Q_{k-1}\right)}+3\left\|d_{k} \cdot\left|P_{2, k, 1}\right|\right\|_{L^{1}\left(Q_{k-1}\right)} \\
& \quad \leqslant C^{3 k}\left\|v_{k}\right\|_{L^{\frac{10}{9}}\left(Q_{k-1}\right)} \cdot\left\|P_{2, k, 1}\right\|_{L^{10}\left(Q_{k-1}\right)}+3\left\|d_{k}\right\|_{L^{\frac{10}{9}}\left(Q_{k-1}\right)} \cdot\left\|P_{2, k, 1}\right\|_{L^{10}\left(Q_{k-1}\right)} \\
& \quad \leqslant C 2^{\frac{16 k}{3}} U_{k-1}^{\frac{3}{2}}+C 2^{\frac{5 k}{3}} U_{k-1}^{\frac{7}{6}} \leqslant C 2^{\frac{16 k}{3}} U_{k-1}^{\frac{7}{6}} .
\end{aligned}
$$

Likewise, for $P_{2, k, 2}$, by using (62) instead of (61), we have

$$
\begin{aligned}
& \int_{T_{k-1}}^{0}\left|\int_{\mathbb{R}^{3}} \eta_{k}(x)\left(\operatorname{div}\left(u P_{2, k, 2}\right)+\left(\frac{v_{k}}{|u|}-1\right) u \cdot \nabla P_{2, k, 2}\right)(s, x) d x\right| d s \\
& \quad \leqslant C 2^{\frac{23 k}{3}} U_{k-1}^{\frac{5}{3}}+C 2^{4 k} U_{k-1}^{\frac{4}{3}} \leqslant C 2^{\frac{23 k}{3}} U_{k-1}^{\frac{4}{3}} .
\end{aligned}
$$

From the definitions of $P_{2, k, 3}$ and $P_{2, k, 4}$ with $\operatorname{div}(w)=0$, we have

$$
\begin{aligned}
-\Delta \nabla\left(P_{2, k, 3}+P_{2, k, 4}\right) & =\sum_{i j} \partial_{i} \partial_{j} \nabla\left(\psi_{k} w_{i} u_{j} \frac{v_{k}}{|u|}\right) \\
& =\sum_{i j} \nabla \partial_{j}\left(\left(\partial_{i} \psi_{k}\right) w_{i} u_{j} \frac{v_{k}}{|u|}+\psi_{k} w_{i} \partial_{i}\left(u_{j} \frac{v_{k}}{|u|}\right)\right) .
\end{aligned}
$$

Then we use the fact $w=\left(w^{\prime, 1}-w^{\prime \prime}\right)+w^{\prime, 2}$ so that we can decompose

$$
\nabla\left(P_{2, k, 3}+P_{2, k, 4}\right)=H_{1, k}+H_{2, k}+H_{3, k}+H_{4, k}
$$

where

$$
\begin{aligned}
& H_{1, k}=\sum_{i j}\left(\nabla \partial_{j}\right)(-\Delta)^{-1}\left(\left(\partial_{i} \psi_{k}\right)\left(w_{i}^{\prime, 1}-w_{i}^{\prime \prime}\right) u_{j} \frac{v_{k}}{|u|}\right), \\
& H_{2, k}=\sum_{i j}\left(\nabla \partial_{j}\right)(-\Delta)^{-1}\left(\left(\partial_{i} \psi_{k}\right) w_{i}^{\prime, 2} u_{j} \frac{v_{k}}{|u|}\right), \\
& H_{3, k}=\sum_{i j}\left(\nabla \partial_{j}\right)(-\Delta)^{-1}\left(\psi_{k}\left(w_{i}^{\prime 1}-w_{i}^{\prime \prime}\right) \partial_{i}\left(u_{j} \frac{v_{k}}{|u|}\right)\right) \quad \text { and } \\
& H_{4, k}=\sum_{i j}\left(\nabla \partial_{j}\right)(-\Delta)^{-1}\left(\psi_{k} w_{i}^{\prime 2} \partial_{i}\left(u_{j} \frac{v_{k}}{|u|}\right)\right) .
\end{aligned}
$$

By using $|u| \leqslant 1+v_{k}$, we have

$$
\begin{aligned}
& \int_{T_{k-1}}^{0}\left|\int_{\mathbb{R}^{3}} \eta_{k}(x)\left(\operatorname{div}\left(u\left(P_{2, k, 3}+P_{2, k, 4}\right)\right)+\left(\frac{v_{k}}{|u|}-1\right) u \cdot \nabla\left(P_{2, k, 3}+P_{2, k, 4}\right)\right) d x\right| d s \\
& \leqslant C^{3 k} \int_{Q_{k-1}}\left(1+v_{k}\right) \cdot\left|\left(P_{2, k, 3}+P_{2, k, 4}\right)(s, x)\right|+\left|\nabla\left(P_{2, k, 3}+P_{2, k, 4}\right)\right| d x d s \\
& \quad \leqslant C^{3 k}\left(\left\|P_{2, k, 3}\right\|_{L^{1}\left(Q_{k-1}\right)}+\left\|v_{k} \cdot\left|P_{2, k, 3}\right|\right\|_{L^{1}\left(Q_{k-1}\right)}+\left\|P_{2, k, 4}\right\|_{L^{1}\left(Q_{k-1}\right)}+\left\|v_{k} \cdot\left|P_{2, k, 4}\right|\right\|_{L^{1}\left(Q_{k-1}\right)}\right. \\
& \left.\quad+\left\|H_{1, k}\right\|_{L^{1}\left(Q_{k-1}\right)}+\left\|H_{2, k}\right\|_{L^{1}\left(Q_{k-1}\right)}+\left\|H_{3, k}\right\|_{L^{1}\left(Q_{k-1}\right)}+\left\|H_{4, k}\right\|_{L^{1}\left(Q_{k-1}\right)}\right) .
\end{aligned}
$$

From (43) and (57) with the Riesz transform, we obtain 


$$
\begin{aligned}
& \left\|P_{2, k, 3}\right\|_{L^{1}\left(Q_{k-1}\right)} \leqslant C\left\|P_{2, k, 3}\right\|_{L^{\frac{10}{9}}\left(T_{k-1}, 0 ; L^{\frac{10}{9}}\left(\mathbb{R}^{3}\right)\right)} \leqslant C\left\|v_{k}\right\|_{L^{\frac{10}{9}}\left(Q_{k-1}\right)} \leqslant C 2^{\frac{7 k}{3}} U_{k-1}^{\frac{3}{2}}, \\
& \left\|H_{1, k}\right\|_{L^{1}\left(Q_{k-1}\right)} \leqslant C 2^{\frac{16 k}{3}} U_{k-1}^{\frac{3}{2}}, \quad \text { and } \\
& \left\|v_{k} \cdot \mid P_{2, k, 3}\right\|_{L^{1}\left(Q_{k-1}\right)} \leqslant\left\|v_{k}\right\|_{L^{2}\left(Q_{k-1}\right)}\left\|P_{2, k, 3}\right\|_{L^{2}\left(Q_{k-1}\right)} \\
& \leqslant C 2^{\frac{7 k}{3}} U_{k-1}^{\frac{5}{6}} \cdot C 2^{\frac{7 k}{3}} U_{k-1}^{\frac{5}{6}} \leqslant C 2^{\frac{14 k}{3}} U_{k-1}^{\frac{5}{3}} .
\end{aligned}
$$

Using (43), (59), (42) and (45), we have

$$
\begin{aligned}
& \left\|P_{2, k, 4}\right\|_{L^{1}\left(Q_{k-1}\right)} \leqslant C 2^{\frac{14 k}{3}} U_{k-1}^{\frac{3}{2}}, \\
& \left\|H_{2, k}\right\|_{L^{1}\left(Q_{k-1}\right)} \leqslant C 2^{\frac{23 k}{3}} U_{k-1}^{\frac{3}{2}}, \\
& \left\|v_{k} \cdot\left|P_{2, k, 4}\right|\right\|_{L^{1}\left(Q_{k-1}\right)} \leqslant C 2^{\frac{21 k}{3}} U_{k-1}^{\frac{5}{3}}, \\
& \left\|H_{3, k}\right\|_{L^{1}\left(Q_{k-1}\right)} \leqslant C 2^{\frac{5 k}{3}} U_{k-1}^{\frac{7}{6}}, \quad \text { and } \\
& \left\|H_{4, k}\right\|_{L^{1}\left(Q_{k-1}\right)} \leqslant C 2^{4 k} U_{k-1}^{\frac{7}{6}} .
\end{aligned}
$$

Combining (64), (65) and (66) together with (67)-(74), we obtain

$$
(I V) \leqslant C 2^{\frac{23 k}{3}} U_{k-1}^{\frac{7}{6}} \text {. }
$$

Finally we combine (60) and (75) together with (50) and (53) in the previous Lemma 3.4 in order to finish the proof of Lemma 3.5.

\subsection{Combining the two De Giorgi arguments}

First we present the following lemma. Then the proof of Proposition 2.1 will follow. The following lemma says that certain non-linear estimates give the zero limit if the initial term is sufficiently small. This fact is one of the key arguments of the De Giorgi method.

Lemma 3.6. Let $C>1$ and $\beta>1$. Then there exists a constant $C_{0}^{*}$ such that for every sequence verifying both $0 \leqslant W_{0}<C_{0}^{*}$ and

$$
0 \leqslant W_{k} \leqslant C^{k} \cdot W_{k-1}^{\beta} \text { for any } k \geqslant 1,
$$

we have $\lim _{k \rightarrow \infty} W_{k}=0$.

Proof. It is quite standard, or see Lemma 1 in [42].

Finally we are ready to prove Proposition 2.1.

Proof of Proposition 2.1. Suppose that $u$ is a solution of (Problem II- $r$ ) for some $0 \leqslant r<\infty$ verifying

$$
\begin{aligned}
& \|u\|_{L^{\infty}\left(-2,0 ; L^{2}\left(B\left(\frac{5}{4}\right)\right)\right)}+\|P\|_{L^{1}\left(-2,0 ; L^{1}(B(1))\right)}+\|\nabla u\|_{L^{2}\left(-2,0 ; L^{2}\left(B\left(\frac{5}{4}\right)\right)\right)} \leqslant \delta \quad \text { and } \\
& \|\mathcal{M}(|\nabla u|)\|_{L^{2}\left(-4,0 ; L^{2}(B(2))\right)} \leqslant \delta
\end{aligned}
$$

where $\delta$ will be chosen within the proof.

From Lemmas 3.4 and 3.5, by assuming $\delta \leqslant \min \left(\delta_{1}, \delta_{2}\right)$, we have

$$
U_{k} \leqslant \begin{cases}\left(\bar{C}_{1}\right)^{k} U_{k-1}^{\frac{7}{6}}, & \text { for any } k \geqslant 1 \text { if } r \geqslant s_{1}, \\ \frac{1}{r^{3}} \cdot\left(\bar{C}_{1}\right)^{k} U_{k-1}^{\frac{7}{6}}, & \text { for any } k \geqslant 1 \text { if } 0<r<s_{1}, \\ \left(\bar{C}_{2}\right)^{k} U_{k-1}^{\frac{7}{6}}, & \text { for } k=1,2, \ldots, k_{r} \text { if } 0 \leqslant r<s_{1} .\end{cases}
$$


Note that $k_{r}=\infty$ if $r=0$. Thus we can combine the case $r=0$ with the case $r \geqslant s_{1}$ into one estimate:

$$
U_{k} \leqslant\left(\bar{C}_{3}\right)^{k} U_{k-1}^{\frac{7}{6}} \text { for any } k \geqslant 1 \text { if either } r \geqslant s_{1} \text { or } r=0,
$$

where we define $\bar{C}_{3}=\max \left(\bar{C}_{1}, \bar{C}_{2}\right)$.

We consider now the case $0<r<s_{1}$. Recall that $s_{k}=D \cdot 2^{-3 k}$ where $D=((\sqrt{2}-1) 2 \sqrt{2})>1$ and $s_{k_{r}+1}<r \leqslant s_{k_{r}}$ for any $r \in\left(0, s_{1}\right)$. It gives us $r \geqslant D \cdot 2^{-3\left(k_{r}+1\right)}$. Thus if $k \geqslant k_{r}$ and if $0<r<s_{1}$, then the second line in (76) becomes

$$
\begin{aligned}
U_{k} & \leqslant \frac{1}{r^{3}} \cdot\left(\bar{C}_{1}\right)^{k} U_{k-1}^{\frac{7}{6}} \leqslant \frac{2^{9\left(k_{r}+1\right)}}{D^{3}} \cdot\left(\bar{C}_{1}\right)^{k} U_{k-1}^{\frac{7}{6}} \\
& \leqslant 2^{9(k+1)} \cdot\left(\bar{C}_{1}\right)^{k} U_{k-1}^{\frac{7}{6}} \leqslant\left(2^{18} \cdot \bar{C}_{1}\right)^{k} U_{k-1}^{\frac{7}{6}} .
\end{aligned}
$$

So we have for any $r \in\left(0, s_{1}\right)$,

$$
U_{k} \leqslant \begin{cases}\left(2^{18} \cdot \bar{C}_{1}\right)^{k} U_{k-1}^{\frac{7}{6}}, & \text { for any } k \geqslant k_{r}, \\ \left(\bar{C}_{2}\right)^{k} U_{k-1}^{\frac{7}{6}}, & \text { for } k=1,2, \ldots, k_{r} .\end{cases}
$$

Define $\bar{C}=\max \left(2^{18} \cdot \bar{C}_{1}, \bar{C}_{2}, \bar{C}_{3}\right)=\max \left(2^{18} \cdot \bar{C}_{1}, \bar{C}_{2}\right)$. Then we can combine all three cases $r=0,0<r<s_{1}$, and $s_{1} \leqslant r<\infty$ into one uniform estimate:

$$
U_{k} \leqslant(\bar{C})^{k} U_{k-1}^{\frac{7}{6}} \text { for any } k \geqslant 1 \text { and for any } 0 \leqslant r<\infty .
$$

Finally, by using the recursive Lemma 3.6, we obtain $C_{0}^{*}$ such that $U_{k} \rightarrow 0$ as $k \rightarrow 0$ whenever $U_{0}<C_{0}^{*}$. This condition $U_{0}<C_{0}^{*}$ is achievable once we assume $\delta$ so small that $\delta \leqslant \sqrt{\frac{C_{0}^{*}}{2}}$ because

$$
U_{0} \leqslant\left(\|u\|_{L^{\infty}\left(-2,0 ; L^{2}\left(B\left(\frac{5}{4}\right)\right)\right)}+\|P\|_{L^{1}\left(-2,0 ; L^{1}(B(1))\right)}+\|\nabla u\|_{L^{2}\left(-2,0 ; L^{2}\left(B\left(\frac{5}{4}\right)\right)\right)}\right)^{2} .
$$

Thus we fix $\delta=\min \left(\sqrt{\frac{C_{0}^{*}}{2}}, \delta_{1}, \delta_{2}\right)$ which does not depend on any $r \in[0, \infty)$. Observe that for any $k \geqslant 1$,

$$
\sup _{-\frac{3}{2} \leqslant t \leqslant 0} \int_{B\left(\frac{1}{2}\right)}(|u(t, x)|-1)_{+}^{2} d x \leqslant U_{k}
$$

from $E_{k} \leqslant 1$ and $\left(-\frac{3}{2}, 0\right) \times B\left(\frac{1}{2}\right) \subset Q_{k}$. Due to the fact $U_{k} \rightarrow 0$, the conclusion of Proposition 2.1 follows.

\section{Proof of the second local study Proposition 2.2}

First we present technical lemmas, whose proofs will be given in Appendix A. In Section 4.2, it will be explained how to apply the previous local study Proposition 2.1 in order to get an $L^{\infty}$-bound of the velocity $u$. Then, Sections 4.3 and 4.4 will give us $L^{\infty}$-bounds for classical derivatives $\nabla^{d} u$ and for fractional derivatives $(-\Delta)^{\alpha / 2} \nabla^{d} u$, respectively.

\subsection{Some lemmas}

The following lemma present estimates about higher derivatives of pressure which we can find a similar lemma in [41]. However they are different in the sense that here we require $(n-1)$ st order norm of $v_{1}$ to control $n$th derivatives of pressure (see (78)) while, in [41], $n$th order is required. This fact follows the divergence structure and it will be useful for the bootstrap argument in Section 4.3 for large $r$ (we will see (84)). In the following lemmas, $\left(v_{2} \otimes v_{1}\right)$ represents the matrix whose $(i, j)$ component is the product of $j$-th component $v_{2, j}$ of $v_{2}$ and $i$-th one $v_{1, i}$ of $v_{1}$ and $\left(\operatorname{div}\left(v_{2} \otimes v_{1}\right)\right)_{i}=\sum_{j} \partial_{j}\left(v_{2, j} v_{1, i}\right)$.

Lemma 4.1. Suppose that we have $v_{1}, v_{2} \in\left(C^{\infty}(B(1))\right)^{3}$ with $\operatorname{div} v_{1}=\operatorname{div} v_{2}=0$ and $P \in C^{\infty}(B(1))$ which satisfy $-\Delta P=\operatorname{div} \operatorname{div}\left(v_{2} \otimes v_{1}\right)$ 
on $B(1) \subset \mathbb{R}^{3}$. Then, for any $n \geqslant 2,0<b<a<1$ and $1<p<\infty$, we have the two following estimates:

$$
\left\|\nabla^{n} P\right\|_{L^{p}(B(b))} \leqslant C_{(a, b, n, p)}\left(\left\|v_{2}\right\|_{W^{n-1, p_{2}(B(a))}} \cdot\left\|v_{1}\right\|_{W^{n-1, p_{1}(B(a))}}+\|P\|_{L^{1}(B(a))}\right)
$$

where $\frac{1}{p}=\frac{1}{p_{1}}+\frac{1}{p_{2}}$, and

$$
\left\|\nabla^{n} P\right\|_{L^{\infty}(B(b))} \leqslant C_{(a, b, n)}\left(\left\|v_{2}\right\|_{W^{n, \infty}(B(a))} \cdot\left\|v_{1}\right\|_{W^{n, \infty}(B(a))}+\|P\|_{L^{1}(B(a))}\right) .
$$

Note that such constants are independent of any $v_{1}, v_{2}$ and $P$. Also, $\infty$ is allowed for $p_{1}$ and $p_{2}$, e.g. if $p_{1}=\infty$, then $p_{2}=p$.

Proof. See Appendix A.

The following is a local result by using a parabolic regularization. It will be used in Section 4.3 to prove (82) and (84).

Lemma 4.2. Suppose that we have smooth solution $\left(v_{1}, v_{2}, P\right)$ on $Q(1)=(-1,0) \times B(1)$ of

$$
\begin{aligned}
& \partial_{t}\left(v_{1}\right)+\operatorname{div}\left(v_{2} \otimes v_{1}\right)+\nabla P-\Delta v_{1}=0, \\
& \operatorname{div}\left(v_{1}\right)=0 \text { and } \operatorname{div}\left(v_{2}\right)=0 .
\end{aligned}
$$

Then, for any $n \geqslant 1,0<b<a<1,1<p_{1}<\infty$ and $1<p_{2}<\infty$, we have

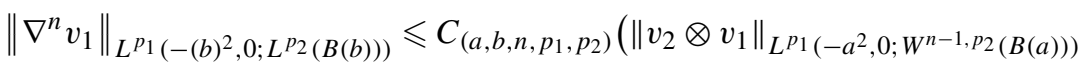

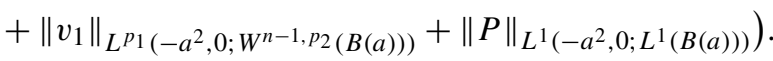

Note that such constants are independent of any $v_{1}, v_{2}$ and $P$.

Proof of Lemma 4.2 is omitted because it is based on the standard parabolic regularization result (e.g. Solonnikov [38]) and precise argument is essentially contained in [41] except that here we consider

$$
\left(v_{1}\right)_{t}+\operatorname{div}\left(v_{2} \otimes v_{1}\right)+\nabla P-\Delta v_{1}=0
$$

while [41] covered

$$
(u)_{t}+\operatorname{div}(u \otimes u)+\nabla P-\Delta u=0 .
$$

The following lemma will be used in Section 4.3, especially when we prove (84) for large $r$.

Lemma 4.3. Suppose that we have smooth solution $\left(v_{1}, v_{2}, P\right)$ on $Q(1)=(-1,0) \times B(1)$ of

$$
\begin{aligned}
& \partial_{t}\left(v_{1}\right)+\left(v_{2} \cdot \nabla\right)\left(v_{1}\right)+\nabla P-\Delta v_{1}=0, \\
& \operatorname{div}\left(v_{1}\right)=0 \quad \text { and } \quad \operatorname{div}\left(v_{2}\right)=0 .
\end{aligned}
$$

Then, for any $n \geqslant 0$ and $0<b<a<1$, we have

$$
\begin{aligned}
\left\|\nabla^{n} v_{1}\right\|_{L^{\infty}\left(-(b)^{2}, 0 ; L^{1}(B(b))\right)} \leqslant & C_{(a, b, n)}\left[\left(\left\|v_{2}\right\|_{L^{2}\left(-a^{2}, 0 ; W^{n, \infty}(B(a))\right)}+1\right) \cdot\left\|v_{1}\right\|_{L^{2}\left(-a^{2}, 0 ; W^{n, 1}(B(a))\right)}\right. \\
& \left.+\left\|\nabla^{n+1} P\right\|_{L^{1}\left(-a^{2}, 0 ; L^{1}(B(a))\right)}\right]
\end{aligned}
$$

and, for any $p \geqslant 1$,

$$
\begin{aligned}
\left\|\nabla^{n} v_{1}\right\|_{L^{\infty}\left(-(b)^{2}, 0 ; L^{p+\frac{1}{2}}(B(b))\right)}^{p+\frac{1}{2}} \leqslant & C_{(a, b, n, p)}\left[\left(\left\|v_{2}\right\|_{L^{2}\left(-a^{2}, 0 ; W^{n, \infty}(B(a))\right)}+1\right) \cdot\left\|v_{1}\right\|_{L^{2}\left(-(a)^{2}, 0 ; W^{n, 2 p}(B(a))\right)}\right. \\
& \left.+\left\|\nabla^{n+1} P\right\|_{L^{1}\left(-(a)^{2}, 0 ; L^{2 p}(B(a))\right)}\right] \cdot\left\|v_{1}\right\|_{L^{\infty}\left(-(a)^{2}, 0 ; W^{n, p}(B(a))\right)}^{p-\frac{1}{2}}
\end{aligned}
$$

Note that such constants are independent of any $v_{1}, v_{2}$ and $P$. 
Proof. See Appendix A.

The following non-local Sobolev-type lemma will be useful when we handle fractional derivatives by Maximal functions. We will see in Section 4.4 that the power $\left(1+\frac{3}{p}\right)$ of $M$ on the right-hand side of the following estimate is important to obtain the estimate (90).

Lemma 4.4. Let $M_{0}>0$ and $1 \leqslant p<\infty$. Then there exist $C=C\left(M_{0}, p\right)$ with the following property:

For any $M \geqslant M_{0}$ and for any $f \in C^{1}\left(\mathbb{R}^{3}\right)$ such that $\int_{\mathbb{R}^{3}} \phi(x) f(x) d x=0$, we have

$$
\|f\|_{L^{p}(B(M))} \leqslant C M^{1+\frac{3}{p}} \cdot\left(\left\|\mathcal{M}\left(|\nabla f|^{p}\right)\right\|_{L^{1}(B(1))}^{1 / p}+\|\nabla f\|_{L^{1}(B(2))}\right) .
$$

Proof. See Appendix A.

With the above lemmas, we are ready to prove Proposition 2.2.

Proof of Proposition 2.2. We divide this proof into three stages.

Stage 1 in Section 4.2: First, we will obtain an $L_{t}^{\infty} L_{x}^{2}$-local bound for $u$ by using the mean zero property of $u$ and $w$. Then, an $L^{\infty}$-local bound of $u$ follows thanks to the first local study Proposition 2.1.

Stage 2 in Section 4.3: We will get an $L^{\infty}$-local bound for $\nabla^{d} u$ for $d \geqslant 1$ by using an induction argument with a bootstrapping. This is not obvious especially when $r$ is large because $w$ depends on a non-local part of $u$ while our knowledge about the $L^{\infty}$-bound of $u$ from the stage 1 is only local.

Stage 3 in Section 4.4: We will achieve an $L^{\infty}$-local bound for $(-\Delta)^{\alpha / 2} \nabla^{d} u$ for $d \geqslant 1$ with $0<\alpha<2$ from the integral representation of the fractional Laplacian. The non-locality of this fractional operator will lead us to adopting more complicated conditions (see (23)).

\subsection{Stage 1: to obtain $L^{\infty}$-local bound for $u$}

First we suppose that $u$ satisfies all conditions of Proposition 2.2 without (23) (the condition (23) will be assumed only at the stage 3). Our goal is to find a sufficiently small $\bar{\eta}>0$, which should be independent of $r \in[0, \infty)$.

Assume $\bar{\eta} \leqslant 1$ and define $\bar{r}_{0}=\frac{1}{4}$ for this subsection. From (21), we get

$$
\|u\|_{L^{2}\left(-4,0 ; L^{6}(B(2))\right)} \leqslant C\|\nabla u\|_{L^{2}\left(-4,0 ; L^{2}(B(2))\right)} \leqslant C \cdot \bar{\eta} .
$$

From Corollary 3.2, if $r \geqslant \bar{r}_{0}$, then

$$
\|w\|_{L^{2}\left(-4,0 ; L^{\infty}(B(2))\right)} \leqslant C \cdot \bar{\eta} .
$$

On the other hand, if $0 \leqslant r<\bar{r}_{0}$, then

$$
\left\|w^{\prime}\right\|_{L^{2}\left(-4,0 ; L^{6}\left(B\left(\frac{7}{4}\right)\right)\right)} \leqslant C\|u\|_{L^{2}\left(-4,0 ; L^{6}(B(2))\right)} \leqslant C \bar{\eta}
$$

because $\phi_{r}$ is supported in $B(r) \subset B(\bar{r})$, and $w=u * \phi_{r}$ (see (8)). For $w^{\prime \prime}$,

$$
\begin{aligned}
\left\|w^{\prime \prime}\right\|_{L^{2}\left(-4,0 ; L^{\infty}(B(2))\right)} & \leqslant\|\| u * \phi_{r}\left\|_{L^{1}(B(1))}\right\|_{L^{2}((-4,0))} \leqslant\|\| u\left\|_{L^{1}(B(2))}\right\|_{L^{2}((-4,0))} \\
& \leqslant C\|u\|_{L^{2}\left(-4,0 ; L^{6}(B(2))\right)} \leqslant C \bar{\eta} .
\end{aligned}
$$

Thus $\|w\|_{L^{2}\left(-4,0 ; L^{6}\left(B\left(\frac{7}{4}\right)\right)\right)} \leqslant C \bar{\eta}$ if $r<\bar{r}_{0}$ from $w=w^{\prime}+w^{\prime \prime}$.

In sum, for any $0 \leqslant r<\infty$,

$$
\|w\|_{L^{2}\left(-4,0 ; L^{6}\left(B\left(\frac{7}{4}\right)\right)\right)} \leqslant C \bar{\eta} .
$$

Since Eq. (18) depends only on $\nabla P$, without loss of generality, we may assume $\int_{\mathbb{R}^{3}} \phi(x) P(t, x)=0$ for $t \in$ $(-4,0)$. Then with the mean zero property $(21)$ of $u$, we have

$$
\left\|\int_{\mathbb{R}^{3}} \phi(x) \nabla P(\cdot, x) d x\right\|_{L^{1}(-4,0)} \leqslant C \bar{\eta}^{\frac{1}{2}}
$$

after integrating in $x$. 
From Sobolev's inequality, we have

$$
\begin{aligned}
& \|\nabla P\|_{L^{1}\left(-4,0 ; L^{\frac{3}{2}}\left(B\left(\frac{7}{4}\right)\right)\right)} \leqslant C \bar{\eta}^{\frac{1}{2}} \text { and } \\
& \|P\|_{L^{1}\left(-4,0 ; L^{3}\left(B\left(\frac{7}{4}\right)\right)\right)} \leqslant C \bar{\eta}^{\frac{1}{2}} .
\end{aligned}
$$

Then we follow step 1 and step 2 of the proof of Proposition 10 in [41], we can obtain

$$
\|u\|_{L^{\infty}\left(-3,0 ; L^{\frac{3}{2}}\left(B\left(\frac{6}{4}\right)\right)\right)} \leqslant C \bar{\eta}^{\frac{1}{3}}
$$

and then

$$
\|u\|_{L^{\infty}\left(-2,0 ; L^{2}\left(B\left(\frac{5}{4}\right)\right)\right)} \leqslant C \bar{\eta}^{\frac{1}{4}}
$$

for $0 \leqslant r<\infty$. Details are omitted.

Finally, by taking $0<\bar{\eta}<1$ such that $C \bar{\eta}^{\frac{1}{4}} \leqslant \bar{\delta}$, we have all assumptions of Proposition 2.1. As a result, we have $|u(t, x)| \leqslant 1$ on $\left[-\frac{3}{2}, 0\right] \times B\left(\frac{1}{2}\right)$.

\subsection{Stage 2: to obtain $L^{\infty}$ local bound for $\nabla^{d} u$}

In this subsection, we cover only classical derivatives, i.e. the case $\alpha=0$. For any integer $d \geqslant 1$, our goal is to find $C_{d, 0}$ such that $\left|\left((-\Delta)^{\frac{0}{2}} \nabla^{d}\right) u(t, x)\right|=\left|\nabla^{d} u(t, x)\right| \leqslant C_{d, 0}$ on $\left(-\left(\frac{1}{3}\right)^{2}, 0\right) \times\left(B\left(\frac{1}{3}\right)\right)$.

We define strictly decreasing sequences of balls and parabolic cylinders from $\left(-\left(\frac{1}{2}\right)^{2}, 0\right) \times B\left(\frac{1}{2}\right)$ to $\left(-\left(\frac{1}{3}\right)^{2}, 0\right) \times$ $\left(B\left(\frac{1}{3}\right)\right)$ by

$$
\begin{aligned}
& \bar{B}_{n}=B\left(\frac{1}{3}+\frac{1}{6} \cdot 2^{-n}\right)=B\left(l_{n}\right), \\
& \bar{Q}_{n}=\left(-\left(\frac{1}{3}+\frac{1}{6} \cdot 2^{-n}\right)^{2}, 0\right) \times \bar{B}_{n}=\left(-\left(l_{n}\right)^{2}, 0\right) \times \bar{B}_{n}
\end{aligned}
$$

where $l_{n}=\frac{1}{3}+\frac{1}{6} \cdot 2^{-n}$. These notations will be used only in this subsection.

First, in order to cover the small $r$ case, we claim the following:

There exist two positive sequences $\left\{\bar{r}_{n}\right\}_{n=0}^{\infty}$ and $\left\{C_{n, \text { small }}\right\}_{n=0}^{\infty}$ such that for any integer $n \geqslant 0$ and for any $r \in\left[0, \bar{r}_{n}\right)$,

$$
\left\|\nabla^{n} u\right\|_{L^{\infty}\left(\bar{Q}_{11 n}\right)} \leqslant C_{n, \text { small }}
$$

Indeed, from the previous Section 4.2 (the stage 1), the estimate (82) holds for $n=0$ by taking $\bar{r}_{0}=1$ and $C_{0, \text { small }}=1$. We define $\bar{r}_{n}=$ distance between $B_{11 n}$ and $\left(B_{11 n-1}\right)^{C}$ for $n \geqslant 1$. Then $\left\{\bar{r}_{n}\right\}_{n=0}^{\infty}$ is decreasing to zero as $n$ goes to $\infty$. Moreover, we can control $w$ by $u$ as long as $0 \leqslant r<\bar{r}_{n}$ : for any $n \geqslant 1$,



$$
\begin{aligned}
& \left\|\nabla^{k} w\right\|_{L^{p_{1}\left(-\left(l_{m}\right)^{2}, 0 ; L^{p_{2}}\left(\bar{B}_{m}\right)\right)}} \leqslant\left\|\nabla^{k} u\right\|_{L^{p_{1}\left(-\left(l_{m-1}\right)^{2}, 0 ; L^{p_{2}}\left(\bar{B}_{m-1}\right)\right)}}
\end{aligned}
$$

for any integer $m$ such that $m \leqslant 11 \cdot n$, for any $k \geqslant 1$ and for any $p_{1} \in[1, \infty]$ and $p_{2} \in[1, \infty]$ (see (8)).

We will use an induction with a bootstrapping. First we fix $d \geqslant 1$ and suppose that (82) is true up to $n=(d-1)$. It implies for any $r \in\left[0, \bar{r}_{d-1}\right)$

$$
\|u\|_{L^{\infty}\left(-l_{s}^{2}, 0 ; W^{d-1, \infty}\left(\bar{B}_{s}\right)\right)} \leqslant C
$$

where $s=11(d-1)$. We want to show that (82) is also true for the case $n=d$.

From (83), $\|w\|_{L^{\infty}\left(-l_{s+1}^{2}, 0 ; W^{d-1, \infty}\left(\bar{B}_{s+1}\right)\right)} \leqslant C$ and, from Lemma 4.2 with $v_{2}=w$ and $v_{1}=u$, $\|u\|_{L^{16}\left(-l_{s+2}^{2}, 0 ; W^{d, 32}\left(\bar{B}_{s+2}\right)\right)} \leqslant C$. Then, we use (83) and Lemma 4.2 in turn:

$$
\begin{aligned}
& \rightarrow w \in L^{16}\left(-l_{s+3}^{2}, 0 ; W^{d, 32}\left(\bar{B}_{s+3}\right)\right) \rightarrow u \in L^{8}\left(-l_{s+4}^{2}, 0 ; W^{d+1,16}\left(\bar{B}_{s+4}\right)\right) \\
& \rightarrow w \in L^{8} W^{d+1,16} \rightarrow \cdots \rightarrow u \in L^{2} W^{d+3,4} .
\end{aligned}
$$


Then, from Sobolev's inequality,

$$
\rightarrow u \in L^{2} W^{d+2, \infty} \rightarrow w \in L^{2}\left(-l_{s+9}^{2}, 0 ; W^{d+2, \infty}\left(\bar{B}_{s+9}\right)\right) .
$$

This estimate gives us

$$
\Delta\left(\nabla^{d} u\right), \operatorname{div}\left(\nabla^{d}(w \otimes u)\right) \text { and } \nabla\left(\nabla^{d} P\right) \in L^{1}\left(-l_{s+10}^{2}, 0 ; L^{\infty}\left(\bar{B}_{s+10}\right)\right)
$$

where we used (79) for the pressure term. Thus

$$
\partial_{t}\left(\nabla^{d} u\right) \in L^{1}\left(-l_{s+10}^{2}, 0 ; L^{\infty}\left(\bar{B}_{s+10}\right)\right) .
$$

Finally, we obtain that, for any $r \in\left[0, \bar{r}_{d}\right)$

$$
\left\|\nabla^{d} u\right\|_{L^{\infty}\left(-l_{s+11}^{2}, 0 ; L^{\infty}\left(\bar{B}_{s+11}\right)\right)} \leqslant C
$$

where $C$ depends only on $d$. By this induction argument, we showed the above claim (82).

Now we introduce the second claim:

There exists a sequence $\left\{C_{n} \text {, large }\right\}_{n=0}^{\infty}$ such that for any integer $n \geqslant 0$ and for any $r \geqslant \bar{r}_{n}$,

$$
\left\|\nabla^{n} u\right\|_{L^{\infty}\left(\bar{Q}_{21 \cdot n}\right)} \leqslant C_{n, \text { large }}
$$

where $\bar{r}_{n}$ comes from the previous claim (82).

Before proving the above second claim (84), we need the following two observations (I), (II) from Lemmas 4.2 and 4.1:

(I). From Corollary 3.2 for any $n \geqslant 0$, if $r \geqslant \bar{r}_{n}$, then

$$
\|w\|_{L^{2}\left(-4,0 ; W^{n, \infty}(B(2))\right)} \leqslant C_{n} .
$$

We use (80) in Lemma 4.2 with $v_{1}=u$ and $v_{2}=w$. Then it becomes

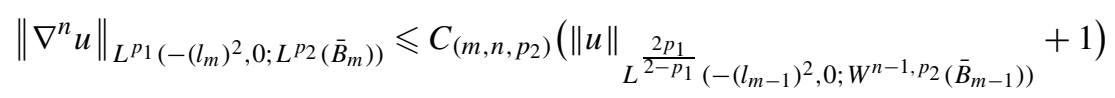

for $n \geqslant 1, m \geqslant 1,1<p_{1} \leqslant 2$ and $1<p_{2}<\infty$ (for the case $p_{1}=2$, we may interpret $\frac{2 p_{1}}{2-p_{1}}=\infty$ ).

(II). Moreover, (78) in Lemma 4.1 becomes

$$
\left\|\nabla^{n} P\right\|_{L^{1}\left(-\left(l_{m}\right)^{2}, 0 ; L^{p}\left(\bar{B}_{m}\right)\right)} \leqslant C_{(m, n, p)}\left(\|u\|_{L^{2}\left(-\left(l_{m-1}\right)^{2}, 0 ; W^{n-1, p}\left(\bar{B}_{m-1}\right)\right)}+1\right)
$$

for $n \geqslant 2$ and $1<p<\infty$.

Now we are ready to prove the second claim (84) by an induction with a bootstrapping. From the previous Section 4.2 (the stage 1), (84) holds for $n=0$ with $C_{0, \text { large }}=1$. Fix $d \geqslant 1$ and suppose that we have (84) up to $n=(d-1)$. It implies for any $r \geqslant \bar{r}_{d-1}$

$$
\|u\|_{L^{\infty}\left(-l_{s}^{2}, 0 ; W^{d-1, \infty}\left(\bar{B}_{s}\right)\right)} \leqslant C_{d-1, \text { large }}
$$

where $s=21(d-1)$. We want to show (84) for $n=d$.

By using (85) with $n=d, p_{1}=2$ and $p_{2}=11$, we have

$$
\|u\|_{L^{2}\left(-l_{s+1}^{2}, 0 ; W^{d, 11}\left(\bar{B}_{s+1}\right)\right)} \leqslant C
$$

and, from (86) with $n=d+1, m=0$ and $p=11$, we get

$$
\left\|\nabla^{d+1} P\right\|_{L^{1}\left(-l_{s+2}^{2}, 0 ; L^{11}\left(\bar{B}_{s+2}\right)\right)} \leqslant C .
$$

Combining the above two results with Lemma 4.3 for $v_{1}=u$ and $v_{2}=w$, we can increase the integrability in space of $u$ by 0.5 up to 6 :

$$
\begin{aligned}
& \|u\|_{L^{\infty}\left(-l_{s+3}^{2}, 0 ; W^{d, 1}\left(\bar{B}_{s+3}\right)\right)} \leqslant C, \\
& \|u\|_{L^{\infty}\left(-l_{s+4}^{2}, 0 ; W^{d, 1.5}\left(\bar{B}_{s+4}\right)\right)} \leqslant C, \\
& \vdots \\
& \|u\|_{L^{\infty}\left(-l_{s+13}^{2}, 0 ; W^{d, 6}\left(\bar{B}_{s+13))}\right.\right.} \leqslant C .
\end{aligned}
$$


By using (85) and (86) again, we have

$$
\begin{aligned}
& \|u\|_{L^{2}\left(-l_{s+14}^{2}, 0 ; W^{d+1,6}\left(\bar{B}_{s+14}\right)\right)} \leqslant C \quad \text { and } \\
& \left\|\nabla^{d+2} P\right\|_{L^{1}\left(-l_{s+15}^{2}, 0 ; L^{6}\left(\bar{B}_{s+15}\right)\right)} \leqslant C .
\end{aligned}
$$

Combining the above two results with Lemma 4.3 again, we have

$$
\begin{aligned}
& \|u\|_{L^{\infty}\left(-l_{s+16}^{2}, 0 ; W^{d+1,1}\left(\bar{B}_{s+16)}\right)\right.} \leqslant C, \\
& \quad \vdots \\
& \|u\|_{L^{\infty}\left(-l_{s+21}^{2}, 0 ; W^{d+1,3.5}\left(\bar{B}_{s+21}\right)\right)} \leqslant C .
\end{aligned}
$$

Finally, from Sobolev's inequality,

$$
\left\|\nabla^{d} u\right\|_{L^{\infty}\left(-l_{s+21}^{2}, 0 ; L^{\infty}\left(\bar{B}_{s+21}\right)\right)} \leqslant C
$$

where $C$ depends only on $d$ as long as $r \geqslant \bar{r}_{d}$. From this induction, we proved the second claim (84).

Define for any $n \geqslant 0, C_{n, 0}=\max \left(C_{n, \text { small }}, C_{n, \text { large }}\right)$ where $C_{n, \text { small }}$ and $C_{n, \text { large }}$ come from (82) and (84) respectively. Then we have:

$$
\left\|\nabla^{n} u\right\|_{L^{\infty}\left(Q\left(\frac{1}{3}\right)\right)} \leqslant C_{n, 0}
$$

for any $n \geqslant 0$ and for any $0 \leqslant r<\infty$ due to $Q\left(\frac{1}{3}\right) \subset \bar{Q}_{n}$. It ends this stage 2 .

\subsection{Stage 3: to obtain $L^{\infty}$ local bound for $(-\Delta)^{\alpha / 2} \nabla^{d} u$}

From now on, we assume further that $(u, P)$ satisfies (23) as well as all the other conditions of Proposition 2.2. In the following proof, we will not divide the proof into a small $r$ part and a large $r$ part.

Fix an integer $d \geqslant 1$ and a real $\alpha$ with $0<\alpha<2$. i.e. any constant which will appear may depend on $d$ and $\alpha$. However, it will be clear that all constants are independent of any $r \in[0, \infty)$ and of any solution $(u, P)$.

First, we claim:

There exists a constant $C=C(d, \alpha)$ such that

$$
\left|(-\Delta)^{\frac{\alpha}{2}} \nabla^{d} u(t, x)\right| \leqslant C(d, \alpha)+\left|\int_{|y| \geqslant(1 / 6)} \frac{\nabla^{d} u(t, x-y)}{|y|^{3+\alpha}} d y\right|
$$

for $|x| \leqslant(1 / 6)$ and for $-(1 / 3)^{2} \leqslant t \leqslant 0$.

To prove (88), we first recall the Taylor expansion of any $C^{2}$ function $f$ at $x: f(y)-f(x)=(\nabla f)(x) \cdot(y-x)+$ $R(x, y)$, and we have an error estimate $|R| \leqslant C|x-y|^{2} \cdot\left\|\nabla^{2} f\right\|_{L^{\infty}(B(x ;|x-y|))}$. Note that if we integrate the first order term $(\nabla f)(x) \cdot(y-x)$ in $y$ on any sphere with the center $x$, the integral vanishes thanks to the symmetry. As a result, if we take $(t, x)$ such that $|x| \leqslant(1 / 6)$ and $-(1 / 3)^{2} \leqslant t \leqslant 0$, then we have

$$
\begin{aligned}
\left|(-\Delta)^{\frac{\alpha}{2}} \nabla^{d} u(t, x)\right|= & \left|P . V \cdot \int_{\mathbb{R}^{3}} \frac{\nabla^{d} u(t, x)-\nabla^{d} u(t, y)}{|x-y|^{3+\alpha}} d y\right| \\
\leqslant & \sup _{z \in B((1 / 3))}\left(\left|\nabla^{d+2} u(t, z)\right|\right) \cdot \int_{|x-y|<(1 / 6)} \frac{1}{|x-y|^{3+\alpha-2}} d y \\
& +\sup _{z \in B((1 / 3))}\left(\left|\nabla^{d} u(t, z)\right|\right) \cdot \int_{|x-y| \geqslant(1 / 6)} \frac{1}{|x-y|^{3+\alpha}} d y+\left|\int_{|x-y| \geqslant(1 / 6)} \frac{\nabla^{d} u(t, y)}{|x-y|^{3+\alpha}} d y\right| \\
\leqslant & C(d, \alpha)+\left|\int_{|y| \geqslant(1 / 6)} \frac{\nabla^{d} u(t, x-y)}{|y|^{3+\alpha}} d y\right|
\end{aligned}
$$


where we used the result (87) of the previous Section 4.3 (the stage 2) together with the Taylor expansion of $\nabla^{d} u(t, \cdot)$ at $x$ in order to reduce certain amount of singularity at the origin $x=y$. We proved the first claim (88).

Second, we claim:

There exists $C=C(d, \alpha)$ such that

$$
\left|\int_{|y| \geqslant(1 / 6)} \frac{\nabla^{d} u(t, x-y)}{|y|^{3+\alpha}} d y\right| \leqslant C(d, \alpha)+\sum_{j=k}^{\infty}\left(\frac{1}{2^{\alpha}}\right)^{j} \cdot\left|\left(\left(h^{\alpha}\right)_{2^{j}} * \nabla^{d} u\right)(t, x)\right|
$$

for $|x| \leqslant(1 / 6)$ and for $-(1 / 3)^{2} \leqslant t \leqslant 0$ where $k$ is the integer such that $2^{k} \leqslant(1 / 6)<2^{k+1}$ (i.e. from now on, we fix $k=-3)$. Recall that $h^{\alpha}$ is defined in (12).

To prove the above second claim (89): (recall (11) and (12))

$$
\begin{aligned}
\int_{|y| \geqslant(1 / 6)} \frac{\nabla^{d} u(t, x-y)}{|y|^{3+\alpha}} d y \mid= & \left|\int_{|y| \geqslant(1 / 6)} \sum_{j=k}^{\infty} \zeta\left(\frac{y}{2^{j}}\right) \frac{\nabla^{d} u(t, x-y)}{|y|^{3+\alpha}} d y\right| \\
= & \left|\int_{|y| \geqslant(1 / 6)} \sum_{j=k}^{\infty} \frac{1}{\left(2^{j}\right)^{\alpha}} \cdot\left(h^{\alpha}\right)_{2^{j}}(y) \nabla^{d} u(t, x-y) d y\right| \\
\leqslant & \sum_{j=k}^{k+1} \frac{1}{\left(2^{j}\right)^{\alpha}} \cdot\left|\int_{|y| \geqslant(1 / 6)}\left(h^{\alpha}\right)_{2^{j}}(y) \nabla^{d} u(t, x-y) d y\right| \\
& +\sum_{j=k+2}^{\infty} \frac{1}{\left(2^{j}\right)^{\alpha}} \cdot\left|\int_{|y| \geqslant(1 / 6)}\left(h^{\alpha}\right)_{2^{j}}(y) \nabla^{d} u(t, x-y) d y\right| \\
= & (I)+(I I) .
\end{aligned}
$$

For $(I)$, we have

$$
\begin{aligned}
(I) & \leqslant \sum_{j=k}^{k+1} \frac{1}{\left(2^{j}\right)^{\alpha}} \cdot\left(\left|\int_{\mathbb{R}^{3}}\left(h^{\alpha}\right)_{2^{j}}(y) \nabla^{d} u(t, x-y) d y\right|+\int_{|y| \leqslant(1 / 6)}\left|\left(h^{\alpha}\right)_{2^{j}}(y)\right| \cdot\left|\nabla^{d} u(t, x-y)\right| d y\right) \\
& \leqslant \sum_{j=k}^{k+1} \frac{1}{\left(2^{j}\right)^{\alpha}}\left(\left|\left(\left(h^{\alpha}\right)_{2^{j}} * \nabla^{d} u\right)(t, x)\right|+C \cdot \sup _{z \in B(1 / 3)}\left|\nabla^{d} u(t, z)\right|\right) \\
& =\sum_{j=k}^{k+1}\left(\frac{1}{2^{\alpha}}\right)^{j} \cdot\left|\left(\left(h^{\alpha}\right)_{2^{j}} * \nabla^{d} u\right)(t, x)\right|+C(d, \alpha) .
\end{aligned}
$$

For (II), by using $\operatorname{supp}\left(h_{2^{j}}^{\alpha}\right) \subset\left(B\left(2^{j-1}\right)\right)^{C} \subset(B(1 / 6))^{C}$ for any $j \geqslant k+2$,

$$
\begin{aligned}
(I I) & =\sum_{j=k+2}^{\infty} \frac{1}{\left(2^{j}\right)^{\alpha}} \cdot\left|\int_{\mathbb{R}^{3}}\left(h^{\alpha}\right)_{2^{j}}(y) \nabla^{d} u(t, x-y) d y\right| \\
& =\sum_{j=k+2}^{\infty}\left(\frac{1}{2^{\alpha}}\right)^{j} \cdot\left|\left(\left(h^{\alpha}\right)_{2^{j}} * \nabla^{d} u\right)(t, x)\right| .
\end{aligned}
$$

We showed the second claim (89).

Third, we claim:

There exists $C=C(d, \alpha)$ such that

$$
\left\|\left(h^{\alpha}\right)_{M} * \nabla^{d} u\right\|_{L^{\infty}\left(-(1 / 6)^{2}, 0 ; L^{1}(B(1 / 6))\right)} \leqslant C(d, \alpha) \cdot M^{1-d}
$$

for any $M \geqslant 2^{k}$ (recall $k=-3$ ). 
To prove the above third claim (90), we take the convolution with $\nabla^{d}\left[\left(h^{\alpha}\right)_{M}\right]$ to Eq. (18). Then we have

$$
\left(\nabla^{d}\left[\left(h^{\alpha}\right)_{M}\right] * u\right)_{t}+\left(\nabla^{d}\left[\left(h^{\alpha}\right)_{M}\right] *((w \cdot \nabla) u)\right)+\left(\nabla^{d}\left[\left(h^{\alpha}\right)_{M}\right] * \nabla P\right)-\left(\nabla^{d}\left[\left(h^{\alpha}\right)_{M}\right] * \Delta u\right)=0
$$

so that we get

$$
\left(\nabla^{d-1}\left[\left(h^{\alpha}\right)_{M}\right] * \nabla u\right)_{t}+\left(\nabla^{d}\left[\left(h^{\alpha}\right)_{M}\right] *((w \cdot \nabla) u)\right)+\left(\nabla^{d-1}\left[\left(h^{\alpha}\right)_{M}\right] * \nabla^{2} P\right)-\Delta\left(\nabla^{d-1}\left[\left(h^{\alpha}\right)_{M}\right] * \nabla u\right)=0 .
$$

Define a cut-off $\Phi(t, x)$ by

$$
\begin{aligned}
& 0 \leqslant \Phi(x) \leqslant 1, \quad \operatorname{supp}(\Phi) \subset(-4,0) \times B(2), \\
& \Phi(t, x)=1 \quad \text { for }(t, x) \in\left(-(1 / 6)^{2}, 0\right) \times B((1 / 6)) .
\end{aligned}
$$

We multiply $\Phi(t, x) \frac{\left(\nabla^{d-1}\left[\left(h^{\alpha}\right)_{M}\right] * \nabla u\right)(t, x)}{\left|\left(\nabla^{d-1}\left[\left(h^{\alpha}\right)_{M}\right] * \nabla u\right)(t, x)\right|}$, then integrate in $x$ :

$$
\begin{aligned}
& \frac{d}{d t} \int_{\mathbb{R}^{3}} \Phi(t, x)\left|\left(\nabla^{d-1}\left[\left(h^{\alpha}\right)_{M}\right] * \nabla u\right)(t, x)\right| d x \\
& \leqslant \int_{\mathbb{R}^{3}}\left(\left|\partial_{t} \Phi(t, x)\right|+|\Delta \Phi(t, x)|\right)\left|\left(\nabla^{d-1}\left[\left(h^{\alpha}\right)_{M}\right] * \nabla u\right)(t, x)\right| d x \\
& \quad+\int_{\mathbb{R}^{3}}|\Phi(t, x)|\left|\left(\nabla^{d-1}\left[\left(h^{\alpha}\right)_{M}\right] * \nabla^{2} P\right)\right| d x+\int_{\mathbb{R}^{3}}|\Phi(t, x)|\left|\nabla^{d}\left[\left(h^{\alpha}\right)_{M}\right] *((w \cdot \nabla) u)\right| d x .
\end{aligned}
$$

Then the integration on $[-4, t]$ for any $t \in[-(1 / 6), 0]$ gives us

$$
\begin{aligned}
\left\|\left(h^{\alpha}\right)_{M} * \nabla^{d} u\right\|_{L^{\infty}\left(-(1 / 6)^{2}, 0 ; L^{1}(B(1 / 6))\right)}= & \left\|\nabla^{d-1}\left[\left(h^{\alpha}\right)_{M}\right] * \nabla u\right\|_{L^{\infty}\left(-(1 / 6)^{2}, 0 ; L^{1}(B(1 / 6))\right)} \\
\leqslant & C\left(\left\|\nabla^{d-1}\left[\left(h^{\alpha}\right)_{M}\right] * \nabla u\right\|_{L^{1}\left(-4,0 ; L^{1}(B(2))\right)}\right. \\
& +\left\|\nabla^{d-1}\left[\left(h^{\alpha}\right)_{M}\right] * \nabla^{2} P\right\|_{L^{1}\left(-4,0 ; L^{1}(B(2))\right)} \\
& \left.+\left\|\nabla^{d}\left[\left(h^{\alpha}\right)_{M}\right] *((w \cdot \nabla) u)\right\|_{L^{1}\left(-4,0 ; L^{1}(B(2))\right)}\right) \\
= & (I)+(I I)+(I I I) .
\end{aligned}
$$

For $(I)$, we use simple observations $\nabla^{m}\left[(f)_{\delta}\right]=\delta^{-m} \cdot\left(\nabla^{m} f\right)_{\delta}$ and $\left|(f)_{\delta} * \nabla u\right|(x) \leqslant C_{f} \cdot \mathcal{M}(|\nabla u|)(x)$ for any $f \in C_{0}^{\infty}\left(\mathbb{R}^{3}\right)$. Thus we get

$$
\begin{aligned}
\left|\left(\nabla^{d-1}\left[\left(h^{\alpha}\right)_{M}\right] * \nabla u\right)(t, x)\right| & =M^{-(d-1)} \cdot\left|\left(\left(\nabla^{d-1} h^{\alpha}\right)_{M} * \nabla u\right)(t, x)\right| \\
& \leqslant C \cdot M^{-(d-1)} \cdot \mathcal{M}(|\nabla u|)(t, x)
\end{aligned}
$$

for any $0<M<\infty$. It implies, for any $0<M<\infty$,

$$
\begin{aligned}
(I) & =\left\|\left(\nabla^{d-1}\left[\left(h^{\alpha}\right)_{M}\right] * \nabla u\right)\right\|_{L^{1}\left(-4,0 ; L^{1}(B(2))\right)} \\
& \leqslant C \cdot M^{-(d-1)} \cdot\|\mathcal{M}(|\nabla u|)\|_{L^{1}\left(-4,0 ; L^{1}(B(2))\right)} \\
& \leqslant C \cdot M^{-(d-1)} \cdot\|\mathcal{M}(|\nabla u|)\|_{L^{2}\left(-4,0 ; L^{2}(B(2))\right)} \leqslant C \cdot M^{1-d} .
\end{aligned}
$$

For $(I I)$, we use our global information about the pressure in (23) thanks to the property of the Hardy space (10):

$$
\begin{aligned}
(I I) & =\left\|\nabla^{d-1}\left[\left(h^{\alpha}\right)_{M}\right] * \nabla^{2} P\right\|_{L^{1}\left(-4,0 ; L^{1}(B(2))\right)} \\
& =M^{-(d-1)} \cdot\left\|\left(\nabla^{d-1} h^{\alpha}\right)_{M} * \nabla^{2} P\right\|_{L^{1}\left(-4,0 ; L^{1}(B(2))\right)} \\
& \leqslant M^{-(d-1)} \cdot\left\|\sup _{\delta>0}\left(\left|\left(\nabla^{d-1} h^{\alpha}\right)_{\delta} * \nabla^{2} P\right|\right)\right\|_{L^{1}\left(-4,0 ; L^{1}(B(2))\right)} \\
& \leqslant C \cdot M^{1-d}
\end{aligned}
$$

for any $0<M<\infty$. 
For $(I I I)$, we use the following useful facts $(\mathbf{1}-\mathbf{5})$ :

1. From $\operatorname{supp}\left(\left(h^{\alpha}\right)_{M}\right) \subset B(2 M)$, we compute

$$
\begin{aligned}
& \left\|\nabla^{d}\left[\left(h^{\alpha}\right)_{M}\right] *((w \cdot \nabla) u)(t, \cdot)\right\|_{L^{1}(B(2))} \\
& \quad \leqslant \int_{B(2)} \int_{\mathbb{R}^{3}}\left|((w \cdot \nabla) u)(t, y) \cdot\left(\nabla^{d}\left[\left(h^{\alpha}\right)_{M}\right]\right)(x-y)\right| d y d x \\
& \quad \leqslant \int_{B(2 M+2)}|((w \cdot \nabla) u)(t, y)| \cdot\left[\int_{B(2)}\left|\left(\nabla^{d}\left[\left(h^{\alpha}\right)_{M}\right]\right)(x-y)\right| d x\right] d y \\
& \quad \leqslant C\left\|\nabla^{d}\left[\left(h^{\alpha}\right)_{M}\right]\right\|_{L^{\infty}\left(\mathbb{R}^{3}\right)} \cdot\|((w \cdot \nabla) u)(t, \cdot)\|_{L^{1}(B(2 M+2))} \\
& \leqslant C \cdot \frac{1}{M^{3+d}} \cdot\|((w \cdot \nabla) u)(t, \cdot)\|_{L^{1}(B(2 M+2))} \\
& \leqslant C \cdot \frac{1}{M^{3+d}} \cdot\|w(t, \cdot)\|_{L^{q^{\prime}(B(2 M+2))}} \cdot\|\nabla u(t, \cdot)\|_{L^{q}(B(2 M+2))}
\end{aligned}
$$

where $q=12 /(\alpha+6)$ and $1 / q+1 / q^{\prime}=1$. Note that $12 / 8<q<2$ due to $0<\alpha<2$.

2. For any $M \geqslant 2^{k}$, we have

$$
\begin{aligned}
\|w(t, \cdot)\|_{L^{q}(B(2 M+2))} & \leqslant C M^{1+\frac{3}{q}} \cdot\left(\left\|\mathcal{M}\left(|\nabla w|^{q}\right)(t, \cdot)\right\|_{L^{1}(B(1))}^{1 / q}+\|\nabla w(t, \cdot)\|_{L^{1}(B(2))}\right) \\
& \leqslant C M^{1+\frac{3}{q}} \cdot\left(\left\|\mathcal{M}\left(|\mathcal{M}(|\nabla u|)|^{q}\right)(t, \cdot)\right\|_{L^{1}(B(1))}^{1 / q}+\|\mathcal{M}(|\nabla u|)(t, \cdot)\|_{L^{1}(B(2))}\right),
\end{aligned}
$$

where, for the first inequality, we used Lemma 4.4 and, for the second one, we used the fact $|\nabla w(t, x)|=\mid(\nabla u *$ $\left.\phi_{r}\right)(t, x) \mid \leqslant C \cdot \mathcal{M}(|\nabla u|)(t, x)$. Note that $C$ is independent of $r \in[0, \infty)$ thanks to the definitions of the convolution and the Maximal function. So, for any $M \geqslant 2^{k}$, from (23), we get

$$
\begin{aligned}
\|w\|_{L^{2}\left(-4,0 ; L^{q}(B(2 M+2))\right)} & \leqslant C M^{1+\frac{3}{q}}\left(\|\| \mathcal{M}\left(|\mathcal{M}(|\nabla u|)|^{q}\right)\left\|_{L_{x}^{1}(B(1))}^{1 / q}\right\|_{L_{t}^{2}(-4,0)}+\|\| \mathcal{M}(|\nabla u|)\left\|_{L_{x}^{1}(B(2))}\right\|_{L_{t}^{2}(-4,0)}\right) \\
& \leqslant C M^{1+\frac{3}{q}}\left(\left\|\mathcal{M}\left(|\mathcal{M}(|\nabla u|)|^{q}\right)\right\|_{L^{2 / q}\left(-4,0 ; L^{1}(B(2))\right)}^{1 / q}+\|\mathcal{M}(|\nabla u|)\|_{L^{2}\left(-4,0 ; L^{1}(B(2))\right)}\right) \\
& \leqslant C M^{1+\frac{3}{q}}
\end{aligned}
$$

Before stating the third fact, we need the following two observations:

From the standard Sobolev-Poincaré inequality on balls (e.g. see Saloff-Coste [32]), we have, for any $0<M<\infty$ and for any $f$ whose derivatives are in $L_{l o c}^{q}\left(\mathbb{R}^{3}\right)$,

$$
\|f-\bar{f}\|_{L^{3 q /(3-q)(B(M))}} \leqslant C \cdot\|\nabla f\|_{L^{q}(B(M))}
$$

where $\bar{f}=\int_{B} f d x /|B|$ is the mean value on $B$. Note that $C$ is independent of $M$.

On the other hand, once we fix $M_{0}>0$, then there exists $C=C\left(M_{0}\right)$ with the following property:

For any $p$ with $1 \leqslant p<\infty$, for any $M \geqslant M_{0}$ and for any $f \in L_{l o c}^{p}\left(\mathbb{R}^{3}\right)$, we have

$$
\|f\|_{L^{p}(B(M))} \leqslant C M^{\frac{3}{p}} \cdot\left\|\mathcal{M}\left(|f|^{p}\right)\right\|_{L^{1}(B(2))}^{1 / p} .
$$

Indeed, to prove (93), it is enough to show that

$$
\|g\|_{L^{1}(B(M))} \leqslant C M^{3} \cdot\|\mathcal{M}(g)\|_{L^{1}(B(2))} .
$$

For any $z \in B(2)$, we get

$$
\begin{aligned}
\int_{B(M)}|g(x)| d x & =\frac{(M+2)^{3}}{(M+2)^{3}} \cdot \int_{B(M+2)}|g(z+x)| d x \\
& \leqslant(M+2)^{3} \mathcal{M}(g)(z) \leqslant C_{M_{0}} M^{3} \mathcal{M}(g)(z) .
\end{aligned}
$$

Then we take integral on $z \in B(2)$. 
Now we state the third fact.

3. $\|w(t, \cdot)\|_{L^{3 q /(3-q)(B(2 M+2))}}$

$$
\begin{aligned}
\leqslant & C \cdot\|\nabla w(t, \cdot)\|_{L^{q}(B(2 M+2))}+\|\bar{w}(t, \cdot)\|_{L^{3 q /(3-q)}(B(2 M+2))} \\
\leqslant & C \cdot M^{3 / q} \cdot\left\|\mathcal{M}\left(|\nabla w|^{q}\right)(t, \cdot)\right\|_{L^{1}(B(2))}^{1 / q}+C M^{-3}\|w(t, \cdot)\|_{L^{1}(B(2 M+2))} \cdot C M^{3 \cdot \frac{3-q}{3 q}} \\
\leqslant & C \cdot M^{3 / q} \cdot\left\|\mathcal{M}\left(|\mathcal{M}(|\nabla u|)|^{q}\right)(t, \cdot)\right\|_{L^{1}(B(2))}^{1 / q}+C M^{\frac{3}{q}-4}\|w(t, \cdot)\|_{L^{1}(B(2 M+2))} \\
\leqslant & C \cdot M^{3 / q} \cdot\left\|\mathcal{M}\left(|\mathcal{M}(|\nabla u|)|^{q}\right)(t, \cdot)\right\|_{L^{1}(B(2))}^{1 / q} \\
& +C M^{\frac{3}{q}-4} C M^{1+\frac{3}{1}} \cdot\left(\left\|\mathcal{M}\left(|\nabla w|^{1}\right)(t, \cdot)\right\|_{L^{1}(B(1))}^{1 / 1}+\|\nabla w(t, \cdot)\|_{L^{1}(B(2))}\right) \\
\leqslant & C \cdot M^{3 / q} \cdot\left\|\mathcal{M}\left(|\mathcal{M}(|\nabla u|)|^{q}\right)(t, \cdot)\right\|_{L^{1}(B(2))}^{1 / q} \\
& +C M^{\frac{3}{q}}\left(\|\mathcal{M}(|\mathcal{M}(|\nabla u|)|)(t, \cdot)\|_{L^{1}(B(1))}+\|\mathcal{M}(|\nabla u|)(t, \cdot)\|_{L^{1}(B(2))}\right)
\end{aligned}
$$

where we used (92) for the first inequality while we used (93) and definition of mean value for the second one. For fourth and fifth ones, $|\nabla w(t, x)| \leqslant C|\mathcal{M}(|\nabla u|)(t, x)|$ and Lemma 4.4 were used. So, by taking $L^{2}$-norm on time $[-4,0]$ with (23), we get

$$
\|w\|_{L^{2}\left(-4,0 ; L^{\frac{3 q}{3-q}}(B(2 M+2))\right)} \leqslant C M^{\frac{3}{q}}
$$

for any $M \geqslant 2^{k}$.

4. $\|w(t, \cdot)\|_{L^{q^{\prime}(B(2 M+2))}} \leqslant\|w(t, \cdot)\|_{L^{q}(B(2 M+2))}^{\theta} \cdot\|w(t, \cdot)\|_{L^{3 q /(3-q)}(B(2 M+2))}^{1-\theta}$

where $q^{\prime}=q /(q-1)$ and $\theta=(4 q-6) / q$.

Note that due to $12 / 8<q<2$, we have $0<\theta<1$. So, for any $M \geqslant 2^{k}$, we get

$$
\begin{aligned}
& \|w\|_{L^{2}\left(-4,0 ; L^{q^{\prime}}(B(2 M+2))\right)} \leqslant\|w\|_{L^{2}\left(-4,0 ; L^{q}(B(2 M+2))\right)}^{\theta} \cdot\|w\|_{L^{2}\left(-4,0 ; L^{3 q /(3-q)}(B(2 M+2))\right)}^{1-\theta} \\
& \leqslant C \cdot\left(M^{1+(3 / q)}\right)^{\theta}\left(M^{3 / q}\right)^{1-\theta}=C \cdot M^{4-\frac{3}{q}} .
\end{aligned}
$$

5. From (93), for any $M \geqslant 2^{k}$, we get

$$
\|\nabla u(t, \cdot)\|_{L^{q}(B(2 M+2))} \leqslant C \cdot M^{3 / q} \cdot\left\|\mathcal{M}\left(|\nabla u|^{q}\right)(t, \cdot)\right\|_{L^{1}(B(2))}^{1 / q} .
$$

So, for any $M \geqslant 2^{k}$, from (23), we obtain

$$
\begin{aligned}
\|\nabla u\|_{L^{2}\left(-4,0 ; L^{q}(B(2 M+2))\right)} & \leqslant C \cdot M^{3 / q} \cdot\|\| \mathcal{M}\left(|\nabla u|^{q}\right)\left\|_{L_{x}^{1}(B(2))}^{1 / q}\right\|_{L_{t}^{2}(-4,0)} \\
& \leqslant C \cdot M^{3 / q} \cdot\left\|\mathcal{M}\left(|\nabla u|^{q}\right)\right\|_{L^{2 / q}\left(-4,0 ; L^{1}(B(2))\right)}^{1 / q} \leqslant C \cdot M^{3 / q} .
\end{aligned}
$$

Using the above results (1-5) all together, we have, for any $M \geqslant 2^{k}$,

$$
\begin{aligned}
(I I I) & \leqslant C \cdot \frac{1}{M^{3+d}} \cdot\|w\|_{L^{2}\left(-4,0 ; L^{q^{\prime}}(B(2 M+2))\right)}\|\nabla u\|_{L^{2}\left(-4,0 ; L^{q}(B(2 M+2))\right)} \\
& \leqslant C \cdot \frac{1}{M^{3+d}} \cdot M^{4-(3 / q)} \cdot M^{3 / q}=C \cdot M^{1-d} .
\end{aligned}
$$

It proves the above third claim (90). 
Finally we combine three claims (88), (89) and (90):

$$
\begin{aligned}
& \left\|(-\Delta)^{\frac{\alpha}{2}} \nabla^{d} u\right\|_{L^{\infty}\left(-(1 / 6)^{2}, 0 ; L^{1}(B((1 / 6)))\right)} \\
& \leqslant\left\|C\left(1+\left|\int_{|y| \geqslant(1 / 6)} \frac{\nabla^{d} u\left(\cdot{ }^{\prime}, \cdot x-y\right)}{|y|^{3+\alpha}} d y\right|\right)\right\|_{L^{\infty}\left(-(1 / 6)^{2}, 0 ; L^{1}(B((1 / 6)))\right)} \\
& \leqslant C+C \sum_{j=k}^{\infty}\left(\frac{1}{2^{\alpha}}\right)^{j} \cdot\left\|\left|\left(\left(h^{\alpha}\right)_{2^{j}} * \nabla^{d} u\right)\left(\cdot{ }_{t}, \cdot x\right)\right|\right\|_{L^{\infty}\left(-(1 / 6)^{2}, 0 ; L^{1}(B((1 / 6)))\right)} \\
& \leqslant C+C \sum_{j=k}^{\infty}\left(\frac{1}{2^{\alpha}}\right)^{j} \cdot\left(2^{j}\right)^{1-d} \leqslant C+C \sum_{j=k}^{\infty}\left(\frac{1}{2^{d+\alpha-1}}\right)^{j} \leqslant C
\end{aligned}
$$

thanks to $d+\alpha-1>0$ from $d \geqslant 1$ and $\alpha>0$.

By the exact same way, we can also prove that

$$
\left\|(-\Delta)^{\frac{\alpha}{2}} \nabla^{m} u\right\|_{L^{\infty}\left(-(1 / 6)^{2}, 0 ; L^{1}(B((1 / 6)))\right)} \leqslant C
$$

for $m=d+1, \ldots, d+4$. By repeated uses of Sobolev's inequality, we get

$$
\left\|(-\Delta)^{\frac{\alpha}{2}} \nabla^{d} u\right\|_{L^{\infty}\left(-(1 / 6)^{2}, 0 ; L^{\infty}(B((1 / 6)))\right)} \leqslant C(d, \alpha) .
$$

It finishes the proof of Proposition 2.2.

\section{Proof of the main Theorem 1.1}

We begin this section by presenting one lemma about pivot quantities. After that, Section 5.2 will cover the part (II) for $\alpha=0$ while Section 5.3 does the part (II) for $0<\alpha<2$. Finally the part (I) for $0 \leqslant \alpha<2$ will be proved in Section 5.4.

\section{1. $L^{1}$ Pivot quantities}

The following lemma says that $L^{1}$ space-time norm of our pivot quantities can be controlled by $L^{2}$ space norm of the initial data. These quantities have the best scaling as $|\nabla u|^{2}$ and $\left|\nabla^{2} P\right|$ have among all other a priori quantities from $L^{2}$ initial data (also see (4)).

Lemma 5.1. There exist constant $C>0$ and $C_{d, \alpha}$ for integer $d \geqslant 1$ and real $\alpha \in(0,2)$ with the following property:

If $(u, P)$ is a solution of (Problem I-n) for some $1 \leqslant n \leqslant \infty$, then we have

$$
\int_{0}^{\infty} \int_{\mathbb{R}^{3}}\left(|\nabla u(t, x)|^{2}+\left|\nabla^{2} P(t, x)\right|+|\mathcal{M}(|\nabla u|)(t, x)|^{2}\right) d x d t \leqslant C\left\|u_{0}\right\|_{L^{2}\left(\mathbb{R}^{3}\right)}^{2}
$$

and

$$
\begin{gathered}
\int_{0}^{\infty} \int_{\mathbb{R}^{3}}\left(|\mathcal{M}(\mathcal{M}(|\nabla u|))|^{2}+\left|\mathcal{M}\left(|\nabla u|^{q}\right)\right|^{2 / q}+\left|\mathcal{M}\left(|\mathcal{M}(|\nabla u|)|^{q}\right)\right|^{2 / q}\right. \\
\left.+\sum_{m=d}^{d+4} \sup _{\delta>0}\left(\left|\left(\nabla^{m-1} h^{\alpha}\right)_{\delta} * \nabla^{2} P\right|\right)\right) d x d t \leqslant C_{d, \alpha}\left\|u_{0}\right\|_{L^{2}\left(\mathbb{R}^{3}\right)}^{2}
\end{gathered}
$$

for any integer $d \geqslant 1$ and any real $\alpha \in(0,2)$ where $q=q(\alpha)$ is defined by $12 /(\alpha+6)$.

Remark 5.1. The definitions of $h^{\alpha}$ and $\left(\nabla^{m-1} h^{\alpha}\right)_{\delta}$ can be found in (12). 
Remark 5.2. In the proof, we will see that every quantity in the left-hand sides of the above two estimates can be controlled by dissipation of energy $\|\nabla u\|_{L^{2}\left((0, \infty) \times \mathbb{R}^{3}\right)}^{2}$ only. It explains the latter part of Remark 1.2.

Proof of Lemma 5.1. From (17), we have

$$
\|\nabla u\|_{L^{2}\left(0, \infty ; L^{2}\left(\mathbb{R}^{3}\right)\right)}^{2} \leqslant\left\|u_{0} * \phi_{\frac{1}{n}}\right\|_{L^{2}\left(\mathbb{R}^{3}\right)}^{2} \leqslant\left\|u_{0}\right\|_{L^{2}\left(\mathbb{R}^{3}\right)}^{2} .
$$

For the pressure term, we use boundedness of the Riesz transform on Hardy space $\mathcal{H}$ and the compensated compactness result in Coifman, Lions, Meyer and Semmes [11]:

$$
\begin{aligned}
\left\|\nabla^{2} P\right\|_{L^{1}\left(0, \infty ; L^{1}\left(\mathbb{R}^{3}\right)\right)} & \leqslant\left\|\nabla^{2} P\right\|_{L^{1}\left(0, \infty ; \mathcal{H}\left(\mathbb{R}^{3}\right)\right)} \leqslant C\|\Delta P\|_{L^{1}\left(0, \infty ; \mathcal{H}\left(\mathbb{R}^{3}\right)\right)} \\
& =\left\|\operatorname{div} \operatorname{div}\left(\left(u * \phi_{1 / n}\right) \otimes u\right)\right\|_{L^{1}\left(0, \infty ; \mathcal{H}\left(\mathbb{R}^{3}\right)\right)} \\
& \leqslant C \cdot\left\|\nabla\left(u * \phi_{1 / n}\right)\right\|_{L^{2}\left(0, \infty ; L^{2}\left(\mathbb{R}^{3}\right)\right)}\|\nabla u\|_{L^{2}\left(0, \infty ; L^{2}\left(\mathbb{R}^{3}\right)\right)} \\
& \leqslant C \cdot\|\nabla u\|_{L^{2}\left(0, \infty ; L^{2}\left(\mathbb{R}^{3}\right)\right)}^{2} \leqslant C\left\|u_{0}\right\|_{L^{2}\left(\mathbb{R}^{3}\right)}^{2} .
\end{aligned}
$$

For Maximal functions, we have

$$
\begin{aligned}
\|\mathcal{M}(\mathcal{M}(|\nabla u|))\|_{L^{2}\left(0, \infty ; L^{2}\left(\mathbb{R}^{3}\right)\right)}^{2} & \leqslant C \cdot\|\mathcal{M}(|\nabla u|)\|_{L^{2}\left(0, \infty ; L^{2}\left(\mathbb{R}^{3}\right)\right)}^{2} \\
& \leqslant C \cdot\|\nabla u\|_{L^{2}\left(0, \infty ; L^{2}\left(\mathbb{R}^{3}\right)\right)}^{2} \\
& \leqslant C \cdot\left\|u_{0}\right\|_{L^{2}\left(\mathbb{R}^{3}\right)}^{2} .
\end{aligned}
$$

Let $d \geqslant 1$ and $0<\alpha<2$ and take $q=12 /(\alpha+6)$. Due to $1<(2 / q)<(4 / 3)$, we get

$$
\begin{aligned}
\left\|\mathcal{M}\left(|\nabla u|^{q}\right)\right\|_{L^{2 / q}\left(0, \infty ; L^{2 / q}\left(\mathbb{R}^{3}\right)\right)}^{2 / q} & \leqslant C \cdot\left\||\nabla u|^{q}\right\|_{L^{2 / q}\left(0, \infty ; L^{2 / q}\left(\mathbb{R}^{3}\right)\right)}^{2 / q} \\
& =C \cdot\|\nabla u\|_{L^{2}\left(0, \infty ; L^{2}\left(\mathbb{R}^{3}\right)\right)}^{2} \\
& \leqslant C \cdot\left\|u_{0}\right\|_{L^{2}\left(\mathbb{R}^{3}\right)}^{2}
\end{aligned}
$$

and

$$
\begin{aligned}
\left\|\mathcal{M}\left(|\mathcal{M}(|\nabla u|)|^{q}\right)\right\|_{L^{2 / q}\left(0, \infty ; L^{2 / q}\left(\mathbb{R}^{3}\right)\right)}^{2 / q} & \leqslant C \cdot\left\||\mathcal{M}(|\nabla u|)|^{q}\right\|_{L^{2 / q}\left(0, \infty ; L^{2 / q}\left(\mathbb{R}^{3}\right)\right)}^{2 / q} \\
& \leqslant C \cdot\|\mathcal{M}(|\nabla u|)\|_{L^{2}\left(0, \infty ; L^{2}\left(\mathbb{R}^{3}\right)\right)}^{2} \\
& \leqslant C \cdot\|\nabla u\|_{L^{2}\left(0, \infty ; L^{2}\left(\mathbb{R}^{3}\right)\right)}^{2} \\
& \leqslant C \cdot\left\|u_{0}\right\|_{L^{2}\left(\mathbb{R}^{3}\right)}^{2}
\end{aligned}
$$

where $C$ depends only on $\alpha$.

Thanks to the property of Hardy space (10) with (94), we have

$$
\begin{aligned}
\sum_{m=d}^{d+4}\left\|\sup _{\delta>0}\left(\left|\left(\nabla^{m-1} h^{\alpha}\right)_{\delta} * \nabla^{2} P\right|\right)\right\|_{L^{1}\left(0, \infty ; L^{1}\left(\mathbb{R}^{3}\right)\right)} & \leqslant \sum_{m=d}^{d+4} C\left\|\nabla^{2} P\right\|_{L^{1}\left(0, \infty ; \mathcal{H}\left(\mathbb{R}^{3}\right)\right)} \\
& \leqslant C\left\|u_{0}\right\|_{L^{2}\left(\mathbb{R}^{3}\right)}^{2}
\end{aligned}
$$

where the above $C$ depends only on $d$ and $\alpha$.

We are ready to prove the main Theorem 1.1.

Remark 5.3. In the following Sections 5.2 and 5.3, we consider solutions of (Problem I- $n$ ) for positive integers $n$. However it will be clear that every computation in these subsections can also be verified for the case $n=\infty$ once we assume that the smooth solution $u$ of the Navier-Stokes exists. The $n=\infty$ case (the original Navier-Stokes) will be covered in Section 5.4.

We focus on the $\alpha=0$ case of the part (II) first. 


\subsection{Proof of Theorem 1.1, part (II) for the $\alpha=0$ case}

Proof of Theorem 1.1, part (II) for the $\boldsymbol{\alpha}=\mathbf{0}$ case. Let any initial data $u_{0}$ of (2) be given. From Leray's construction, there exists the $C^{\infty}$ solution sequence $\left\{u_{n}\right\}_{n=1}^{\infty}$ of (Problem I- $n$ ) on $(0, \infty)$ with corresponding pressures $\left\{P_{n}\right\}_{n=1}^{\infty}$. From now on, our goal is to make an estimate for $\nabla^{d} u_{n}$ which is uniform in $n$.

For each $n, \epsilon>0, t>0$ and $x \in \mathbb{R}^{3}$, define a new flow $X_{n, \epsilon}(\cdot, t, x)$ by solving

$$
\begin{aligned}
& \frac{\partial X_{n, \epsilon}}{\partial s}(s, t, x)=u_{n} * \phi_{\frac{1}{n}} * \phi_{\epsilon}\left(s, X_{n, \epsilon}(s, t, x)\right) \text { for } s \in[0, t], \\
& X_{n, \epsilon}(t, t, x)=x .
\end{aligned}
$$

For convenience, we define $F_{n}(t, x)$ and $g_{n}(t)$.

$$
F_{n}(t, x)=\left(\left|\nabla u_{n}\right|^{2}+\left|\nabla^{2} P_{n}\right|+\left|\mathcal{M}\left(\nabla u_{n}\right)\right|^{2}\right)(t, x), \quad g_{n}(t)=\int_{\mathbb{R}^{3}} F_{n}(t, x) d x .
$$

We define, for $n, t>0$ and $\epsilon$ such that $0<4 \epsilon^{2} \leqslant t$,

$$
\Omega_{n, \epsilon, t}=\left\{x \in \mathbb{R}^{3} \mid \frac{1}{\epsilon} \int_{t-4 \epsilon^{2}}^{t} \int_{B(2 \epsilon)} F_{n}\left(s, X_{n, \epsilon}(s, t, x)+y\right) d y d s \leqslant \bar{\eta}\right\}
$$

where $\bar{\eta}$ comes from Proposition 2.2. We estimate the size of $\left(\Omega_{n, \epsilon, t}\right)^{C}$ :

$$
\begin{aligned}
\left|\left(\Omega_{n, \epsilon, t}\right)^{C}\right| & =\left|\left\{x \in \mathbb{R}^{3} \mid \frac{1}{\epsilon} \int_{t-4 \epsilon^{2}}^{t} \int_{B(2 \epsilon)} F_{n}\left(s, X_{n, \epsilon}(s, t, x)+y\right) d y d s>\bar{\eta}\right\}\right| \\
& \leqslant \frac{1}{\bar{\eta}} \int_{\mathbb{R}^{3}}\left(\frac{1}{\epsilon} \int_{t-4 \epsilon^{2}}^{t} \int_{B(2 \epsilon)} F_{n}\left(s, X_{n, \epsilon}(s, t, x)+y\right) d y d s\right) d x \\
& =\frac{1}{\bar{\eta} \epsilon}\left(\int_{B(2 \epsilon)} \int_{-4 \epsilon^{2}}^{0} \int_{\mathbb{R}^{3}} F_{n}\left(t+s, X_{n, \epsilon}(t+s, t, x)+y\right) d x d s d y\right) \\
& =\frac{1}{\bar{\eta} \epsilon}\left(\int_{B(2 \epsilon)}^{0} \int_{-4 \epsilon^{2}} \int_{\mathbb{R}^{3}} F_{n}(t+s, z+y) d z d s d y\right) \\
& \leqslant \frac{1}{\bar{\eta} \epsilon}\left(\int_{B(2 \epsilon)} 1 d y\right)\left(\int_{-4 \epsilon^{2}}^{0} \int_{\mathbb{R}^{3}} F_{n}(t+s, \bar{z}) d \bar{z} d s\right) \\
& \leqslant \frac{C \epsilon^{2}}{\bar{\eta}}\left(\int_{-4 \epsilon^{2}}^{0} \int_{\mathbb{R}^{3}} F_{n}(t+s, \bar{z}) d \bar{z} d s\right) \\
& \leqslant C \frac{\epsilon^{4}}{\bar{\eta}}\left(\frac{1}{4 \epsilon^{2}} \int_{-4 \epsilon^{2}}^{0} g_{n}(t+s) d s\right) \leqslant \epsilon^{4} \mathcal{M}^{(t)}\left(\frac{C}{\bar{\eta}} g_{n} \cdot \mathbf{1}_{(0, \infty)}\right)(t)=\epsilon^{4} \tilde{g}_{n}(t)
\end{aligned}
$$

where $\tilde{g}_{n}=\mathcal{M}^{(t)}\left(\frac{C}{\bar{\eta}} g_{n} \cdot \mathbf{1}_{(0, \infty)}\right)$ and $\mathcal{M}^{(t)}$ is the Maximal function in $\mathbb{R}^{1}$. For the third inequality, we used the fact that $X_{n, \epsilon}(\cdot, t, x)$ is incompressible. From the fact that the Maximal operator is bounded from $L^{1}$ to $L^{1, \infty}$ together with Lemma 5.1, we get $\left\|\tilde{g}_{n}(\cdot)\right\|_{L^{1, \infty}(0, \infty)} \leqslant \frac{C}{\bar{\eta}}\left\|g_{n}(\cdot)\right\|_{L^{1}(0, \infty)} \leqslant \frac{C}{\bar{\eta}}\left\|u_{0}\right\|_{L^{2}\left(\mathbb{R}^{3}\right)}^{2}$. 
Now we fix $n, t, \epsilon$ and $x$ with $n \geqslant 1,0<t<\infty, 0<4 \epsilon^{2} \leqslant t$ and $x \in \Omega_{n, \epsilon, t}$. We define $v, Q$ on $(-4, \infty) \times \mathbb{R}^{3}$ by using the Galilean invariance:

$$
\begin{aligned}
& v(s, y)=\epsilon u_{n}\left(t+\epsilon^{2} s, X_{n, \epsilon}\left(t+\epsilon^{2} s, t, x\right)+\epsilon y\right)-\epsilon\left(u_{n} * \phi_{\epsilon}\right)\left(t+\epsilon^{2} s, X_{n, \epsilon}\left(t+\epsilon^{2} s, t, x\right)\right), \\
& Q(s, y)=\epsilon^{2} P_{n}\left(t+\epsilon^{2} s, X_{n, \epsilon}\left(t+\epsilon^{2} s, t, x\right)+\epsilon y\right)+\epsilon y \partial_{s}\left[\left(u_{n} * \phi_{\epsilon}\right)\left(t+\epsilon^{2} s, X_{n, \epsilon}\left(t+\epsilon^{2} s, t, x\right)\right)\right] .
\end{aligned}
$$

Remark 5.4. This specially designed $\epsilon$-scaling will give the mean zero property to both the velocity and the convective velocity of the resulting equation (97).

Let us denote $\square$ and $\diamond$ by $\square=\left(t+\epsilon^{2} s, X_{n, \epsilon}\left(t+\epsilon^{2} s, t, x\right)+\epsilon y\right)$ and $\diamond=\left(t+\epsilon^{2} s, X_{n, \epsilon}\left(t+\epsilon^{2} s, t, x\right)\right)$, respectively. Then the chain rule gives us

$$
\begin{aligned}
& \partial_{s} v(s, y)=\epsilon^{3} \partial_{t}\left(u_{n}\right)(\square)+\epsilon^{3}\left(\left(u_{n} * \phi_{\frac{1}{n}} * \phi_{\epsilon}\right)(\diamond) \cdot \nabla\right) u_{n}(\square)-\epsilon \partial_{s}\left[\left(u_{n} * \phi_{\epsilon}\right)(\diamond)\right], \\
& \left(v * y \phi_{\frac{1}{n \epsilon}}\right)(s, y)=\epsilon\left(u_{n} * \phi_{\frac{1}{n}}\right)(\square)-\epsilon\left(u_{n} * \phi_{\epsilon}\right)(\diamond), \\
& \int_{\mathbb{R}^{3}}\left(v * y \phi_{\frac{1}{n \epsilon}}\right)(s, z) \phi(z) d z=\epsilon\left(u_{n} * \phi_{\frac{1}{n}} * \phi_{\epsilon}\right)(\diamond)-\epsilon\left(u_{n} * \phi_{\epsilon}\right)(\diamond), \\
& \left(\left(\left(v *_{y} \phi_{\frac{1}{n \epsilon}}\right)(s, y)-\int_{\mathbb{R}^{3}}\left(v *_{y} \phi_{\frac{1}{n \epsilon}}\right)(s, z) \phi(z) d z\right) \cdot \nabla\right) v(s, y) \\
& =\epsilon^{3}\left(\left(u_{n} * \phi_{\frac{1}{n}}\right)(\square) \cdot \nabla\right) u_{n}(\square)-\epsilon^{3}\left(\left(u_{n} * \phi_{\frac{1}{n}} * \phi_{\epsilon}\right)(\diamond) \cdot \nabla\right) u_{n}(\square), \\
& -\Delta_{y} v(s, y)=-\epsilon^{3} \Delta_{y} u_{n}(\square) \quad \text { and } \\
& \nabla_{y} Q(s, y)=\epsilon^{3} \nabla P_{n}(\square)+\epsilon \partial_{s}\left[\left(u_{n} * \phi_{\epsilon}\right)(\diamond)\right] .
\end{aligned}
$$

Thus, for $(s, y) \in(-4, \infty) \times \mathbb{R}^{3}$, we get

$$
\left[\partial_{s} v+\left(\left(\left(v * \phi_{\frac{1}{n \epsilon}}\right)-\int\left(v * \phi_{\frac{1}{n \epsilon}}\right) \phi\right) \cdot \nabla\right) v+\nabla Q-\Delta v\right](s, y)=0 .
$$

As a result, $\left(v\left(\cdot \cdot_{s},{ }_{y}\right), Q\left(\cdot{ }_{s}, \cdot_{y}\right)\right)$ is a solution of (Problem II- $\left.\frac{1}{n \epsilon}\right)$.

From the definition of the Maximal function, we can verify that $|\mathcal{M}(\nabla v)|^{2}$ has the right scaling as $|\nabla v|^{2}$ has in the following sense:

$$
\begin{aligned}
\mathcal{M}(\nabla v)(s, y) & =\sup _{M>0} \frac{C}{M^{3}} \int_{B(M)} \epsilon^{2}\left(\nabla u_{n}\right)\left(t+\epsilon^{2} s, X_{n, \epsilon}\left(t+\epsilon^{2} s, t, x\right)+\epsilon(y+z)\right) d z \\
& =\sup _{\epsilon M>0} \frac{C}{\epsilon^{3} M^{3}} \int_{B(\epsilon M)} \epsilon^{2}\left(\nabla u_{n}\right)\left(t+\epsilon^{2} s, X_{n, \epsilon}\left(t+\epsilon^{2} s, t, x\right)+\epsilon y+\bar{z}\right) d \bar{z} \\
& =\epsilon^{2} \mathcal{M}\left(\nabla u_{n}\right)(\square) .
\end{aligned}
$$

As a result, we have

$$
\begin{aligned}
& \int_{-4}^{0} \int_{B(2)}\left(|\nabla v(s, y)|^{2}+\left|\nabla^{2} Q(s, y)\right|+|\mathcal{M}(\nabla v)(s, y)|^{2}\right) d y d s \\
& =\epsilon^{4} \int_{-4}^{0} \int_{B(2)}\left[\left|\nabla u_{n}\right|^{2}+\left|\nabla^{2} P_{n}\right|+\left|\mathcal{M}\left(\nabla u_{n}\right)\right|^{2}\right](\square) d y d s \\
& =\epsilon^{4} \int_{-4}^{0} \int_{B(2)} F_{n}(\square) d y d s
\end{aligned}
$$




$$
=\epsilon^{-1} \int_{t-4 \epsilon^{2}}^{t} \int_{B(2 \epsilon)} F_{n}\left(s, X_{n, \epsilon}(s, t, x)+y\right) d y d s \leqslant \bar{\eta}
$$

where the first equality comes from the definition of $(v, Q)$, and the second one follows the change of variable $\left(t+\epsilon^{2} s, \epsilon y\right) \rightarrow(s, y)$. Moreover, it satisfies

$$
\int_{\mathbb{R}^{3}} \phi(z) v(s, z) d z=0, \quad-4<s<0 .
$$

So $(v, Q)$ satisfies all conditions of (21) and (22) in Proposition 2.2 with $r=1 /(n \epsilon) \in[0, \infty)$.

The conclusion of Proposition 2.2 implies that if $x \in \Omega_{n, \epsilon, t}$ for some $n, t$ and $\epsilon$ such that $4 \epsilon^{2} \leqslant t$ then $\left|\nabla^{d} v(0,0)\right| \leqslant C_{d}$. As a result, by using $\nabla^{d} v(0,0)=\epsilon^{d+1} \nabla^{d} u_{n}(t, x)$ for any integer $d \geqslant 1$, we have

$$
\left|\left\{x \in \mathbb{R}^{3}|| \nabla^{d} u_{n}(t, x) \mid>\frac{C_{d}}{\epsilon^{d+1}}\right\}\right| \leqslant\left|\Omega_{n, \epsilon, t}^{C}\right| \leqslant \epsilon^{4} \cdot \tilde{g}_{n}(t)
$$

Let $K$ be any open bounded subset in $\mathbb{R}^{3}$. Also we define $p=4 /(d+1)$. Then for any $t>0$, we have

$$
\beta^{p} \cdot\left|\left\{x \in K:\left|\left(\nabla^{d} u_{n}\right)(t, x)\right|>\beta\right\}\right| \leqslant \begin{cases}\beta^{p} \cdot|K|, & \text { if } \beta \leqslant C \cdot t^{-2 / p}, \\ C \cdot \tilde{g}_{n}(t), & \text { if } \beta>C \cdot t^{-2 / p} .\end{cases}
$$

It implies

$$
\left\|\left(\nabla^{d} u_{n}\right)(t, \cdot)\right\|_{L^{p, \infty}(K)}^{p} \leqslant C \cdot \max \left(\tilde{g}_{n}(t), \frac{|K|}{t^{2}}\right) .
$$

We pick any $t_{0}>0$. If we take $L^{1, \infty}\left(t_{0}, T\right)$-norm to the both sides of the above inequality, then we obtain

$$
\begin{aligned}
\left\|\nabla^{d} u_{n}\right\|_{L^{p, \infty}\left(t_{0}, \infty ; L^{p, \infty}(K)\right)}^{p} & \leqslant C\left(\left\|\tilde{g}_{n}\right\|_{L^{1, \infty}(0, \infty)}+|K| \cdot\left\|\frac{1}{|\cdot|^{2}}\right\|_{L^{1, \infty}\left(t_{0}, \infty\right)}\right) \\
& \leqslant C\left(\left\|u_{0}\right\|_{L^{2}\left(\mathbb{R}^{3}\right)}^{2}+\frac{|K|}{t_{0}}\right)
\end{aligned}
$$

where $C$ depends only on $d \geqslant 1$.

We observe that the above estimate is uniform in $n$. It is well known that both $\nabla u$ and $\nabla^{2} u$ are locally integrable functions if $u$ is a suitable weak solution $u$ which can be obtained by a limiting argument of $u_{n}$ (e.g. see Lions [29]). Thus, the above estimate (100) holds even for $u$ with $d=1,2$.

Remark 5.5. In fact, for the case $d=1$, the above estimate says $\nabla u \in L_{l o c}^{2, \infty}$, which is useless because we know a better estimate $\nabla u \in L^{2}$.

Remark 5.6. For $d \geqslant 3$, the above estimate (100) does not give us any direct information about higher derivatives $\nabla^{d} u$ of a weak solution $u$ because full regularity of weak solutions is still open, so $\nabla^{d} u$ may not be locally integrable for $d \geqslant 3$. Instead, the only thing we can say is that, for $d \geqslant 3$, higher derivatives $\nabla^{d} u_{n}$ of Leray's approximation $u_{n}$ have $L_{l o c}^{4 /(d+1), \infty}$ bounds which are uniform in $n \geqslant 1$.

From now on, we will prove the $0<\alpha<2$ case of the part (II).

\subsection{Proof of Theorem 1.1, part (II) for the $0<\alpha<2$ case}

Proof of Theorem 1.1, part (II) for the $0<\boldsymbol{\alpha}<\mathbf{2}$ case. We fix $d \geqslant 1$ and $0<\alpha<2$. Then, for any positive integer $n$, any $t>0$ and $x \in \mathbb{R}^{3}$, we denote $F_{n}(t, x)$ by: 


$$
\begin{aligned}
F_{n}(t, x)= & \left(\left|\nabla u_{n}(t, x)\right|^{2}+\left|\nabla^{2} P_{n}(t, x)\right|+\left|\mathcal{M}\left(\nabla u_{n}\right)(t, x)\right|^{2}+\left|\mathcal{M}\left(\mathcal{M}\left(\left|\nabla u_{n}\right|\right)\right)\right|^{2}+\left(\mathcal{M}\left(\left|\mathcal{M}\left(\left|\nabla u_{n}\right|\right)\right|^{q}\right)\right)^{2 / q}\right. \\
& \left.+\left|\mathcal{M}\left(\left|\nabla u_{n}\right|^{q}\right)\right|^{2 / q}+\sum_{m=d}^{d+4} \sup _{\delta>0}\left(\left|\left(\nabla^{m-1} h^{\alpha}\right)_{\delta} * \nabla^{2} P\right|\right)\right) .
\end{aligned}
$$

We define $g_{n}, \tilde{g}_{n}, X_{n, \epsilon}$ and $\Omega_{n, \epsilon, t}$ in a similar way as we did in the previous Section 5.2. Note that we have $\left\|\tilde{g}_{n}\right\|_{L^{1, \infty}(0, \infty)} \leqslant \frac{C_{d, \alpha}}{\bar{\eta}} \cdot\left\|u_{0}\right\|_{L^{2}\left(\mathbb{R}^{3}\right)}^{2}$ from Lemma 5.1.

Now we pick any $x \in \Omega_{n, \epsilon, t}$ and any $\epsilon$ such that $4 \epsilon^{2} \leqslant t$, and define $v$ and $Q$ as in the previous Section 5.2 (see (96)).

In order to follow the same way we did in the previous Section 5.2, we need to verify if all quantities in $F_{n}(t, x)$ have the right scaling after the transform (96). For Maximal of Maximal functions,

$$
\begin{aligned}
\mathcal{M}(\mathcal{M}(|\nabla v|))(s, y) & =\sup _{M>0} \frac{C}{M^{3}} \int_{B(M)} \mathcal{M}(|\nabla v|)(s, y+z) d z \\
& =\sup _{M>0} \frac{C}{M^{3}} \int_{B(M)} \epsilon^{2} \mathcal{M}\left(\left|\nabla u_{n}\right|\right)\left(t+\epsilon^{2} s, X_{n, \epsilon}\left(t+\epsilon^{2} s, t, x\right)+\epsilon(y+z)\right) d z \\
& =\epsilon^{2} \mathcal{M}\left(\mathcal{M}\left(\left|\nabla u_{n}\right|\right)\right)(\square)
\end{aligned}
$$

where $\square=\left(t+\epsilon^{2} s, X_{n, \epsilon}\left(t+\epsilon^{2} s, t, x\right)+\epsilon y\right)$ and we used the idea of (98) for the second and third equalities. Likewise, we get $\mathcal{M}\left(|\nabla v|^{q}\right)(s, y)=\epsilon^{2 q} \cdot \mathcal{M}\left(\left|\nabla u_{n}\right|^{q}\right)(\square)$ and $\mathcal{M}\left(|\mathcal{M}(|\nabla v|)|^{q}\right)(s, y)=\epsilon^{2 q} \cdot \mathcal{M}\left(\left|\mathcal{M}\left(\left|\nabla u_{n}\right|\right)\right|^{q}\right)(\square)$.

Also, we have, for any function $\mathcal{G} \in C_{0}^{\infty}$,

$$
\begin{aligned}
\sup _{\delta>0}\left(\left|\mathcal{G}_{\delta} * \nabla^{2} Q\right|\right)(s, y) & =\sup _{\delta>0}\left|\int_{\mathbb{R}^{3}} \frac{1}{\delta^{3}} \mathcal{G}\left(\frac{z}{\delta}\right) \cdot\left(\nabla^{2} Q\right)(s, y-z) d z\right| \\
& =\sup _{\delta>0}\left|\int_{\mathbb{R}^{3}} \frac{\epsilon^{4}}{\delta^{3}} \mathcal{G}\left(\frac{z}{\delta}\right) \cdot\left(\nabla^{2} P_{n}\right)\left(t+\epsilon^{2} s, X_{n, \epsilon}\left(t+\epsilon^{2} s, t, x\right)+\epsilon(y-z)\right) d z\right| \\
& =\sup _{\delta>0}\left|\int_{\mathbb{R}^{3}} \frac{\epsilon^{4}}{\epsilon^{3} \delta^{3}} \mathcal{G}\left(\frac{z}{\epsilon \delta}\right) \cdot\left(\nabla^{2} P_{n}\right)\left(t+\epsilon^{2} s, X_{n, \epsilon}\left(t+\epsilon^{2} s, t, x\right)+\epsilon y-z\right) d z\right| \\
& =\sup _{\epsilon \delta>0}\left|\int_{\mathbb{R}^{3}} \epsilon^{4} \mathcal{G}_{\epsilon \delta}(z) \cdot\left(\nabla^{2} P_{n}\right)\left(t+\epsilon^{2} s, X_{n, \epsilon}\left(t+\epsilon^{2} s, t, x\right)+\epsilon y-z\right) d z\right| \\
& =\sup _{\epsilon \delta>0} \epsilon^{4}\left|\left(\mathcal{G}_{\epsilon \delta} *\left(\nabla^{2} P_{n}\right)\right)\left(t+\epsilon^{2} s, X_{n, \epsilon}\left(t+\epsilon^{2} s, t, x\right)+\epsilon y\right)\right| \\
& =\epsilon^{4} \sup _{\delta>0}\left|\mathcal{G}_{\delta} *\left(\nabla^{2} P_{n}\right)\right|(\square) .
\end{aligned}
$$

Thus, by taking $\mathcal{G}=\left(\nabla^{m-1} h^{\alpha}\right)$, we have

$$
\sup _{\delta>0}\left(\left|\left(\nabla^{m-1} h^{\alpha}\right)_{\delta} * \nabla^{2} Q\right|\right)(s, y)=\epsilon^{4} \sup _{\delta>0}\left|\left(\nabla^{m-1} h^{\alpha}\right)_{\delta} *\left(\nabla^{2} P_{n}\right)\right|(\square) .
$$

As a result, we have

$$
\begin{aligned}
& \int_{-4}^{0} \int_{B(2)}\left[|\nabla v|^{2}+\left|\nabla^{2} Q\right|+|\mathcal{M}(\nabla v)|^{2}+|\mathcal{M}(\mathcal{M}(|\nabla v|))|^{2}+\left|\mathcal{M}\left(|\mathcal{M}(|\nabla v|)|^{q}\right)\right|^{q / 2}\right. \\
& \left.\quad+\left|\mathcal{M}\left(|\nabla v|^{q}\right)\right|^{2 / q}+\sum_{m=d}^{d+4} \sup _{\delta>0}\left(\left|\left(\nabla^{m-1} h^{\alpha}\right)_{\delta} * \nabla^{2} Q\right|\right)\right](s, y) d y d s
\end{aligned}
$$




$$
\begin{aligned}
& =\epsilon^{4} \int_{-4}^{0} \int_{B(2)} F_{n}(\square) d y d s \\
& =\epsilon^{-1} \int_{t-4 \epsilon^{2}}^{t} \int_{B(2 \epsilon)} F_{n}\left(s, X_{n, \epsilon}(s, t, x)+y\right) d y d s \leqslant \bar{\eta} .
\end{aligned}
$$

Then $(v, Q)$ satisfies (23) as well as (21) and (22) of Proposition 2.2 with $r=1 /(n \epsilon) \in[0, \infty)$. In sum if $x \in \Omega_{n, \epsilon, t}$ and if $4 \epsilon^{2} \leqslant t$, then we get

$$
\left|(-\Delta)^{\alpha / 2} \nabla^{d} v(0,0)\right| \leqslant C_{d, \alpha} .
$$

Because $u_{n}$ is a smooth solution of (Problem I- $n$ ), the fractional derivative $(-\Delta)^{\alpha / 2} \nabla^{d} u_{n}$ is not only a distribution but also a locally integrable function. Indeed, from a bootstrapping argument, it is easy to show that $\nabla^{d} u_{n}(t)$ has a desirable behavior at infinity which is required in order to use the integral representation (14) pointwise. For example, $\left(C^{2} \cap W^{2, \infty}\right)$ is enough (for a better approach, see Silvestre [37]). Also it can be easily verified that the resulting function $(-\Delta)^{\alpha / 2}\left[\nabla^{d} u_{n}(t, \cdot)\right](x)$ from the integral representation (14) satisfies the definition in Remark 1.1.

As a result, it makes sense to talk about pointwise values of $(-\Delta)^{\alpha / 2} \nabla^{d} u_{n}$. Note that, for any integer $d \geqslant 1$ and any real $0<\alpha<2$,

$$
(-\Delta)^{\alpha / 2} \nabla^{d} v(0,0)=\epsilon^{d+\alpha+1}(-\Delta)^{\alpha / 2} \nabla^{d} u_{n}(t, x) .
$$

Thus we can deduce the following set inclusion:

$$
\left\{x \in \mathbb{R}^{3}||(-\Delta)^{\frac{\alpha}{2}} \nabla^{d} u_{n}(t, x) \mid>\frac{C_{d, \alpha}}{\epsilon^{d+\alpha+1}}\right\} \subset \Omega_{n, \epsilon, t}^{C} .
$$

It implies, for any $0<t<\infty$ and for any $0<4 \epsilon^{2} \leqslant t$,

$$
\left|\left\{x \in \mathbb{R}^{3}||(-\Delta)^{\frac{\alpha}{2}} \nabla^{d} u_{n}(t, x) \mid>\frac{C_{d, \alpha}}{\epsilon^{d+\alpha+1}}\right\}\right| \leqslant\left|\Omega_{n, \epsilon, t}^{C}\right| \leqslant \epsilon^{4} \cdot \tilde{g}_{n}(t) .
$$

We define $p=4 /(d+\alpha+1)$. As we did for case $\alpha=0$, we obtain

$$
\left\|(-\Delta)^{\frac{\alpha}{2}} \nabla^{d} u_{n}\right\|_{L^{p, \infty}\left(t_{0}, \infty ; L^{p, \infty}(K)\right)}^{p} \leqslant C\left(\left\|u_{0}\right\|_{L^{2}\left(\mathbb{R}^{3}\right)}^{2}+\frac{|K|}{t_{0}}\right)
$$

for any integer $n, d \geqslant 1$, for any real $\alpha \in(0,2)$, for any bounded open subset $K$ of $\mathbb{R}^{3}$, and for any $t_{0} \in(0, \infty)$ where $C$ depends only on $d$ and $\alpha$.

If we restrict further $(d+\alpha)<3$, then $p=\frac{4}{d+\alpha+1}>1$. This implies $(-\Delta)^{\alpha / 2} \nabla^{d} u_{n} \in L_{l o c}^{q}\left(\left(t_{0}, \infty\right) \times K\right)$ for every $q$ between 1 and $p$, and the norm is uniformly bounded in $n$. Thus, from the weak-compactness of $L^{q}$ for $q>1$, we conclude that if $u$ is a suitable weak solution obtained by a limiting argument of $u_{n}$, then any higher derivative $(-\Delta)^{\alpha / 2} \nabla^{d} u$, which is defined by following Remark 1.1 , lie in $L_{l o c}^{1}$ as long as $(d+\alpha)<3$ with the same estimate

$$
\left\|(-\Delta)^{\frac{\alpha}{2}} \nabla^{d} u\right\|_{L^{p, \infty}\left(t_{0}, \infty ; L^{p, \infty}(K)\right)}^{p} \leqslant C_{d, \alpha}\left(\left\|u_{0}\right\|_{L^{2}\left(\mathbb{R}^{3}\right)}^{2}+\frac{|K|}{t_{0}}\right) .
$$

\subsection{Proof of Theorem 1.1, part (I)}

Proof of Theorem 1.1, part (I). Suppose that $(u, P)$ is a smooth solution of the Navier-Stokes equations (1) on $(0, T)$ with (2). Then it satisfies all conditions of (Problem I- $n$ ) for $n=\infty$ on $(0, T)$. As we mentioned in Remark 5.3, we follow every steps in Section 5.2 and 5.3 except each last arguments which require $d<3$ or $(d+\alpha)<3$. Indeed, under the scaling (96), the resulting function $(v, Q)$ is a solution for (Problem II- $r$ ) for $r=0$.

Recall that $u$ is smooth by assumption. As a result, we do NOT have any restriction like $d<3$ or $(d+\alpha)<3$ at this time because we do not need any limiting argument any more which requires the weak-compactness of $L^{q}$ for $q>1$. Thus, we obtain (102) for any integer $d \geqslant 1$, for any real $\alpha \in[0,2)$ and for any $t_{0} \in(0, T)$. It finishes the proof of the part (I) of the main Theorem 1.1. 


\section{Acknowledgements}

The second author was partially supported by both the NSF and the EPSRC Science and Innovation award to the Oxford Centre for Nonlinear PDE (EP/E035027/1).

\section{Appendix A. Proofs of some technical lemmas}

Proof of Lemma 4.1. We fix $(n, a, b, p)$ such that $n \geqslant 2,0<b<a<1$ and $1<p<\infty$. Let $\alpha$ be any multi index such that $|\alpha|=n$ and $D^{\alpha}=\partial_{\alpha_{1}} \partial_{\alpha_{2}} D^{\beta}$ where $\beta$ is a multi index with $|\beta|=n-2$.

Observe that, from $\operatorname{div}\left(v_{2}\right)=0$ and $\operatorname{div}\left(v_{1}\right)=0$, we have

$$
\begin{aligned}
-\Delta\left(D^{\alpha} P\right) & =\operatorname{div} \operatorname{div} D^{\alpha}\left(v_{2} \otimes v_{1}\right) \\
& =D^{\alpha}\left(\sum_{i j}\left(\partial_{j} v_{2, i}\right)\left(\partial_{i} v_{1, j}\right)\right) \\
& =\partial_{\alpha_{1}} \partial_{\alpha_{2}} H
\end{aligned}
$$

where $H=D^{\beta}\left(\sum_{i j}\left(\partial_{j} v_{2, i}\right)\left(\partial_{i} v_{1, j}\right)\right)$ and $v_{k}=\left(v_{k, 1}, v_{k, 2}, v_{k, 3}\right)$ for $k=1,2$. Then, for any $\left(p_{1}, p_{2}\right)$ such that $\frac{1}{p}=$ $\frac{1}{p_{1}}+\frac{1}{p_{2}}$, we get

$$
\|H\|_{L^{p}(B(a))} \leqslant C\left\|v_{2}\right\|_{W^{n-1, p_{2}(B(a))}} \cdot\left\|v_{1}\right\|_{W^{n-1, p_{1}(B(a))}}
$$

where $C$ is independent of choice of $p_{1}$ and $p_{2}$ and

$$
\|H\|_{W^{1, \infty}(B(a))} \leqslant C\left\|v_{2}\right\|_{W^{n, \infty}(B(a))} \cdot\left\|v_{1}\right\|_{W^{n, \infty}(B(a))} .
$$

We fix a function $\psi \in C^{\infty}\left(\mathbb{R}^{3}\right)$ satisfying:

$$
\psi=1 \quad \text { in } B\left(b+\frac{a-b}{3}\right), \quad \psi=0 \quad \text { in }\left(B\left(b+\frac{2(a-b)}{3}\right)\right)^{C} \text { and } 0 \leqslant \psi \leqslant 1 .
$$

We decompose $D^{\alpha} P$ by using $\psi$ :

$$
\begin{aligned}
-\Delta\left(\psi D^{\alpha} P\right) & =-\psi \Delta D^{\alpha} P-2 \operatorname{div}\left((\nabla \psi)\left(D^{\alpha} P\right)\right)+\left(D^{\alpha} P\right) \Delta \psi \\
& =\psi \partial_{\alpha_{1}} \partial_{\alpha_{2}} H-2 \operatorname{div}\left((\nabla \psi)\left(D^{\alpha} P\right)\right)+\left(D^{\alpha} P\right) \Delta \psi \\
& =-\Delta Q_{1}-\Delta Q_{2}-\Delta Q_{3}
\end{aligned}
$$

where

$$
\begin{aligned}
& -\Delta Q_{1}=\partial_{\alpha_{1}} \partial_{\alpha_{2}}(\psi H), \\
& -\Delta Q_{2}=-\partial_{\alpha_{2}}\left[\left(\partial_{\alpha_{1}} \psi\right)(H)\right]-\partial_{\alpha_{1}}\left[\left(\partial_{\alpha_{2}} \psi\right)(H)\right]+\left(\partial_{\alpha_{1}} \partial_{\alpha_{2}} \psi\right)(H) \quad \text { and } \\
& -\Delta Q_{3}=-2 \operatorname{div}\left((\nabla \psi)\left(D^{\alpha} P\right)\right)+\left(D^{\alpha} P\right) \Delta \psi .
\end{aligned}
$$

Here $Q_{2}$ and $Q_{3}$ are defined by the representation formula $(-\Delta)^{-1}(f)=\frac{1}{4 \pi}\left(\frac{1}{|x|} * f\right)$ while $Q_{1}$ is defined by the Riesz transforms.

Then, by the Riesz transform, we get

$$
\begin{aligned}
\left\|Q_{1}\right\|_{L^{p}(B(b))} & \leqslant C\|\psi H\|_{L^{p}\left(\mathbb{R}^{3}\right)} \leqslant C\|H\|_{L^{p}(B(a))} \\
& \leqslant C\left\|v_{2}\right\|_{W^{n-1, p_{2}(B(a))}} \cdot\left\|v_{1}\right\|_{W^{n-1, p_{1}(B(a))}} .
\end{aligned}
$$

Moreover, using Sobolev's inequality, we have

$$
\begin{aligned}
\left\|Q_{1}\right\|_{L^{\infty}(B(b))} & \leqslant C\left(\left\|Q_{1}\right\|_{L^{4}(B(b))}+\left\|\nabla Q_{1}\right\|_{L^{4}(B(b))}\right) \\
& \leqslant C\|H\|_{W^{1,4}(B(a))} \leqslant C\|H\|_{W^{1, \infty}(B(a))} \\
& \leqslant C\left\|v_{2}\right\|_{W^{n, \infty}(B(a))} \cdot\left\|v_{1}\right\|_{W^{n, \infty}(B(a)) .}
\end{aligned}
$$


For $x \in B(b)$, we compute

$$
\begin{aligned}
\left|Q_{2}(x)\right|= & \mid \frac{1}{4 \pi} \int_{\left(B\left(b+\frac{2(a-b)}{3}\right)-B\left(b+\frac{a-b}{3}\right)\right)} \frac{1}{|x-y|}\left(\partial_{\alpha_{2}}\left[\left(\partial_{\alpha_{1}} \psi\right)(H)\right](y)\right. \\
& \left.-\partial_{\alpha_{1}}\left[\left(\partial_{\alpha_{2}} \psi\right)(H)\right](y)+\left(\partial_{\alpha_{1}} \partial_{\alpha_{2}} \psi\right)(H)(y)\right) d y \mid \\
\leqslant & 2\|\nabla \psi\|_{L^{\infty}} \cdot \sup _{y \in B\left(b+\frac{a-b}{3}\right) C}\left(\left|\nabla_{y} \frac{1}{|x-y|}\right|\right) \cdot\|H\|_{L^{1}(B(a))} \\
& +\left\|\nabla^{2} \psi\right\|_{L^{\infty}} \cdot \sup _{y \in B\left(b+\frac{a-b}{3}\right) C}\left(\left|\frac{1}{|x-y|}\right|\right) \cdot\|H\|_{L^{1}(B(a))} \\
\leqslant & C \cdot\|H\|_{L^{1}(B(a))}
\end{aligned}
$$

because $|x-y| \geqslant(a-b) / 3$. Likewise, for $x \in B(b)$, we get

$$
\begin{aligned}
\left|Q_{3}(x)\right| \leqslant & C\left(\sum_{k=0}^{n}\left\|\nabla^{k+1} \psi\right\|_{L^{\infty}}\right) \cdot\left(\sum_{k=0}^{n} \sup _{y \in B\left(b+\frac{a-b}{3}\right)^{C}}\left|\nabla_{y}^{k+1} \frac{1}{|x-y|}\right|\right) \cdot\|P\|_{L^{1}(B(a))} \\
& +C\left(\sum_{k=0}^{n}\left\|\nabla^{k+2} \psi\right\|_{L^{\infty}}\right) \cdot\left(\sum_{k=0}^{n} \sup _{y \in B\left(b+\frac{a-b}{3}\right)}\left|\nabla_{y}^{k} \frac{1}{|x-y|}\right|\right) \cdot\|P\|_{L^{1}(B(a))} \\
\leqslant & C \cdot\|P\|_{L^{1}(B(a))} .
\end{aligned}
$$

Finally, we obtain

$$
\begin{aligned}
\left\|\nabla^{n} P\right\|_{L^{p}(B(b))} & \leqslant\left\|Q_{1}\right\|_{L^{p}(B(b))}+C\left\|\left|Q_{2}\right|+\left|Q_{3}\right|\right\|_{L^{\infty}(B(b))} \\
& \leqslant C \cdot\|H\|_{L^{p}(B(a))}+C \cdot\|H\|_{L^{1}(B(a))}+C \cdot\|P\|_{L^{1}(B(a))} \\
& \leqslant C_{a, b, p, n}\left(\left\|v_{2}\right\|_{W^{n-1, p_{2}(B(a))}} \cdot\left\|v_{1}\right\|_{W^{n-1, p_{1}(B(a))}}+\|P\|_{L^{1}(B(a))}\right)
\end{aligned}
$$

and

$$
\begin{aligned}
\left\|\nabla^{n} P\right\|_{L^{\infty}(B(b))} & \leqslant\left\|\left|Q_{1}\right|+\left|Q_{2}\right|+\left|Q_{3}\right|\right\|_{L^{\infty}(B(b))} \\
& \leqslant C \cdot\|H\|_{W^{1, \infty}(B(a))}+C \cdot\|H\|_{L^{1}(B(a))}+C \cdot\|P\|_{L^{1}(B(a))} \\
& \leqslant C_{a, b, n}\left(\left\|v_{2}\right\|_{W^{n, \infty}(B(a))} \cdot\left\|v_{1}\right\|_{W^{n, \infty}(B(a))}+\|P\|_{L^{1}(B(a))}\right) .
\end{aligned}
$$

Proof of Lemma 4.3. We fix $(n, a, b)$ such that $n \geqslant 0$ and $0<b<a<1$ and let $\alpha$ be a multi index with $|\alpha|=n$. Then, by taking $D^{\alpha}$ to (18), we have

$$
0=\partial_{t}\left(D^{\alpha} v_{1}\right)+\sum_{\beta \leqslant \alpha,|\beta|>0}\left(\begin{array}{l}
\alpha \\
\beta
\end{array}\right)\left(\left(D^{\beta} v_{2}\right) \cdot \nabla\right)\left(D^{\alpha-\beta} v_{1}\right)+\left(v_{2} \cdot \nabla\right)\left(D^{\alpha} v_{1}\right)+\nabla\left(D^{\alpha} P\right)-\Delta\left(D^{\alpha} v_{1}\right) .
$$

We define $\Phi(t, x) \in C^{\infty}$ by $0 \leqslant \Phi \leqslant 1, \Phi=1$ on $Q_{b}$ and $\Phi=0$ on $Q_{a}^{C}$. We observe that, for $p \geqslant \frac{1}{2}$ and for $f \in C^{\infty}$,

$$
\left(p+\frac{1}{2}\right)|f|^{p-\frac{3}{2}} f \cdot \partial_{x} f=\partial_{x}|f|^{p+\frac{1}{2}} \quad \text { and } \quad\left(p+\frac{1}{2}\right)|f|^{p-\frac{3}{2}} f \cdot \Delta f \leqslant \Delta\left(|f|^{p+\frac{1}{2}}\right)
$$

which can be verified directly due to the fact $|\nabla f| \geqslant|\nabla| f||$.

Now we multiply $\left(p+\frac{1}{2}\right) \Phi \frac{D^{\alpha} v_{1}}{\left|D^{\alpha} v_{1}\right|^{(3 / 2)-p}}$ to (103), use the above observation and integrate in $x$ to get: for any $p \geqslant \frac{1}{2}$,

$$
\frac{d}{d t} \int_{\mathbb{R}^{3}} \Phi(t, x)\left|D^{\alpha} v_{1}(t, x)\right|^{p+\frac{1}{2}} d x
$$




$$
\begin{aligned}
& \leqslant \int_{\mathbb{R}^{3}}\left(\left|\partial_{t} \Phi(t, x)\right|+|\Delta \Phi(t, x)|\right)\left|D^{\alpha} v_{1}(t, x)\right|^{p+\frac{1}{2}} d x \\
& +\left(p+\frac{1}{2}\right) \int_{\mathbb{R}^{3}}\left|\nabla D^{\alpha} P(t, x)\right|\left|D^{\alpha} v_{1}(t, x)\right|^{p-\frac{1}{2}} d x \\
& +\left(p+\frac{1}{2}\right) \sum_{\beta \leqslant \alpha,|\beta|>0}\left(\begin{array}{c}
\alpha \\
\beta
\end{array}\right) \int_{\mathbb{R}^{3}}\left|\left(D^{\beta} v_{2}(t, x) \cdot \nabla\right) D^{\alpha-\beta} v_{1}(t, x)\right|\left|D^{\alpha} v_{1}(t, x)\right|^{p-\frac{1}{2}} d x \\
& -\int_{\mathbb{R}^{3}} \Phi(t, x)\left(v_{2}(t, x) \cdot \nabla\right)\left(\left|D^{\alpha} v_{1}(t, x)\right|^{p+\frac{1}{2}}\right) d x \\
& \leqslant C\left\|\left|\nabla^{n} v_{1}(t, \cdot)\right|^{p+\frac{1}{2}}\right\|_{L^{1}(B(a))} \\
& +C\left\|\nabla^{n+1} P(t, \cdot)\right\|_{L^{2 p}(B(a))} \cdot\left\|\left|\nabla^{n} v_{1}(t, \cdot)\right|^{p-\frac{1}{2}}\right\|_{L^{\frac{2 p}{2 p-1}(B(a))}} \\
& +C\left\|v_{2}(t, \cdot)\right\|_{W^{n, \infty}(B(a))} \cdot\left\|v_{1}(t, \cdot)\right\|_{W^{n, p+\frac{1}{2}(B(a))}} \cdot\left\|\left|\nabla^{n} v_{1}(t, \cdot)\right|^{p-\frac{1}{2}}\right\|_{L^{\frac{p+\frac{1}{2}}{p-\frac{1}{2}}(B(a))}} \\
& -\int_{\mathbb{R}^{3}} \Phi(t, x) \operatorname{div}\left(v_{2}(t, x) \otimes\left|D^{\alpha} v_{1}(t, x)\right|^{p+\frac{1}{2}}\right) d x \\
& \leqslant C\left\|v_{1}(t, \cdot)\right\|_{W^{n, p+\frac{1}{2}}(B(a))}^{p+\frac{1}{2}} \\
& +C\left\|\nabla^{n+1} P(t, \cdot)\right\|_{L^{2 p}(B(a))} \cdot\left\|\nabla^{n} v_{1}(t, \cdot)\right\|_{L^{p}(B(a))}^{p-\frac{1}{2}} \\
& +C\left\|v_{2}(t, \cdot)\right\|_{W^{n, \infty}(B(a))} \cdot\left\|v_{1}(t, \cdot)\right\|_{W^{n, p+\frac{1}{2}(B(a))}}^{p+\frac{1}{2}} \\
& +C\left\|v_{2}(t, \cdot)\right\|_{L^{\infty}(B(a))} \cdot\left\|\nabla^{n} v_{1}(t, \cdot)\right\|_{L^{p+\frac{1}{2}(B(a))}}^{p+\frac{1}{2}} .
\end{aligned}
$$

Then the integration on $\left[-a^{2}, t\right]$ for any $t \in\left[-b^{2}, 0\right]$ gives us

$$
\begin{aligned}
& \left\|D^{\alpha} v_{1}\right\|_{L^{\infty}\left(-(b)^{2}, 0 ; L^{p+\frac{1}{2}}(B(b))\right)}^{p+\frac{1}{2}} \\
& \leqslant C\left\|v_{1}\right\|_{L^{p+\frac{1}{2}}\left(-(a)^{2}, 0 ; W^{n, p+\frac{1}{2}}(B(a))\right)}^{p+\frac{1}{2}} \\
& +C\left\|\nabla^{n+1} P\right\|_{L^{1}\left(-(a)^{2}, 0 ; L^{2 p}(B(a))\right)} \cdot\left\|\nabla^{n} v_{1}\right\|_{L^{\infty}\left(-(a)^{2}, 0 ; L^{p}(B(a))\right)}^{p-\frac{1}{2}} \\
& +C\left\|v_{2}\right\|_{L^{2}\left(-(a)^{2}, 0 ; W^{n, \infty}(B(a))\right)} \cdot\left\|v_{1}\right\|_{L^{2 p+1}\left(-(a)^{2}, 0 ; W^{n, p+\frac{1}{2}}(B(a))\right)}^{p+\frac{1}{2}} \\
& +C\left\|v_{2}\right\|_{L^{2}\left(-(a)^{2}, 0 ; L^{\infty}(B(a))\right)} \cdot\left\|\nabla^{n} v_{1}\right\|_{L^{2 p+1}\left(-(a)^{2}, 0 ; L^{\left.p+\frac{1}{2}(B(a))\right)}\right.}^{p+\frac{1}{2}} .
\end{aligned}
$$

Thus for the case $p=1 / 2$, we have

$$
\begin{aligned}
& \left\|D^{\alpha} v_{1}\right\|_{L^{\infty}\left(-(b)^{2}, 0 ; L^{1}(B(b))\right)} \\
& \quad \leqslant C\left[\left(\left\|v_{2}\right\|_{L^{2}\left(-a^{2}, 0 ; W^{n, \infty}(B(a))\right)}+1\right) \cdot\left\|v_{1}\right\|_{L^{2}\left(-a^{2}, 0 ; W^{n, 1}(B(a))\right)}+\left\|\nabla^{n+1} P\right\|_{L^{1}\left(-a^{2}, 0 ; L^{1}(B(a))\right)}\right]
\end{aligned}
$$

while, for the case $p \geqslant 1$, we have 


$$
\begin{aligned}
& \left\|D^{\alpha} v_{1}\right\|_{L^{\infty}\left(-(b)^{2}, 0 ; L^{\left.p+\frac{1}{2}(B(b))\right)}\right.}^{p+\frac{1}{2}} \\
& \leqslant C\left(\left\|v_{2}\right\|_{L^{2}\left(-a^{2}, 0 ; W^{n, \infty}(B(a))\right)}+1\right)\left(\left\|v_{1}\right\|_{L^{2}\left(-(a)^{2}, 0 ; W^{n, 2 p}(B(a))\right)}^{\frac{1}{p+\frac{1}{2}}} \cdot\left\|v_{1}\right\|_{L^{\infty}\left(-(a)^{2}, 0 ; W^{n, p}(B(a))\right)}^{1-\frac{1}{p+\frac{1}{2}}}\right)^{p+\frac{1}{2}} \\
& \quad+C\left\|\nabla^{n+1} P\right\|_{L^{1}\left(-(a)^{2}, 0 ; L^{2 p}(B(a))\right)} \cdot\left\|v_{1}\right\|_{L^{\infty}\left(-\frac{1}{2}\right.}^{\left.p(a)^{2}, 0 ; W^{n, p}(B(a))\right)} \\
& \leqslant C_{a, b, n, p}\left[\left(\left\|v_{2}\right\|_{L^{2}\left(-a^{2}, 0 ; W^{n, \infty}(B(a))\right)}+1\right) \cdot\left\|v_{1}\right\|_{L^{2}\left(-(a)^{2}, 0 ; W^{n, 2 p}(B(a))\right)}\right. \\
& \left.\quad+\left\|\nabla^{n+1} P\right\|_{L^{1}\left(-(a)^{2}, 0 ; L^{2 p}(B(a))\right)}\right] \cdot\left\|v_{1}\right\|_{L^{\infty}\left(-(a)^{2}, 0 ; W^{n, p}(B(a))\right)}^{p-\frac{1}{2}}
\end{aligned}
$$

Proof of Lemma 4.4. Let $M_{0}>0$ and $1 \leqslant p<\infty$. Then, for any $M \geqslant M_{0}$ and for any $f \in C^{1}\left(\mathbb{R}^{3}\right)$ such that $\int_{\mathbb{R}^{3}} \phi(x) f(x) d x=0$, we have

$$
\begin{aligned}
\|f\|_{L^{p}(B(M))} & \left(\int_{B(M)}\left|\int_{\mathbb{R}^{3}}(f(x)-f(y)) \phi(y) d y\right|^{p} d x\right)^{1 / p} \\
\leqslant & C\left(\int_{B(M)}\left(\int_{B(1)}|f(x)-f(y)| d y\right)^{p} d x\right)^{1 / p} \\
\leqslant & C\left(\int_{B(M)}\left(\int_{B(1)} \int_{0}^{1}|(\nabla f)((1-t) x+t y) \cdot(x-y)| d t d y\right)^{p} d x\right)^{1 / p} \\
\leqslant & C(M+1)\left(\int_{B(M)}\left(\int_{B(1)} \int_{0}^{1}|(\nabla f)((1-t) x+t y)| d t d y\right)^{p} d x\right)^{1 / p} \\
\leqslant & C(M+1)\left(\int_{B(M)}\left(\int_{B(1)} \int_{0}^{\frac{M}{M+1}}|(\nabla f)((1-t) x+t y)| d t d y\right)^{p} d x\right)^{1 / p} \\
& +C(M+1)\left(\int_{B(M)}\left(\int_{B(1)} \int_{M}^{M}|(\nabla f)((1-t) x+t y)| d t d y\right)^{p} d x\right)^{1 / p} \\
= & (I)+(I I)
\end{aligned}
$$

where we used $x \in B(M)$ and $y \in B(1)$.

For $(I)$, we have

$$
\begin{aligned}
(I) & \leqslant C_{M_{0}} \cdot M \cdot\left(\int_{B(1)} \int_{0}^{\frac{M}{M+1}}\left(\int_{B(M)}|(\nabla f)((1-t) x+t y)|^{p} d x\right)^{1 / p} d t d y\right) \\
& \leqslant C_{M_{0}} \cdot M \cdot \int_{0}^{\frac{M}{M+1}} \frac{1}{(1-t)^{3 / p}}\left(\int_{B((1-t) M+1)}|(\nabla f)(z)|^{p} d z\right)^{1 / p} d t \\
& \leqslant C_{M_{0}} \cdot M \cdot \int_{0}^{\frac{M}{M+1}} \frac{1}{(1-t)^{3 / p}}\left(\int_{B(1)} \int_{B((1-t) M+2)}|(\nabla f)(z+u)|^{p} d z d u\right)^{1 / p} d t
\end{aligned}
$$




$$
\begin{aligned}
& \leqslant C_{M_{0}} \cdot M \cdot \int_{0}^{\frac{M}{M+1}} \frac{((1-t) M+2)^{3 / p}}{(1-t)^{3 / p}}\left(\int_{B(1)} \mathcal{M}\left(|\nabla f|^{p}\right)(u) d u\right)^{1 / p} d t \\
& \leqslant C_{M_{0}, p} \cdot M \cdot\left\|\mathcal{M}\left(|\nabla f|^{p}\right)\right\|_{L^{1}(B(1))}^{1 / p} \int_{0}^{\frac{M}{M+1}}\left(M^{3 / p}+\frac{1}{(1-t)^{3 / p}}\right) d t \\
& \leqslant C_{M_{0}, p} \cdot M \cdot\left\|\mathcal{M}\left(|\nabla f|^{p}\right)\right\|_{L^{1}(B(1))}^{1 / p}\left(M^{3 / p}+\int_{\frac{1}{M+1}}^{1} \frac{1}{s^{3 / p}} d s\right) \\
& \leqslant C_{M_{0}} \cdot M \cdot\left\|\mathcal{M}\left(|\nabla f|^{p}\right)\right\|_{L^{1}(B(1))}^{1 / p}\left(M^{3 / p}+(M+1)^{3 / p}\right) \\
& \leqslant C_{M_{0}, p} \cdot M M^{1+\frac{3}{p}} \cdot\left\|\mathcal{M}\left(|\nabla f|^{p}\right)\right\|_{L^{1}(B(1))}^{1 / p}
\end{aligned}
$$

where we used the integral version of Minkowski's inequality and the fact $(1+M) \leqslant C_{M_{0}} \cdot M$ from $M \geqslant M_{0}$ for the first inequality.

For $(I I)$, we observe that if $\frac{M}{M+1} \leqslant t \leqslant 1$, then $0 \leqslant 1-t \leqslant \frac{1}{M+1}$ and

$$
|(1-t) x+t y| \leqslant(1-t) \cdot|x|+t|y| \leqslant \frac{M}{M+1}+1 \leqslant 2
$$

due to $x \in B(M)$ and $y \in B(1)$. Thus, we have

$$
\begin{aligned}
(I I) & \leqslant C_{M_{0}} \cdot M\left(\int_{B(M)}\left(\int_{\frac{M}{M+1}}^{1} \frac{1}{t^{3}} \int_{B(2)}|(\nabla f)(z)| d z d t\right)^{p} d x\right)^{1 / p} \\
& \leqslant C_{M_{0}} \cdot M \cdot M^{3 / p} \cdot \int_{B(2)}|(\nabla f)(z)| d z \cdot \int_{\frac{M}{M+1}}^{1} \frac{1}{t^{3}} d t \\
& \leqslant C_{M_{0}} M^{1+\frac{3}{p}} \cdot\|\nabla f\|_{L^{1}(B(2))} \cdot
\end{aligned}
$$

\section{References}

[1] J.T. Beale, T. Kato, A. Majda, Remarks on the breakdown of smooth solutions for the 3-D Euler equations, Commun. Math. Phys. 94 (1) (1984) 61-66.

[2] H. Beirão da Veiga, A new regularity class for the Navier-Stokes equations in $\mathbf{R}^{n}$, Chin. Ann. Math., Ser. B 16 (4) (1995) 407-412; a Chinese summary appears in Chin. Ann. Math., Ser. A 16 (6) (1995) 797.

[3] Jöran Bergh, Jörgen Löfström, Interpolation Spaces. An Introduction, Grundlehren Math. Wiss., vol. 223, Springer-Verlag, Berlin, 1976.

[4] Luigi C. Berselli, Giovanni P. Galdi, Regularity criteria involving the pressure for the weak solutions to the Navier-Stokes equations, Proc. Am. Math. Soc. 130 (12) (2002) 3585-3595 (electronic).

[5] Clayton Bjorland, Alexis Vasseur, Weak in space, log in time improvement of the Ladyženskaja-Prodi-Serrin criteria, J. Math. Fluid Mech. 13 (2) (2011) 259-269.

[6] L. Caffarelli, R. Kohn, L. Nirenberg, Partial regularity of suitable weak solutions of the Navier-Stokes equations, Commun. Pure Appl. Math. 35 (6) (1982) 771-831.

[7] Luis A. Caffarelli, Alexis Vasseur, Drift diffusion equations with fractional diffusion and the quasi-geostrophic equation, Ann. Math. 171 (3) (2010) 1903-1930.

[8] Dongho Chae, Kyungkeun Kang, Jihoon Lee, On the interior regularity of suitable weak solutions to the Navier-Stokes equations, Commun. Partial Differ. Equ. 32 (7-9) (2007) 1189-1207.

[9] Chi Hin Chan, Smoothness criterion for Navier-Stokes equations in terms of regularity along the streamlines, Methods Appl. Anal. 17 (1) (2010) 81-103.

[10] A. Cheskidov, R. Shvydkoy, The regularity of weak solutions of the 3D Navier-Stokes equations in $B_{\infty, \infty}^{-1}$, Arch. Ration. Mech. Anal. 195 (2010) 159-169.

[11] R. Coifman, P.-L. Lions, Y. Meyer, S. Semmes, Compensated compactness and Hardy spaces, J. Math. Pures Appl. 72 (3) (1993) $247-286$. 
[12] Peter Constantin, Navier-Stokes equations and area of interfaces, Commun. Math. Phys. 129 (2) (1990) 241-266.

[13] Ennio De Giorgi, Sulla differenziabilità e l'analiticità delle estremali degli integrali multipli regolari, Mem. Accad. Sci. Torino, Cl. Sci. Fis. Mat. Nat. 3 (1957) 25-43.

[14] Hongjie Dong, Dapeng Du, On the local smoothness of solutions of the Navier-Stokes equations, J. Math. Fluid Mech. 9 (2) (2007) 139-152.

[15] G.F.D. Duff, Derivative estimates for the Navier-Stokes equations in a three-dimensional region, Acta Math. 164 (3-4) (1990) $145-210$.

[16] L. Escauriaza, G.A. Seregin, V. Šverák, $L_{3, \infty}$-solutions of Navier-Stokes equations and backward uniqueness, Usp. Mat. Nauk 58 (2(350)) (2003) 3-44.

[17] C. Fefferman, E.M. Stein, $H^{p}$ spaces of several variables, Acta Math. 129 (3-4) (1972) 137-193.

[18] Ciprian Foiaş, Colette Guillopé, Roger Temam, New a priori estimates for Navier-Stokes equations in dimension 3, Commun. Partial Differ. Equ. 6 (3) (1981) 329-359.

[19] Yoshikazu Giga, Okihiro Sawada, On regularizing-decay rate estimates for solutions to the Navier-Stokes initial value problem, in: Nonlinear Analysis and Applications: To V. Lakshmikantham on His 80th birthday. vols. 1, 2, Kluwer Acad. Publ., Dordrecht, 2003 , pp. 549-562.

[20] Loukas Grafakos, Classical and Modern Fourier Analysis, Pearson Education, Inc., Upper Saddle River, NJ, 2004.

[21] Stephen Gustafson, Kyungkeun Kang, Tai-Peng Tsai, Interior regularity criteria for suitable weak solutions of the Navier-Stokes equations, Commun. Math. Phys. 273 (1) (2007) 161-176.

[22] Eberhard Hopf, Über die Anfangswertaufgabe für die hydrodynamischen Grundgleichungen, Math. Nachr. 4 (1951) $213-231$.

[23] Igor Kukavica, The fractal dimension of the singular set for solutions of the Navier-Stokes system, Nonlinearity 22 (12) (2009) $2889-2900$.

[24] O.A. Ladyženskaja, Uniqueness and smoothness of generalized solutions of Navier-Stokes equations, Zap. Naučn. Sem. Leningrad. Otdel. Mat. Inst. Steklov. (LOMI) 5 (1967) 169-185.

[25] O.A. Ladyženskaja, V.A. Solonnikov, N.N. Ural'ceva, Linear and Quasilinear Equations of Parabolic Type, Transl. Math. Monogr., vol. 23, American Mathematical Society, Providence, RI, 1967, translated from the Russian by S. Smith.

[26] P.G. Lemarié-Rieusset, Recent Developments in the Navier-Stokes Problem, Chapman \& Hall/CRC Res. Notes Math., vol. 431, Chapman \& Hall/CRC, Boca Raton, FL, 2002.

[27] Jean Leray, Sur le mouvement d'un liquide visqueux emplissant l'espace, Acta Math. 63 (1) (1934) 193-248.

[28] Fanghua Lin, A new proof of the Caffarelli-Kohn-Nirenberg theorem, Commun. Pure Appl. Math. 51 (3) (1998) $241-257$.

[29] Pierre-Louis Lions, Mathematical topics in fluid mechanics, vol. 1, in: Incompressible Models, in: Oxford Sci. Publ., Oxford Lecture Ser. Math. Appl., vol. 3, The Clarendon Press Oxford University Press, New York, 1996.

[30] Giovanni Prodi, Un teorema di unicità per le equazioni di Navier-Stokes, Ann. Mat. Pura Appl. (4) 48 (1959) $173-182$.

[31] James C. Robinson, Witold Sadowski, Decay of weak solutions and the singular set of the three-dimensional Navier-Stokes equations, Nonlinearity 20 (5) (2007) 1185-1191.

[32] Laurent Saloff-Coste, Aspects of Sobolev-Type Inequalities, Lond. Math. Soc. Lect. Note Ser., vol. 289, Cambridge University Press, Cambridge, 2002.

[33] Vladimir Scheffer, The Navier-Stokes equations on a bounded domain, Commun. Math. Phys. 73 (1) (1980) 1-42.

[34] Maria E. Schonbek, Michael Wiegner, On the decay of higher-order norms of the solutions of Navier-Stokes equations, Proc. R. Soc. Edinb., Sect. A 126 (3) (1996) 677-685.

[35] G.A. Seregin, A new version of the Ladyzhenskaya-Prodi-Serrin condition, Algebra Anal. 18 (1) (2006) 124-143.

[36] James Serrin, The initial value problem for the Navier-Stokes equations, in: Nonlinear Problems, Proc. Sympos., Madison, WI, Univ. of Wisconsin Press, Madison, WI, 1963, pp. 69-98.

[37] Luis Silvestre, Regularity of the obstacle problem for a fractional power of the Laplace operator, Commun. Pure Appl. Math. 60 (1) (2007) 67-112.

[38] V.A. Solonnikov, A priori estimates for solutions of second-order equations of parabolic type, Tr. Mat. Inst. Steklova 70 (1964) $133-212$.

[39] Elias M. Stein, Harmonic analysis: real-variable methods, orthogonality, and oscillatory integrals, in: Monographs in Harmonic Analysis, III, in: Princeton Math. Ser., vol. 43, Princeton University Press, Princeton, NJ, 1993, with the assistance of Timothy S. Murphy.

[40] Michael Struwe, On partial regularity results for the Navier-Stokes equations, Commun. Pure Appl. Math. 41 (4) (1988) $437-458$.

[41] Alexis Vasseur, Higher derivatives estimate for the 3D Navier-Stokes equation, Ann. Inst. Henri Poincaré, Anal. Non Linéaire 27 (5) (2010) 1189-1204.

[42] Alexis F. Vasseur, A new proof of partial regularity of solutions to Navier-Stokes equations, Nonlinear Differ. Equ. Appl. 14 (5-6) (2007) 753-785.

[43] Jörg Wolf, A direct proof of the Caffarelli-Kohn-Nirenberg theorem, in: Parabolic and Navier-Stokes Equations. Part 2, in: Banach Cent. Publ., vol. 81, Polish Acad. Sci. Inst. Math, Warsaw, 2008, pp. 533-552. 